\title{
Development of Laboratory Test Methods for RF-Based Electronic Safety Equipment: Guide to the National Fire Protection Association 1982 Standard
}

\author{
Audrey K. Puls \\ William F. Young \\ Kate A. Remley \\ Jacob Healy \\ Luis A. Gonzalez
}

This publication is available free of charge from:

https://doi.org/10.6028/NIST.TN.1937 


\title{
Development of Laboratory Test Methods for RF-Based Electronic Safety Equipment: Guide to the National Fire Protection Association 1982 Standard
}

\author{
Audrey K. Puls \\ William F. Young \\ Kate A. Remley \\ Jacob Healy \\ Luis A. Gonzalez \\ RF Technology Division \\ Communications Technology Laboratory
}

This publication is available free of charge from: https://doi.org/10.6028/NIST.TN.1937

October 2016

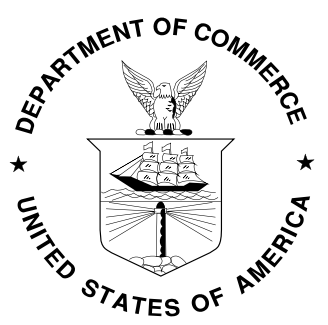

U.S. Department of Commerce Penny Pritzker, Secretary

National Institute of Standards and Technology Willie May, Under Secretary of Commerce for Standards and Technology and Director 
Certain commercial entities, equipment, or materials may be identified in this document in order to describe an experimental procedure or concept adequately. Such identification is not intended to imply recommendation or endorsement by the National Institute of Standards and Technology, nor is it intended to imply that the entities, materials, or equipment are necessarily the best available for the purpose.

National Institute of Standards and Technology Technical Note 1937

Natl. Inst. Stand. Technol. Tech. Note 1937, 74 pages (October 2016)

CODEN: NTNOEF

This publication is available free of charge from: https://doi.org/10.6028/NIST.TN.1937 


\section{Executive Summary}

The National Institute of Standards and Technology (NIST) with support from the Department of Homeland Security has been working with the National Fire Protection Association (NFPA) to develop standards and test methods for radio-frequency personal alert safety systems (RF PASS). The RF PASS systems are incorporated into a firefighter's gear and are used to alert a base station when the firefighter is in distress, has been motionless for more than 30 seconds, or to alert the firefighter in case an evacuation is necessary.

The material here covers the development of several test methods either already adopted by the NFPA or targeted for future revisions of NFPA 1982 Personal Alert Safety Systems. These test methods represent five RF PASS deployment conditions: a highly reflective (high multipath) RF environment, a prescribed amount of attenuation in a point-to-point communication link, a multihop connection path, in-band wireless activity, and temporary exposure to high-power, in-band RF interference. The data and developed testing are based on currently used RF PASS frequencies: $450 \mathrm{MHz}, 900 \mathrm{MHz}$, and $2.4 \mathrm{GHz}$.

This technical note uses various experimental setups that utilize a combination of equipment including, but not limited to, spectrum analyzers, signal generators, power combiners, anechoic chambers, and reverberation chambers. Specific experimental setups are discussed in the sections in which they apply.

In developing the test methods, several key aspects of the test setup are investigated in detail. For example, electric field uniformity within the test chambers, created by different antennas, was examined to determine which antenna would provide the lowest variability in signal level over the testing plane. In support of the multipath test, the RF reflective behavior of a reverberation chamber was evaluated. Finally, interference testing required establishing the duty cycles and power levels appropriate for anticipated deployment environments.

Supporting material from previous RF PASS in-field testing, along with correlated RF propagation measurements are used to set test parameter values. For example, the attenuation values used for the various links represent typical conditions measured in the field and reported in prior NIST work. Some in-field data are also used in establishing the interference duty cycles and power levels. The ultimate goal was to establish methods and parameter values that mimic real deployment conditions in a laboratory setting to the extent possible.

Finally, the intent of this document is to provide users of the test methods with insight into how the tests were developed and why certain procedures and values were adopted. This document is not intended to give step-by-step directions on how each test should be conducted. Rather, the material contained in this document should answer questions that may arise during the application of the tests. Thus, the aim is to capture the insight gained by the test developers in a manner that will allow others to obtain the same understanding in a shorter time period. 


\title{
Development of Laboratory Test Methods for RF- Based Electronic Safety Equipment: Guide to the National Fire Protection Association 1982 Standard
}

\author{
Audrey K. Puls, William F. Young, Kate A. Remley, Jacob Healy, and Luis A. Gonzalez \\ RF Technology Division \\ National Institute of Standards and Technology \\ 325 Broadway, Boulder, CO 80305
}

\begin{abstract}
We discuss a variety of standardized test methods that have been developed to assess radio-frequency personal alert safety systems (RF PASS) that are used as "firefighter down" beacons. The test methods found in this document represent five RF PASS deployment conditions: a highly reflective (high multipath) RF environment; a prescribed amount of attenuation in a pointto-point communication link; a multi-hop reception path; in-band wireless activity; and temporary exposure to high-power, in-band RF interference. The tests and corresponding data are based on current RF PASS frequencies: $450 \mathrm{MHz}, 900 \mathrm{MHz}$, and $2.4 \mathrm{GHz}$. The test environments, tests, and measured data are discussed in detail to accompany the National Fire Protection Association's standard "NFPA 1982: Personal Alert Safety Systems."
\end{abstract}

Key words: anechoic chamber; firefighter; interference; multipath; personal alert safety system; point-to-point radio communications; path loss; radio-wave propagation; reverberation chamber; wireless system. 


\section{Contents}

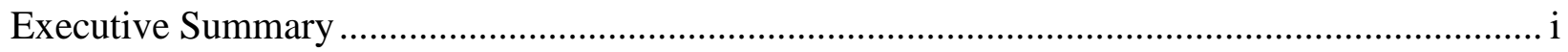

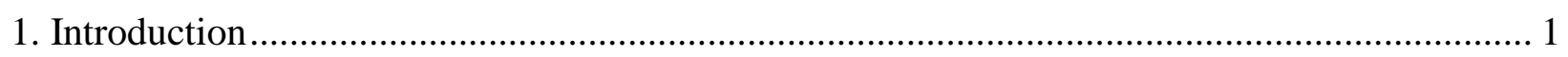

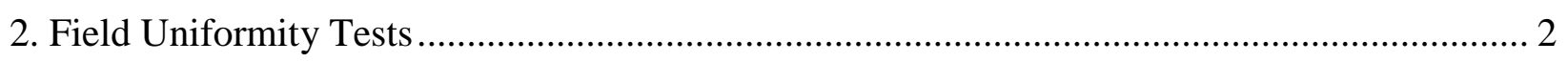

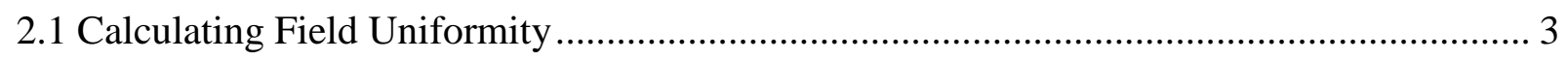

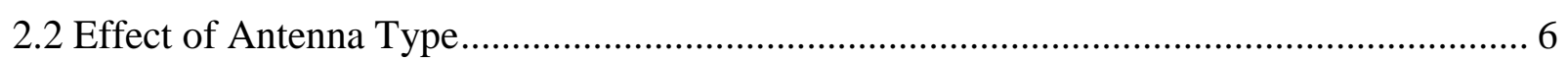

2.3 NIST Field-Uniformity Measurement Results.............................................................. 8

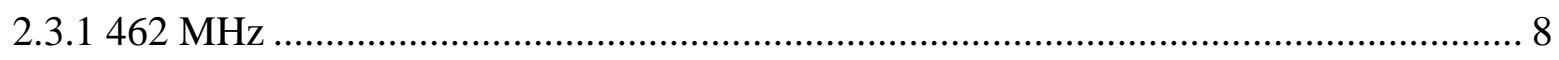

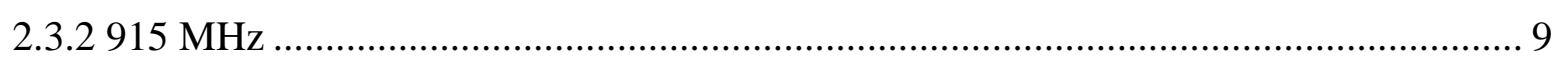

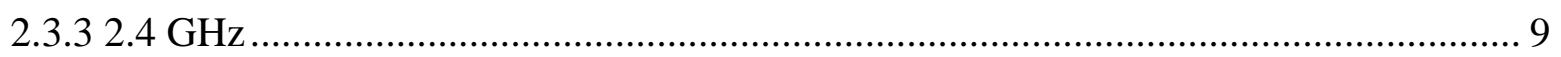

2.3.3.1 Uniformity Testing with 13 Collection Points...................................................... 10

2.3.3.2 Uniformity Testing with 25 Collection Points........................................................ 11

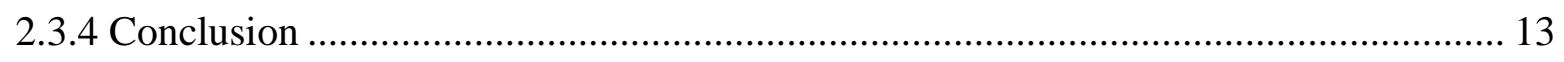

2.4 Field Uniformity Measurement Uncertainty Analysis.................................................... 14

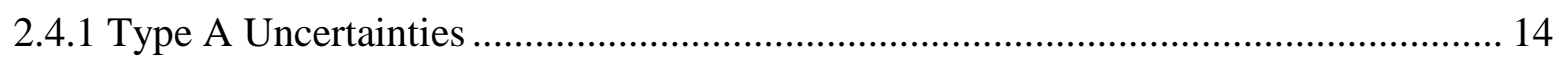

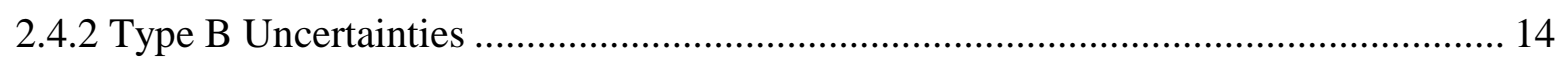

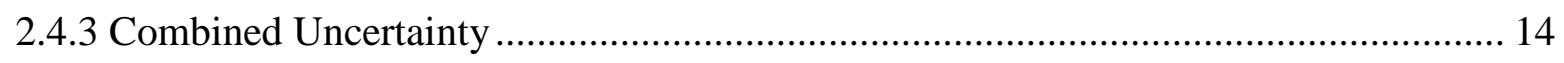

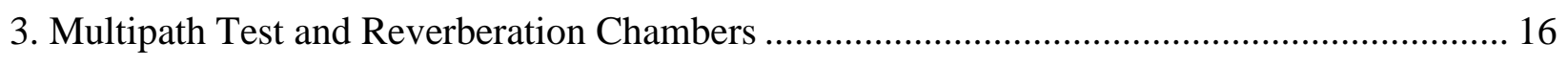

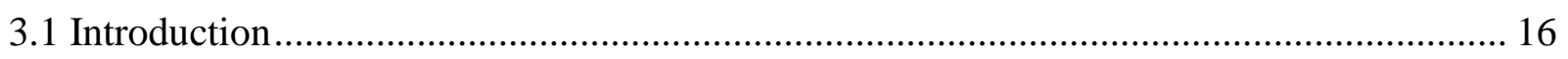

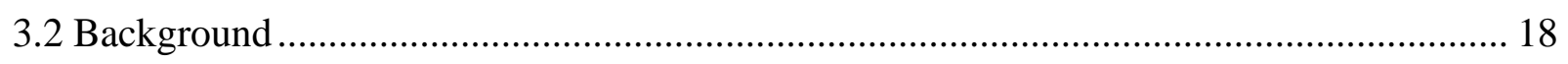

3.3 Quality of the Reverberation Chamber ..................................................................... 19

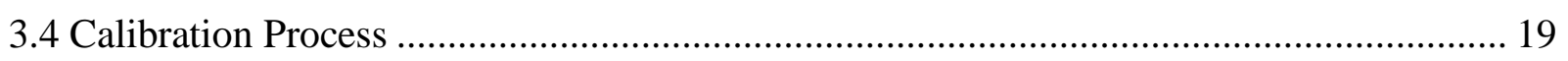

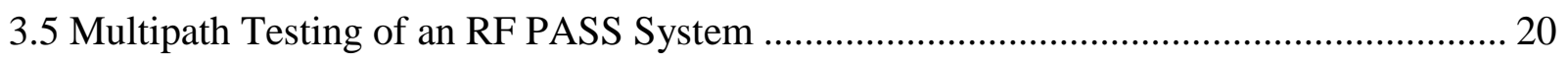

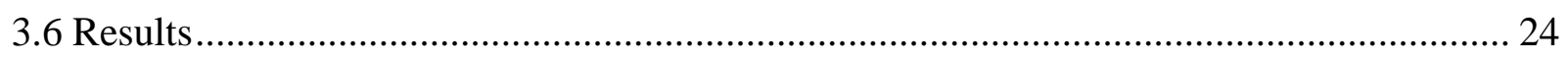

4. Configuration Considerations for Point-Point, Multi-Hop, and Interference Testing ............... 25

4.1 Path Loss Measurements for Anechoic Chamber ............................................................ 26

4.1.1 Measurement of Cable Loss for an Individual Anechoic Chamber Setup................... 27

4.1.2 Determining Antenna Type for RF PASS Testing Configuration ............................... 27

4.1.3 Path Loss between Two Anechoic Chambers............................................................ 29

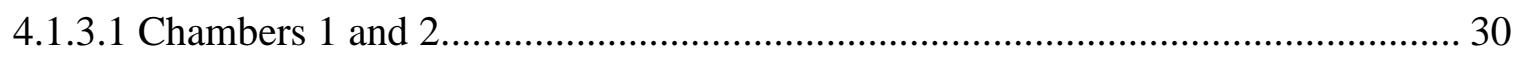

4.1.3.2 Chambers 1 and 2 with Added Attenuation...................................................... 31 
4.1.4 RF PASS Performance between Two Anechoic Chambers ..................................... 32

4.2. Reverberation Chamber Calibration and RF PASS Stirrer Speed Dependency ............... 32

4.2.1 Reverberation Chamber Calibration .................................................................... 32

4.2.2 Stirrer Speed Dependency 2.4 GHz Manufacturer 3 RF PASS .............................. 33

4.2.3 Stirrer Speed Dependency 900 MHz Manufacturer 1 RF PASS ............................... 34

4.2.4 Stirrer Speed Dependency 900 MHz Manufacturer 2 RF PASS ............................ 35

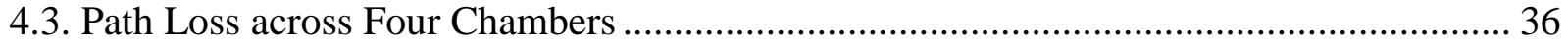

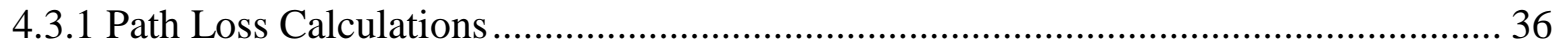

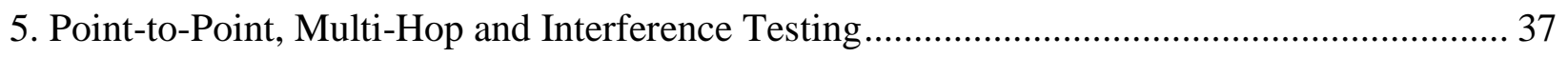

5.1 Point-to-Point Motion and Evacuation Alarm Test ................................................... 37

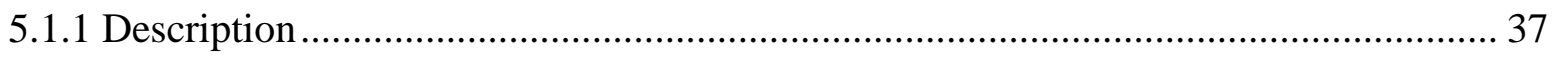

5.1.2 NIST Tests: Point-to-Point Motion Alarm Results................................................ 39

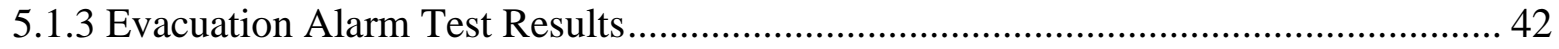

5.1.4 Point-to-Point Test Uncertainty Analysis .......................................................... 43

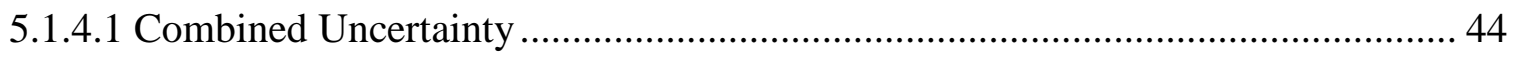

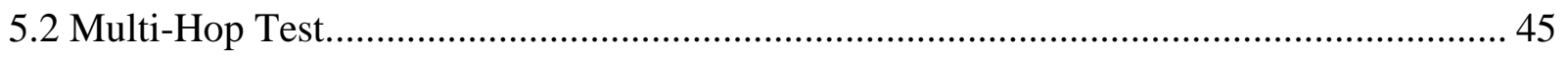

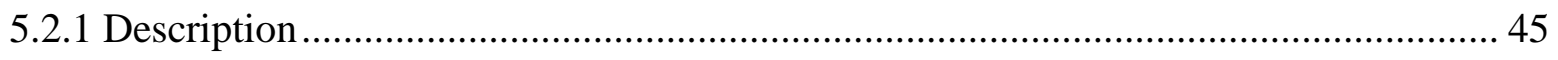

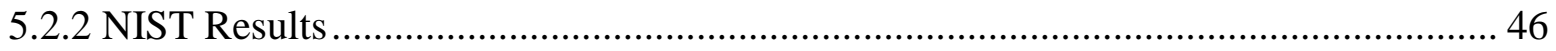

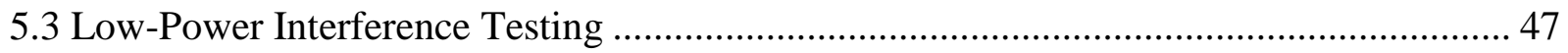

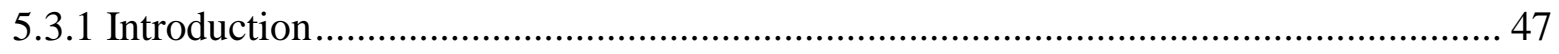

5.3.2 The Target Value of Interference ................................................................... 49

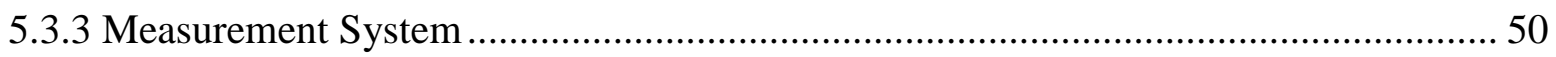

5.3.4 Specific Interference Test Configurations for $900 \mathrm{MHz}$ and $2.4 \mathrm{GHz}$ Systems......... 51

5.3.5 Measurement Procedure for Interference Testing............................................... 52

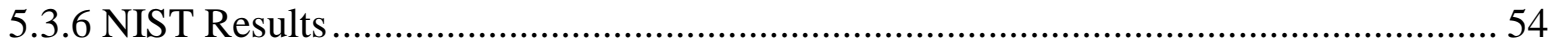

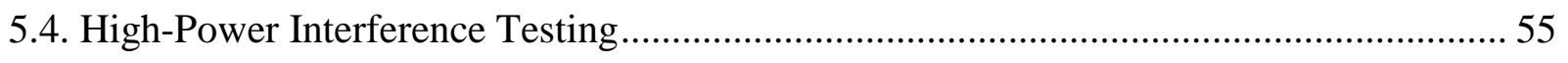

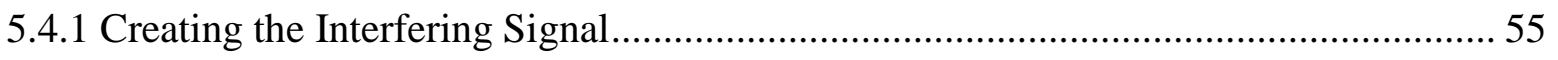

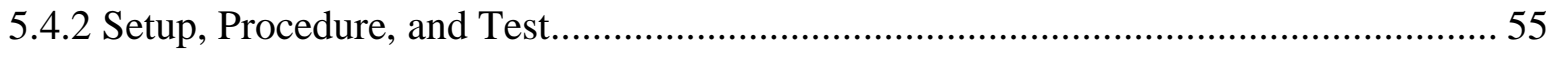

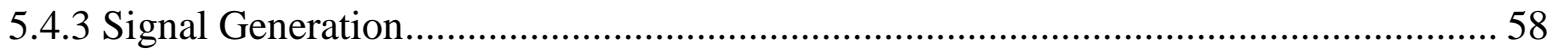

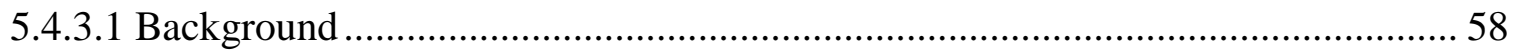

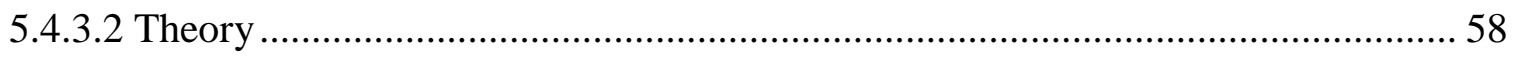

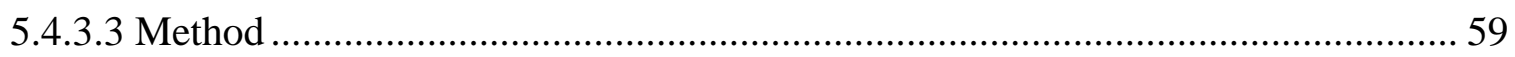




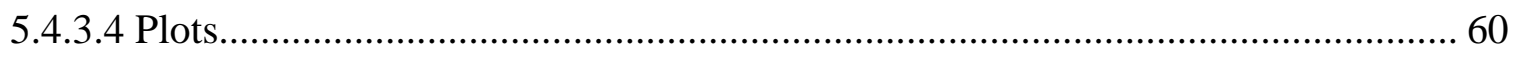

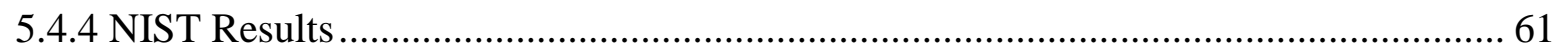

5.4.4.1 Manufacturer 3 High-Power Interference Test Results .................................. 61

5.4.4.2 Manufacturer 1 High-Power Interference Test Results .................................. 62

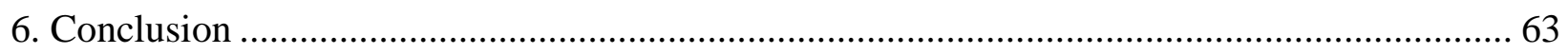




\section{Introduction}

Personal Alert Safety Systems (PASS) are used by firefighters to indicate an emergency "firefighter-down" condition. In traditional PASS, when a firefighter is motionless for 30 seconds, a loud, audible alarm is emitted from the body-worn PASS beacon, alerting firefighters nearby that another firefighter is in distress [1]. RF-based PASS is a relatively new product technology that can potentially extend the reach of the PASS beacon from the firefighter to the incident command station outside a structure. They also allow the incident commander to send an "evacuate" command to the firefighter inside a structure, a new and potentially life-saving technology.

For firefighting companies that choose to use RF-based PASS, test methods to assess performance under representative environmental conditions can increase confidence in the use of this technology. To this end, two NIST-developed test methods were incorporated into the 2013 revision of "NFPA 1982 Personal Alert Safety Systems" [1] to test the operation of RF PASS under typical point-to-point attenuation (path loss) conditions and in the presence of in-band RF interference (coexistence). These two test methods were also detailed in [2] and [3]. Additional test methods have recently been developed at NIST. One method tests the operation of RF-based PASS systems in highly reflective, "multipath" environments such as factories, refineries, and warehouses. A second test is designed to evaluate RF-based PASS performance in high-attenuation environments such as subways and large high-rise buildings. Work is underway to test RF-based PASS in the presence of strong interfering signals. Note that over-the-air performance tests (as opposed to cabled tests) are necessary because system-level verification is required and antennas may be integrated into the PASS or self-contained breathing apparatus. This complicates test procedures. The details on how these over-the-air test methods are implemented in a laboratory environment is the subject of this Technical Note.

From 2008 through 2016, the Department of Homeland Security funded researchers from the National Institute of Standards and Technology (NIST) to support the development of rigorous test methods for RF-based PASS systems. NIST researchers performed several field tests to characterize RF propagation channels in representative firefighting conditions where RF-based PASS communication was expected to be poor, focusing on point-to-point signal penetration into and out of medium-and large-sized structures. The researchers conducted side-by-side tests of the RF channel characteristics (path loss and reflectivity) and RF-based PASS in order to determine parameters and metrics for laboratory-based tests.

NIST Technical Note 1559 [3] summarizes NIST measurements made in seven environments: an urban canyon (downtown Denver, CO), a 12-story apartment building, three one-story office buildings, a high-rise office building, and a convention center. These studies summarized the level of path loss and multipath (given by the metric "root-mean-square (RMS) delay spread") between the incident command and portable RF PASS. This study showed a total path loss of approximately $100 \mathrm{~dB}$ for a point-to-point link. This study also reported measured values of RMS delay spread ranging from tens of ns to over $400 \mathrm{~ns}$. 
NIST Technical Note 1546 "Measurements to Support Modulated-Signal Radio Transmissions for the Public-Safety Sector" [5] describes measurements in which the level of multipath measured in three environments: an office corridor, an oil refinery, and a subterranean tunnel. Values of RMS delay spread ranged from tens to over $200 \mathrm{~ns}$.

NIST Technical Note 1792 details additional studies where RF PASS were deployed in a subway and in the Empire State Building in New York City. Here, path-loss values up to $260 \mathrm{~dB}$ were observed. The RF PASS systems that utilized repeaters were found to be able to penetrate deeper.

Based on the NIST measurement studies, parameter values used in the NFPA test methods were recommended as $100 \mathrm{~dB}$ for a point-to-point link, $\sim 260 \mathrm{~dB}$ for a multiple-hop system, and at least $400 \mathrm{~ns}$ for RMS delay spread. The anechoic/reverberation chamber set-ups in the test methods described below meet these requirements. Details on how the test chambers are configured to provide these parameter values are given below.

The development of these laboratory-based test methods for RF PASS required investigation into components of the test setup, parameter settings, and electromagnetic environments in order to ensure a robust, repeatable test with sufficiently low measurement uncertainty. Once those parameters were well-established, the actual test configurations were constructed.

In the next several sections, the concepts of field uniformity, path loss (or channel attenuation), multipath and interference are discussed. The information should help in understanding how these elements support the basic concepts of the test methods discussed in the later sections of this document.

Throughout this document various acronyms are used which are outlined in Table 1.

Table 1. Acronyms

\begin{tabular}{|c|c|}
\hline Acronym & Definition \\
\hline NIST & National Institute of Standards and Technology \\
\hline RF PASS & Radio-Frequency Personal Alert Safety System \\
\hline NFPA & National Fire Protection Association \\
\hline PXI & PCI eXtension (Used for Signal Generation) \\
\hline SA & Spectrum Analyzer \\
\hline SG & Signal Generator \\
\hline RC & Reverberation Chamber \\
\hline AC & Anechoic Chamber \\
\hline
\end{tabular}

\section{Field Uniformity Tests}

All of the RF PASS test methods in NFPA 1982 require the use of anechoic chambers. Ideally, the anechoic chamber configuration should provide the same electromagnetic (EM) or RF signal conditions to the portable unit and base station at a specified region within the chamber to support repeatability of the test results. A uniform field ${ }^{1}$ at a specific region within the anechoic chamber theoretically allows the RF PASS to be placed in any orientation at that location and experience

\footnotetext{
${ }^{1}$ Uniform field in this document is used to describe an isotropic field. An isotropic field is uniform in all orientations.
} 
the same or similar field conditions. In order to quantify the electric field uniformity, field strengths were determined at the specific frequencies of interest, namely $462 \mathrm{MHz}, 915 \mathrm{MHz}$, and $2.4 \mathrm{GHz}$. The frequencies listed here are in the nominal carrier frequencies or within communication bands of the RF PASS systems used in developing the test methods.

\subsection{Calculating Field Uniformity}

To verify the field uniformity across the test platform in each anechoic chamber, a three-axis field probe was used. The electric field probe collected measurements in volts/meter $(\mathrm{V} / \mathrm{m})$ which in post processing were normalized by the minimum value measured with (1). Figure 1 details the basic probe collection setup at the table surface in the anechoic chamber. A dielectric square table sits at the bottom of the anechoic chamber, as shown in Figure 1, and the two data measurement patterns on the surface of the table are shown in Figure 2 and Figure 3. Figure 4 illustrates the probe $\mathrm{x}-, \mathrm{y}-$, and z-orientation.

The normalized uniformity, reported in decibels $(\mathrm{dB})$ was based on the total field, i.e., the contribution from all three of the orthogonal components. If the variation in the total field across the middle $30 \mathrm{~cm}$ by $30 \mathrm{~cm}$ of the platform was less than $3 \mathrm{~dB}$, the field was considered uniform. Other standards use $6 \mathrm{~dB}$ as the threshold for uniformity measurements. However, due to the small platform size in this case a tighter standard of $3 \mathrm{~dB}$ is required. The normalized field at each point is given by equation (1).

Normalized Field (dB)

$$
=20 \times \log _{10}\left(\frac{\text { Specific Probe Value Averaged Over Three Components }\left(\frac{\mathrm{V}}{\mathrm{m}}\right)}{\text { Minimum Probe Value Across Entire Table }\left(\frac{\mathrm{V}}{\mathrm{m}}\right)}\right)
$$

Two different measurement patterns were used. The first pattern, shown in Figure 2, consisted of 13 measurement points [6]. The uniformity of the field was determined by interpolating between measured values and plotting the contour mapping of the resultant field. This measurement approach is used in the NFPA 1982, 2013 edition standard [1].

To adapt the field uniformity measurement process more closely to traditional EMC standards such as [6], a larger number of points were collected, with the option to ignore up to $25 \%$ of the points. Twenty-five measurement points are utilized in the pattern shown in Figure 3. In determining the field uniformity, $25 \%$ of the 25 points could be discarded.

The anechoic chamber used by NIST for this and other tests throughout this technical note is $187.96 \mathrm{~cm}$ (74 inches) high, $91.44 \mathrm{~cm}$ (36 inches) wide and long, and has $100 \mathrm{~dB}$ shielding. Chambers with different dimensions can be used so long as they are rated for the frequency of the device under test. 


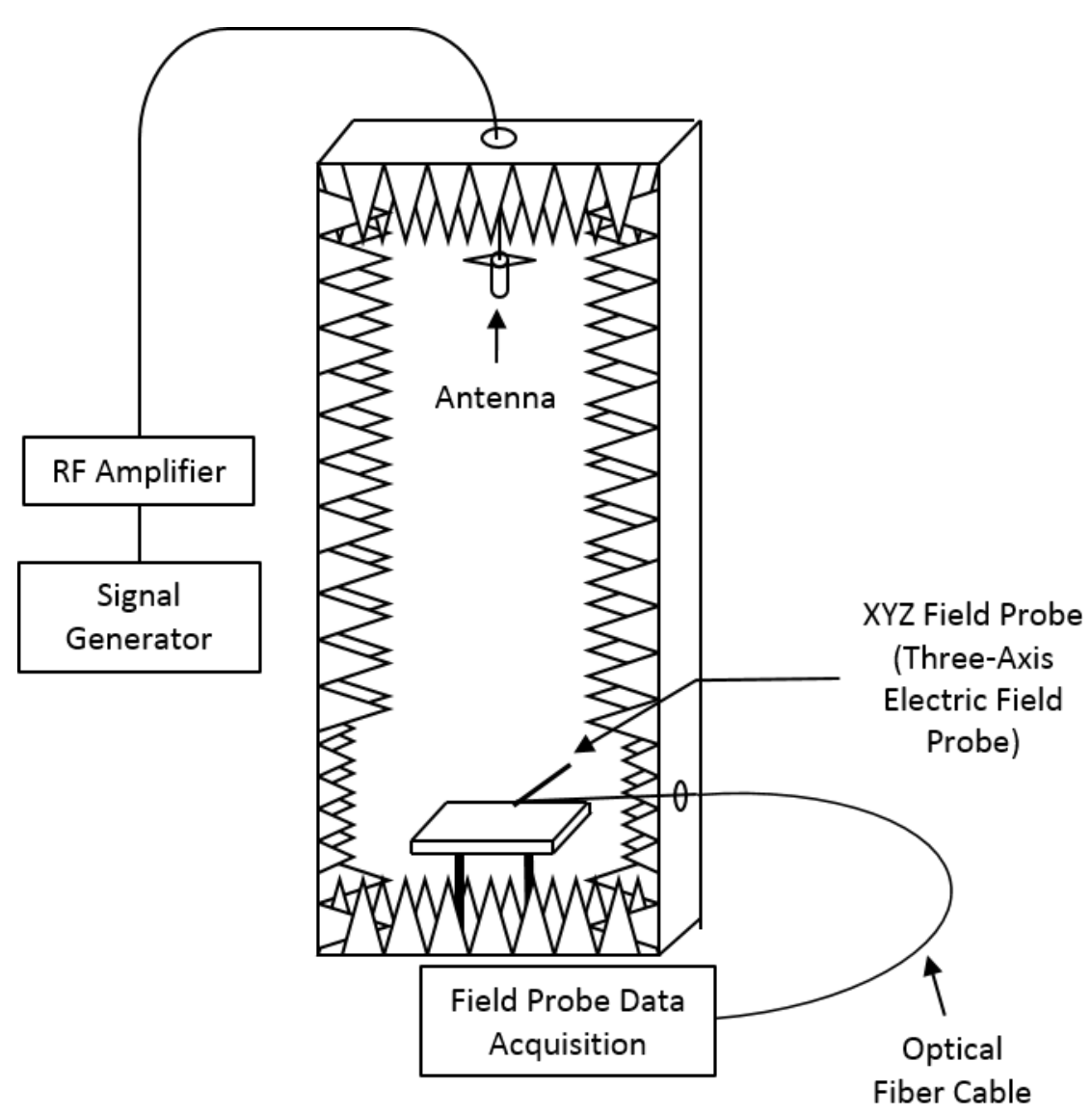

Figure 1. Setup for Field Uniformity Test.

A key feature of the setup in Figure 1 is the inclusion of the RF amplifier. This ensures that the signal read by the probe is sufficiently above the minimum sensitivity of the field probe. Also, Figure 1 depicts an anechoic chamber with a table area that is less than $0.5 \mathrm{~m}$ by $0.5 \mathrm{~m}$. The relative proximity of the sides of the chamber to some DUT's can make it difficult to achieve a uniform field at the measurement plane. The measurements and discussions below provide examples of how a lack of uniformity can be overcome. 


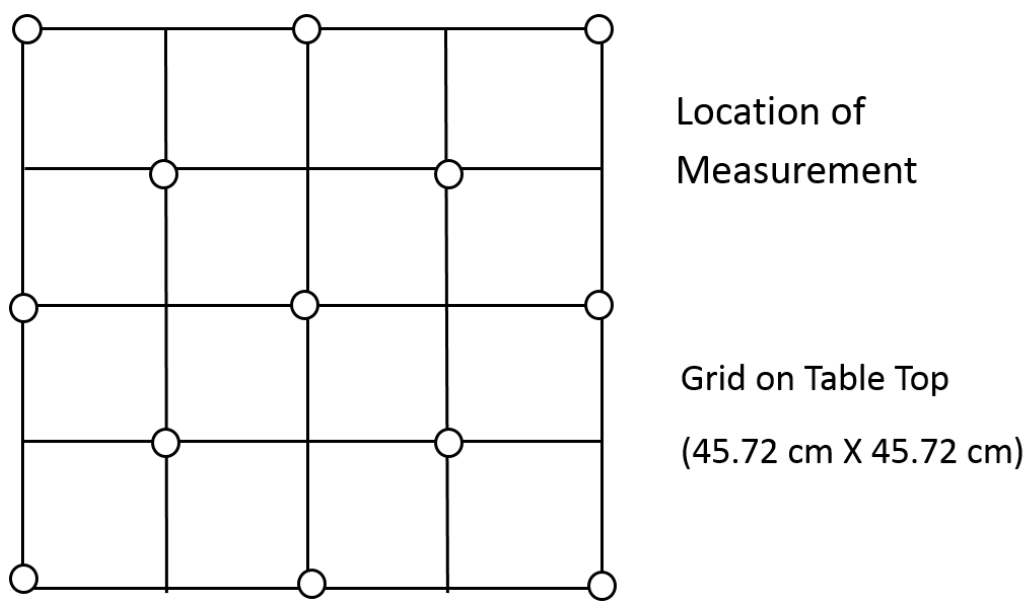

Figure 2.Table top placed within the anechoic chamber showing markings where a 3-axis field probe was placed. These 13 points were used for the $450 \mathrm{MHz}, 900 \mathrm{MHz}$, and $2.4 \mathrm{GHz}$ uniformity tests.

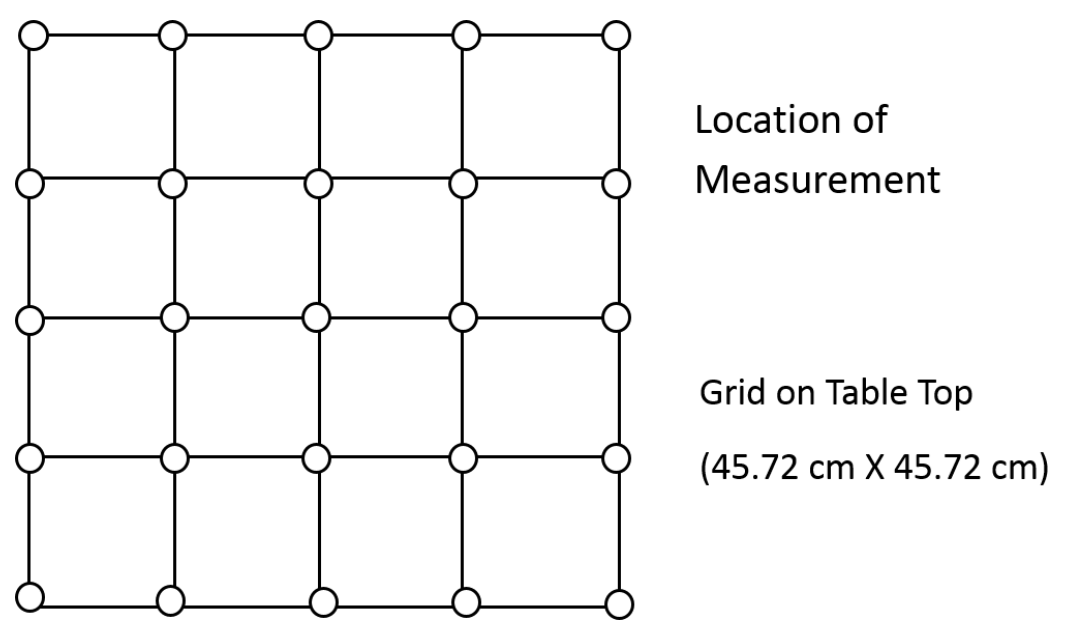

Figure 3. Table top placed within the anechoic chamber showing markings where a 3-axis field probe was placed. These 25 points were used for the $2.4 \mathrm{GHz}$ uniformity test.
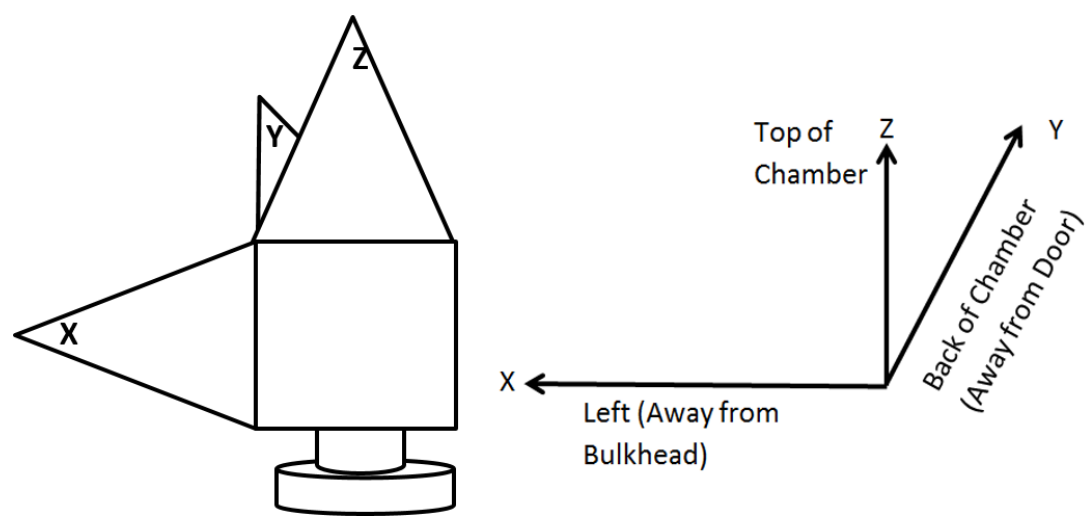

Figure 4. Field probe and orientation in chamber. 


\subsection{Effect of Antenna Type}

The type of antenna at the top of the anechoic chamber can affect the measured field uniformity. The measured field uniformity at the test platform for several antenna types is studied in this section. These antenna types are used throughout the document. The antenna types are listed in Table 2.

Table 2. Antennas and specifications.

\begin{tabular}{|c|c|c|c|c|}
\hline & Frequency Range of Operation & Gain & VSWR & Polarization \\
\hline Patch A & $2.4 \mathrm{GHz}-2.5 \mathrm{GHz}$ & $8 \mathrm{dBi}$ & $<1.5: 1 \mathrm{avg}$. & Left hand circular \\
\hline Patch B & $2.4 \mathrm{GHz}-2.5 \mathrm{GHz}$ & $8 \mathrm{dBi}$ & $<1.5: 1 \mathrm{avg}$. & Right hand circular \\
\hline Patch C & $2.4 \mathrm{GHz}-2.5 \mathrm{GHz}$ & $7 \mathrm{dBi}$ & $<1.5: 1$ & Right hand circular \\
\hline Horn & $1 \mathrm{GHz}-18 \mathrm{GHz}$ & $6 \mathrm{dBi}$ & $\begin{array}{c}3.5: 1 \mathrm{max} \\
\text { above } 1.5 \mathrm{GHz}\end{array}$ & Linear \\
\hline Helix & $2.4 \mathrm{GHz}-2.5 \mathrm{GHz}$ & $10 \mathrm{dBi}$ & $<1.3: 1$ & Right hand circular \\
\hline Discone & $25 \mathrm{MHz}-1300 \mathrm{MHz}$ & $2 \mathrm{dBi}$ & & Omnidirectional \\
\hline Patch D & $430 \mathrm{MHz}-450 \mathrm{MHz}$ & $7 \mathrm{dBi} 3 \mathrm{dBd}$ & $<1.5$ freq resonance & Right hand circular \\
\hline Patch E & $902 \mathrm{MHz}-928 \mathrm{MHz}$ & $9 \mathrm{dBi}$ & 1.3 & Left hand Circular \\
\hline
\end{tabular}

The VSWR was measured for each antenna to verify its performance with the specifications listed above. A vector network analyzer was connected through an anechoic chamber to each antenna and the $S_{11}$ parameter was measured. This was converted to VSWR and plotted for the frequency band of interest, as shown in Figure 5. 


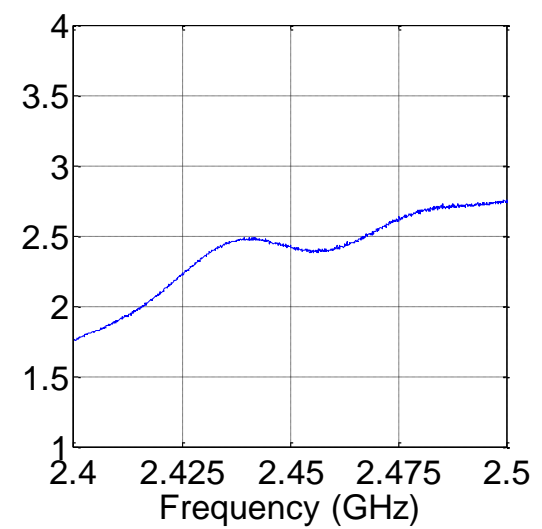

(a)

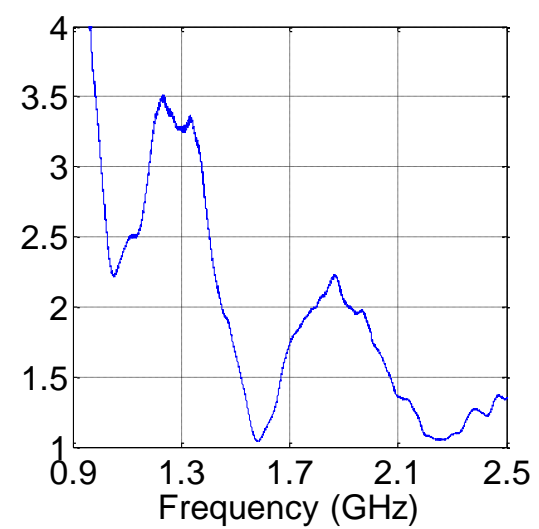

(c)

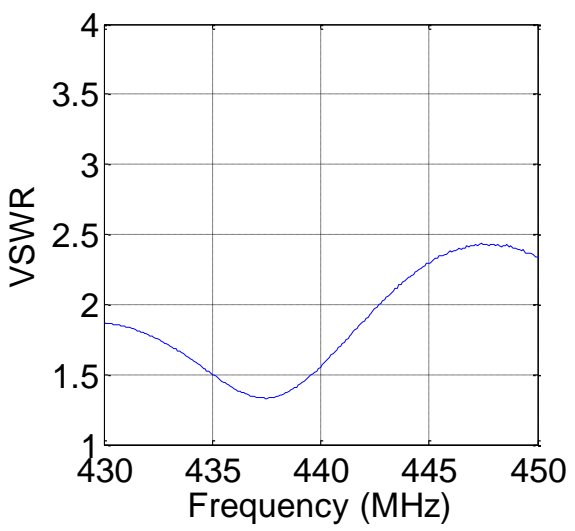

(e)

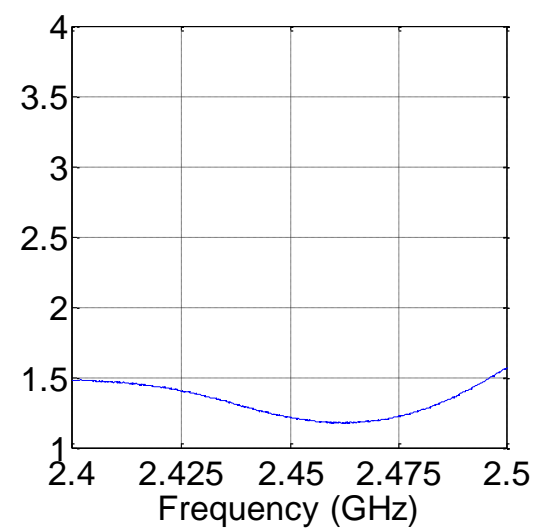

(b)

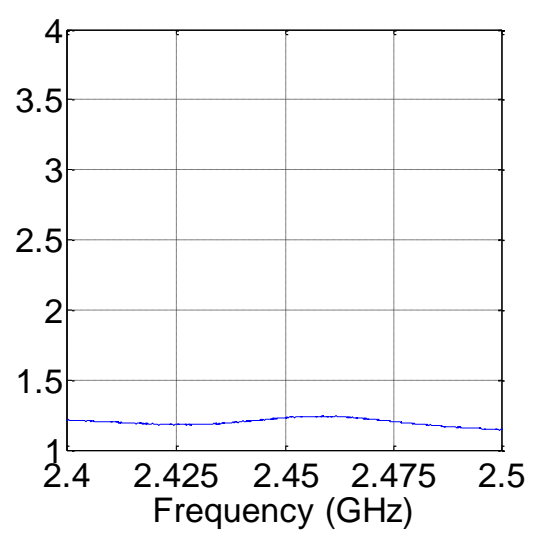

(d)

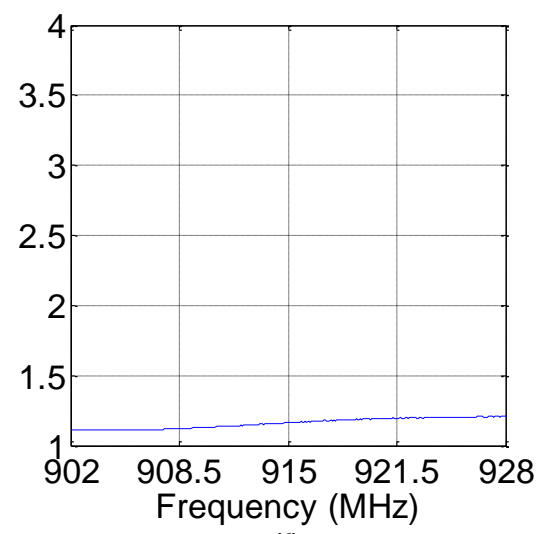

(f)

Figure 5. Measured VSWR of various antennas. (a) Patch B; (b) Patch C; (c) Horn; (d) Helix; (e) Patch D; and (f) Patch E. 


\subsection{NIST Field-Uniformity Measurement Results}

In the following sections we show contour plots using the data from the uniformity tests. The contour plots have an $\mathrm{x}$ and $\mathrm{y}$ axis labeled with measurements that replicate the dimensions of the platform within the chamber where the probe was placed. The contour plots take the coordinates of each point where the probe was placed and plots the respective 'heights' of the field strength. When only thirteen data points are used, a manual interpolation between points calculates the average value between points to help fill in the contour plot. With 25 points interpolation was not necessary. Different colors are filled in and the lines representing different strengths are labeled to help illustrate the differences in field strength.

\subsection{1 $462 \mathrm{MHz}$}

The lowest frequency tested in the anechoic chambers was $462 \mathrm{MHz}$. The setup as shown in Figure 1, with the 3 -axis field probe positioning shown in Figure 2 yielded the following successful results with less than $3 \mathrm{~dB}$ variation across the $30 \mathrm{~cm}$ by $30 \mathrm{~cm}$ square. Antenna D, as described in Table 3 was used for this test. Figure 6 below shows the contour plot of the uniformity results where the white circles mark the field probe measurement locations and linear interpolation was used between points. For this frequency, data for the measurement pattern in Figure 3 were not collected.

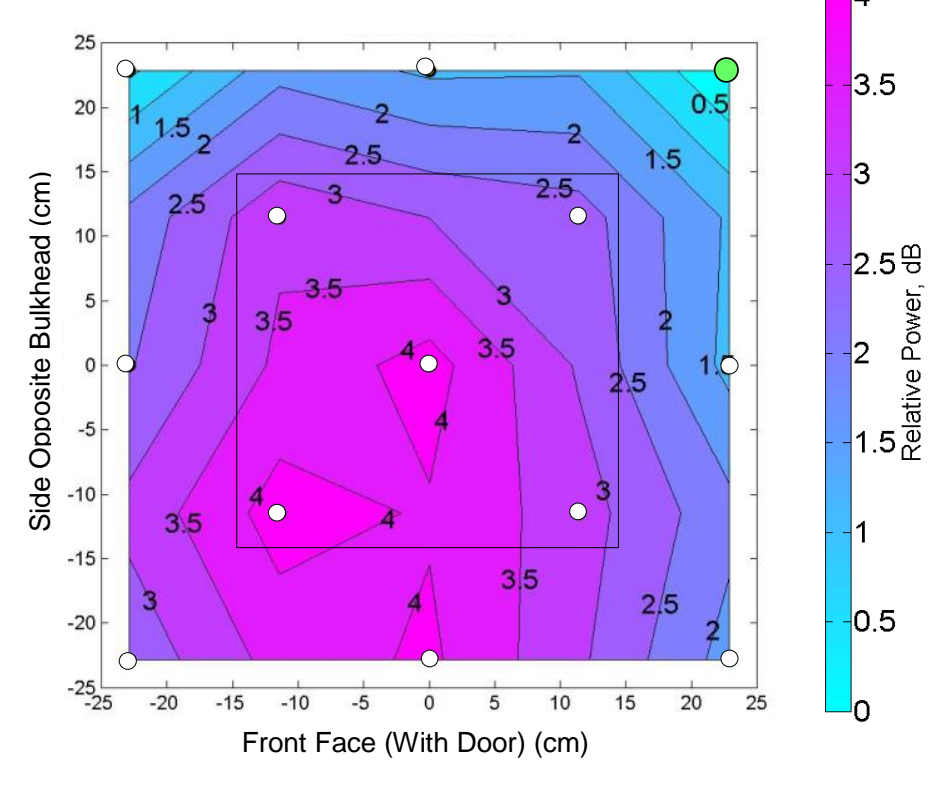

Selected data points

Minimum power point

Figure 6. Uniformity results for $462 \mathrm{MHz}$ antenna showing less than $3 \mathrm{~dB}$ variation across $30 \mathrm{~cm}$ by $30 \mathrm{~cm}$ square. 


\subsubsection{MHz}

The setup shown in Figure 1, with the 3-axis field probe positioning shown in Figure 2 produced the following successful results with less than $3 \mathrm{~dB}$ variation across the $30 \mathrm{~cm}$ by $30 \mathrm{~cm}$ square. Antenna E, as described in Table 3, was used in this test. Figure 7 shows the contour plot of the uniformity results. As in the $462 \mathrm{MHz}$ case, data for the measurement pattern in Figure 3 were not collected.

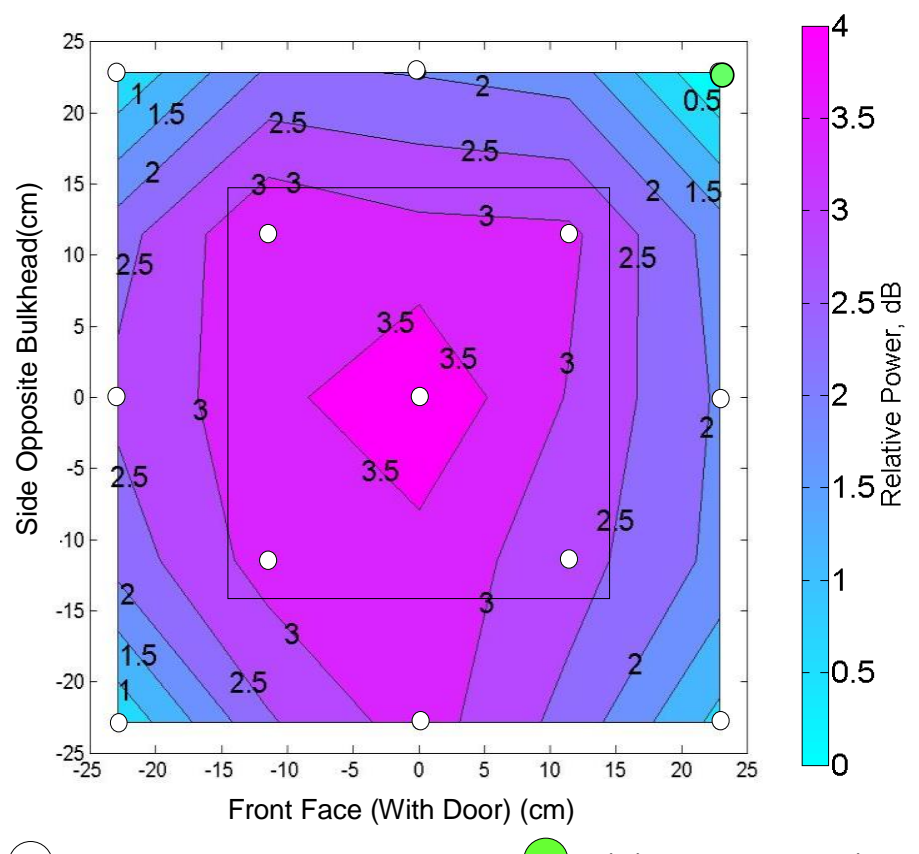

Selected data points

Minimum power point

Figure 7. Uniformity results for $915 \mathrm{MHz}$ antenna showing less than $3 \mathrm{~dB}$ variation across $30 \mathrm{~cm}$ by $30 \mathrm{~cm}$ square.

\subsection{GHz}

We determined the field uniformity at $2.4 \mathrm{GHz}$ using the approaches given in Figure 1 and Figure 3 with several different antennas shown in Table 2. This was necessary to determine the tradeoff between the maximum antenna gain and the field uniformity performance. In general, while high antenna gain is desirable, the uniformity suffers due to the narrowing of the main antenna beam. The first measurement case follows the data collection pattern and contour plot calculation, as in the previous sections.

In addition to the two data collection patterns, three different antennas were tested. These different antennas were mounted to the top of the chamber and the electric field was measured at the platform as shown in Figure 1. Patch A, Helix, and Patch C were tested. The antenna efficiency of the three antennas is not known.

Table 3 shows the various test configurations and conditions to verify field uniformity. Table 4 explains each test. Table 2 describes the parameters for each antenna used in Table 3 . Because the 
helix passed uniformity tests for the $2.4 \mathrm{GHz}$ band, it was used in the development of the test methods described in future sections.

Table 3. Various antennas and test configurations.

\begin{tabular}{|c|c|c|c|c|c|c|c|c|c|}
\hline $\begin{array}{c}\text { Antenna } \\
\text { Name }\end{array}$ & Antenna Type & $\begin{array}{c}\text { Test } \\
1\end{array}$ & $\begin{array}{c}\text { Test } \\
2\end{array}$ & $\begin{array}{c}\text { Test } \\
3\end{array}$ & $\begin{array}{c}\text { Test } \\
4\end{array}$ & $\begin{array}{c}\text { Test } \\
5\end{array}$ & $\begin{array}{c}\text { Test } \\
6\end{array}$ & $\begin{array}{c}\text { Test } \\
7\end{array}$ & $\begin{array}{c}\text { Test } \\
8\end{array}$ \\
\hline Patch A & Patch & $\ddot{n}$ & N/A & N/A & N/A & $\boldsymbol{n}$ & 2 & $\boldsymbol{N}$ & N/A \\
\hline Patch B & Patch & N/A & N/A & N/A & N/A & 2 & N/A & N/A & N/A \\
\hline Patch C & Patch & 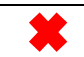 & N/A & N/A & N/A & $n$ & N/A & N/A & in \\
\hline Horn & $\begin{array}{c}\text { Dual Ridged } \\
\text { Horn } \\
\end{array}$ & N/A & N/A & N/A & N/A & 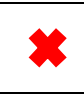 & N/A & N/A & 4 \\
\hline Helix & Spiral Helix & 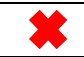 & 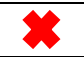 & $\bar{n}$ & $V$ & $\bar{n}$ & N/A & N/A & $v$ \\
\hline
\end{tabular}

Test was performed and the field uniformity exceeded $3 \mathrm{~dB}$ variation.

Test was performed and the field uniformity met the uniformity requirements.

Table 4. Uniformity test descriptions.

\begin{tabular}{|c|c|}
\hline $\begin{array}{c}\text { Test } \\
\text { Number }\end{array}$ & Description \\
\hline 1 & Uniformity field strength data with 13 collection points. \\
\hline 2 & Improvement upon bracket security with 13 collection points. \\
\hline 3 & 3 inch solid foam added to raise field probe with 13 collection points. \\
\hline 4 & Add RF absorber to top of chamber with 13 collection points. \\
\hline 5 & Uniformity field strength data with 25 collection points. \\
\hline 6 & Different physical antenna used (same model) with 25 collection \\
points.
\end{tabular}

\subsubsection{Uniformity Testing with 13 Collection Points}

The 3-axis field probe is placed on the platform according to Figure 2 and Figure 4 . The results are shown in Figure 8 through Figure 10 in the form of contour plots. In test 8 , and only for the $2.4 \mathrm{GHz}$ frequency, an aperture of dimensions $22.23 \mathrm{~cm}$ by $22.23 \mathrm{~cm}$ was made from a $47 \mathrm{~cm}$ by $47 \mathrm{~cm}$ piece of RF absorbing foam lens with depth $12.61 \mathrm{~cm}$ to reduce the interaction of the antenna's radiated signal and reflections from the walls of the chamber. 


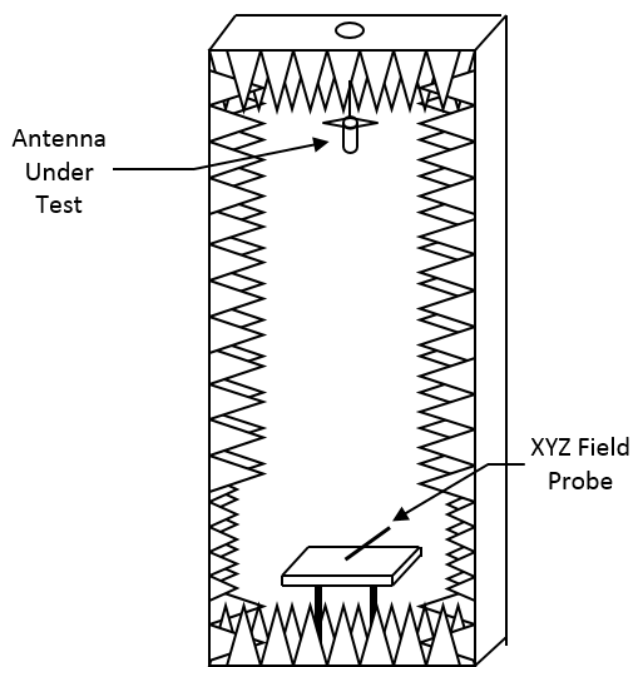

(a) Uniformity setup

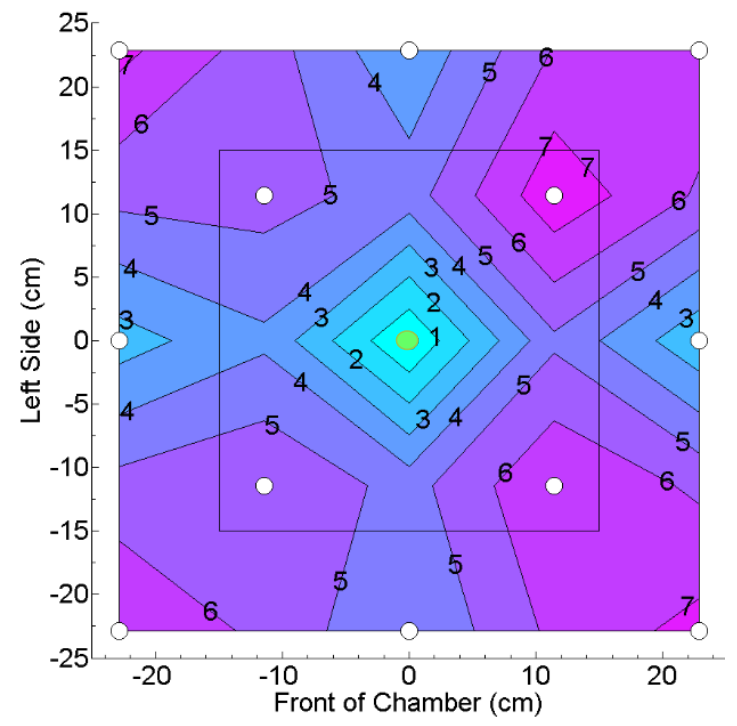

(c) Patch C

Selected data points

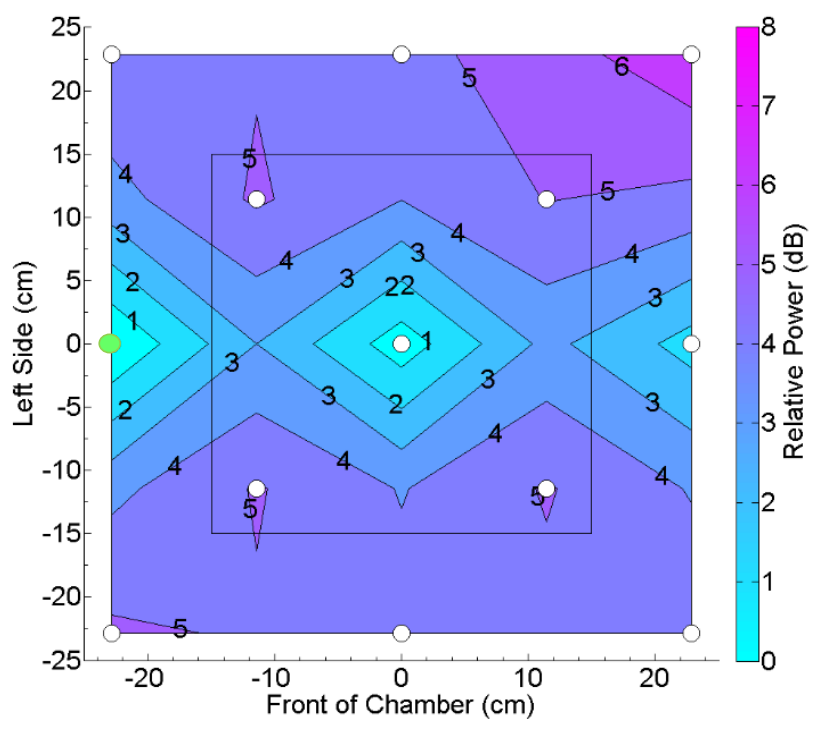

(b) Patch A

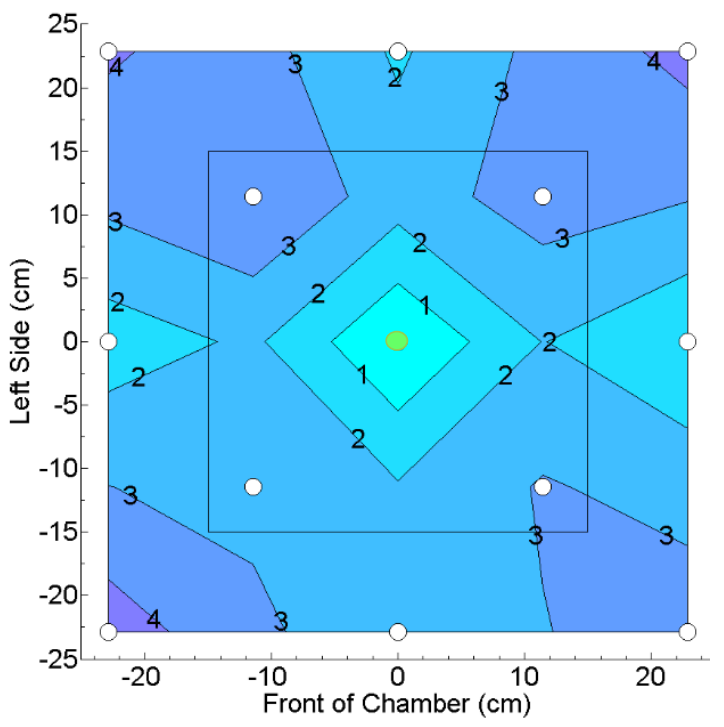

(d) Helix

Minimum power point

Figure 8. Uniformity results for Test 1 at $2.4 \mathrm{GHz}$.

\subsubsection{Uniformity Testing with 25 Collection Points}

The initial testing [2] was based on the collection of 13 data points and the computed contour plot. In order to simplify the verification of the field uniformity, we consider a different measurement process where we collect more data points, but only require a subset of those points to have less than $3 \mathrm{~dB}$ of variation. For the 25 point collection process, the 3 -axis field probe is placed on the platform according to Figure 3 and Figure 4. The goal here was to have $75 \%$ of the points on the platform within a $3 \mathrm{~dB}$ variation. This means up to six points can be removed, and if the remaining 
points are within $3 \mathrm{~dB}$ then the field uniformity can be considered successful. The following graphs show how many points would need to be removed in order for the remaining points to be within 3 $\mathrm{dB}$ variation. Many of the following figures show that more than six points would need to be removed to meet specifications. Only if six or less points are removed will the configuration pass the uniformity test.

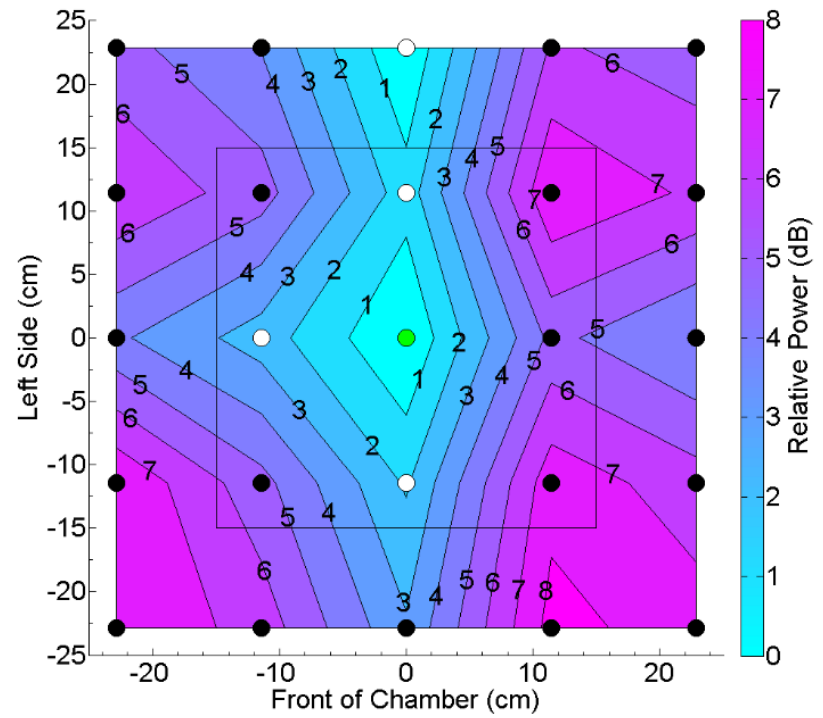

(a) Patch B

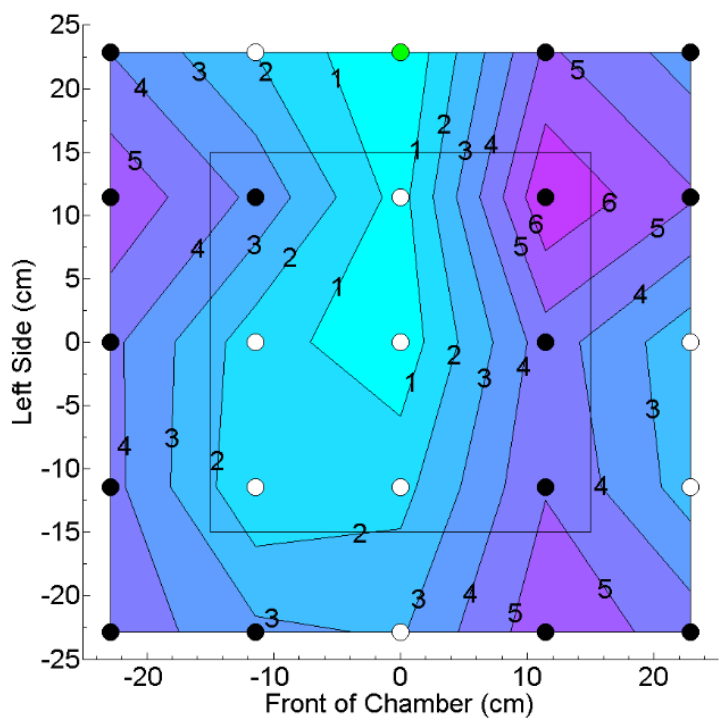

(c) Horn

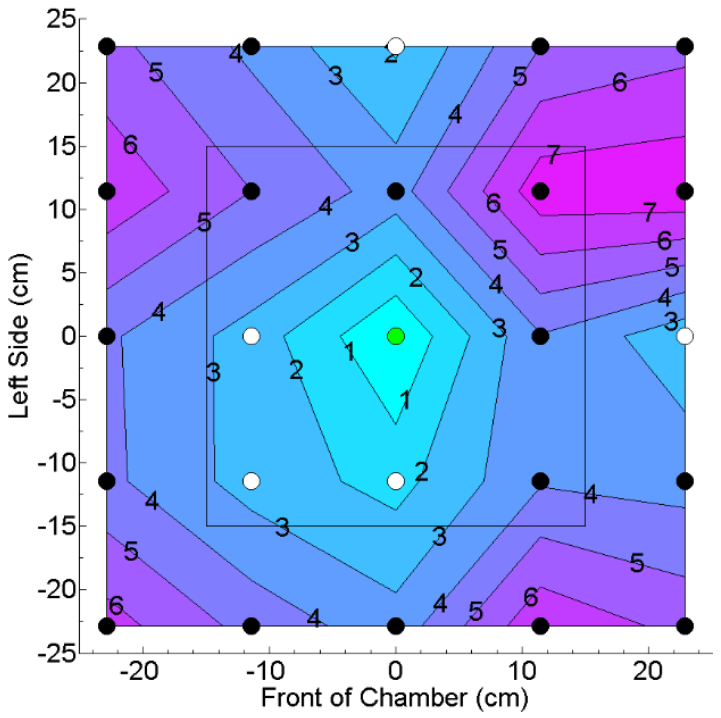

(b) Patch C

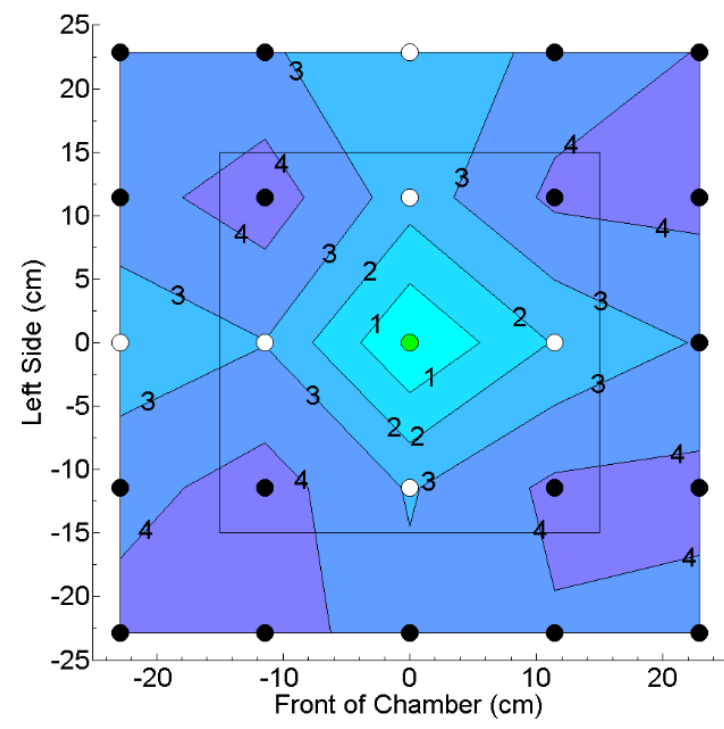

(d) Helix

Figure 9. Uniformity results for Test 5 at $2.4 \mathrm{GHz}$. Note that more than 6 points would have to be removed in all cases. These configurations will not pass the uniformity test. 


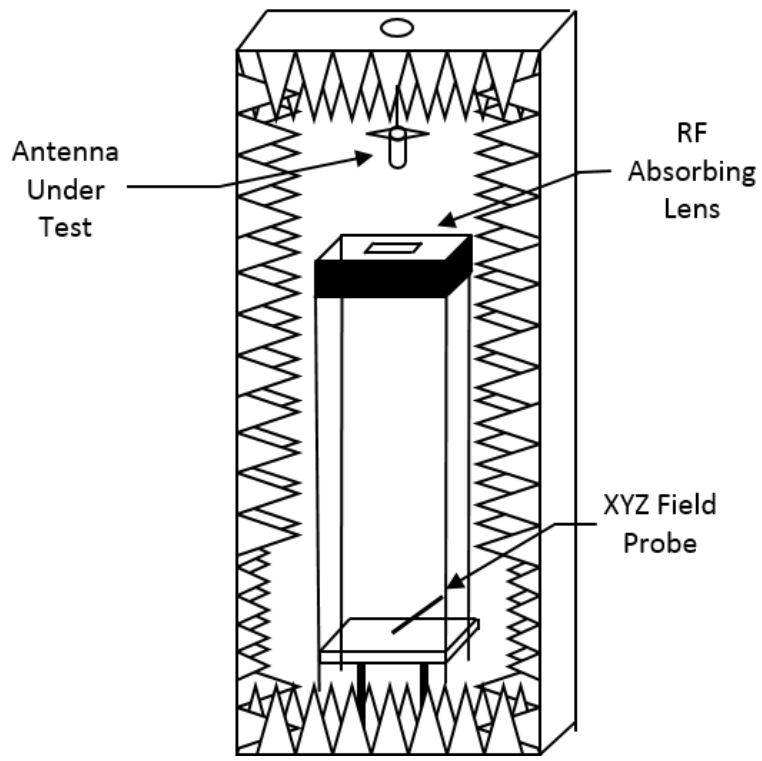

(a) Uniformity Setup

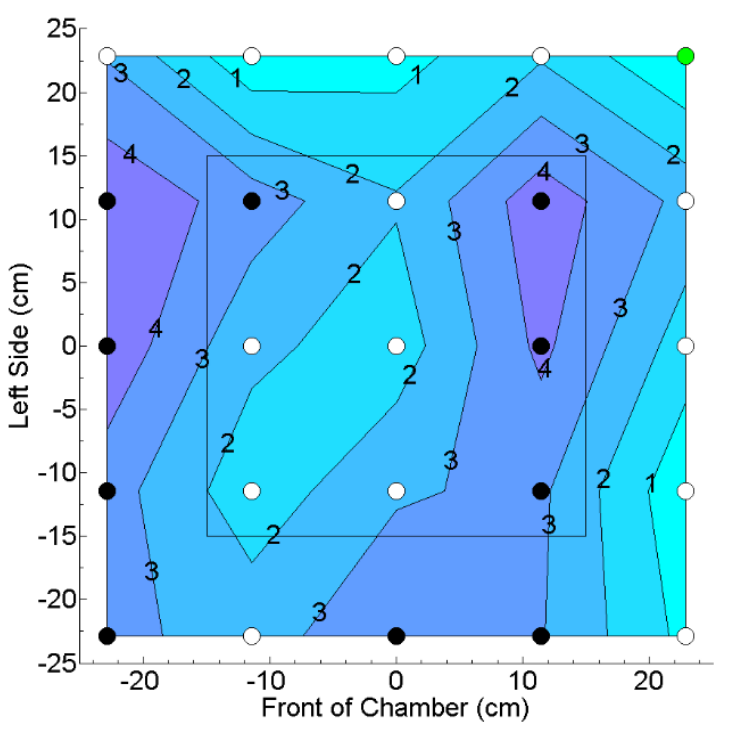

(c) Horn

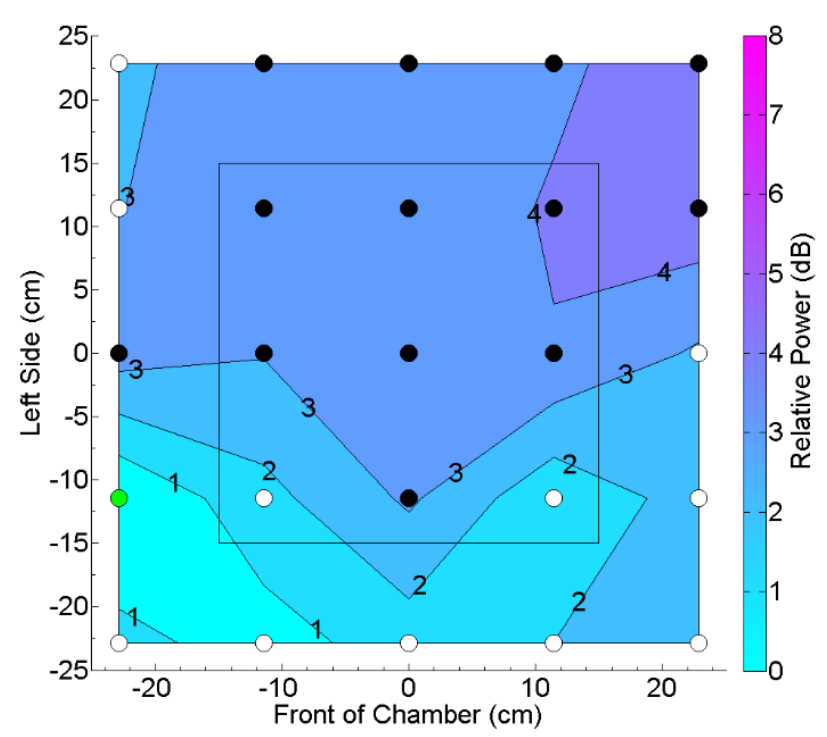

(b) Patch C

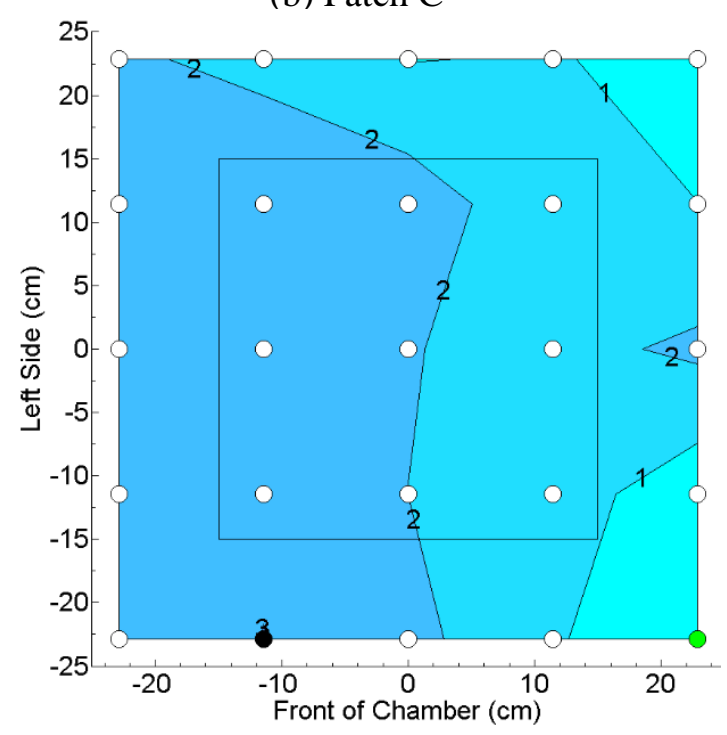

(d) Helix

Figure 10. Uniformity results for Test 8 at $2.4 \mathrm{GHz}$. Note that the Helix with the RF absorbing lens is the only configuration that requires less than 6 points to be removed and thus will pass the uniformity test.

\subsubsection{Conclusion}

Based on the series of measurements described above, we concluded that for the tests throughout this report, the Patch D would be used for the $450 \mathrm{MHz}$ frequency range, the Patch $\mathrm{E}$ would be used for the $900 \mathrm{MHz}$ frequency range, and for the $2.4 \mathrm{GHz}$ range the helix antenna with the RF 
absorbing foam lens would be used as the transmitting antenna at the top of the anechoic chambers. The latter is shown in Figure 10 (a). The following section provides an uncertainty analysis for the chosen (helix) antenna.

\subsection{Field Uniformity Measurement Uncertainty Analysis}

This section provides estimates of the uncertainties in the field uniformity measurements for a $2.4 \mathrm{GHz}$ helix antenna (one of the selected antennas for future tests). We performed this uncertainty analysis using a 3-axis field probe, helix antenna, signal generator, and amplifier. This analysis could be conducted by other labs to find the uncertainty in their field uniformity measurements.

Following the convention stated in [7], the uncertainties are broken into two categories, Type A (evaluated by statistical means), and Type B (evaluated by non-statistical means). Throughout this section are several graphs and tables showing components of the uncertainty analysis. Figure 11 shows repeat measurements of field uniformity test data in histogram form. Figure 11 (a) shows all data from Test 8 (described in Table 4). Figure 11 (b) shows all data from Test 4 (described in Table 4). Figure 11 (c) shows data within the region of interest for Test 8 . Figure 11 (d) shows data within the region of interest for Test 4. Figure 11 (e) shows combined data from Test 4 and Test 8. Figure 11 (f) shows combined data from the shared points (5 points in each test) in Test 4 and Test 8. Table 5 shows the mean, standard deviation, and relative uncertainty of the data plotted in Figure 11. Table 6 shows the different types of uncertainty contributions present in the field uniformity experiment. Figure 11 (f) was chosen as the most relevant experiment because it focuses on the region of interest for two separate experiments with common field measurement locations. The standard deviation and mean used in Section 2.4.3 are calculated from the data in Figure 11 (f).

\subsubsection{Type A Uncertainties}

Specifications on equipment (provided by the manufacturer) and measurement precision are considered Type A uncertainties and are found in Table 6. Two independent measurements (Tests 4 and 8 found in Table 4) were taken to find the field levels at various locations on the test platform within the anechoic chamber. This setup is shown in Figure 1.

\subsubsection{Type B Uncertainties}

Specifications on equipment utilized in the setup (not provided by the manufacturer) are considered Type B uncertainties, and are found in Table 6. For the configuration tested here, the uncertainty contributions due to the repeatability of the signal generator, amplifier, and cable are considered Type B uncertainties because the uncertainty was approximated from the difference in the average of two data sets.

\subsubsection{Combined Uncertainty}

Both types of uncertainties are combined to determine the total uncertainty in the field uniformity measurements. The calculation of relative uncertainty in Table 5 uses the following equation:

$$
u_{\text {relative }}(\mathrm{dB})=20 \times \log _{10}\left(1 \pm \frac{\sigma}{\mu}\right),
$$


where $\sigma$ is the standard deviation and $\mu$ is the mean.
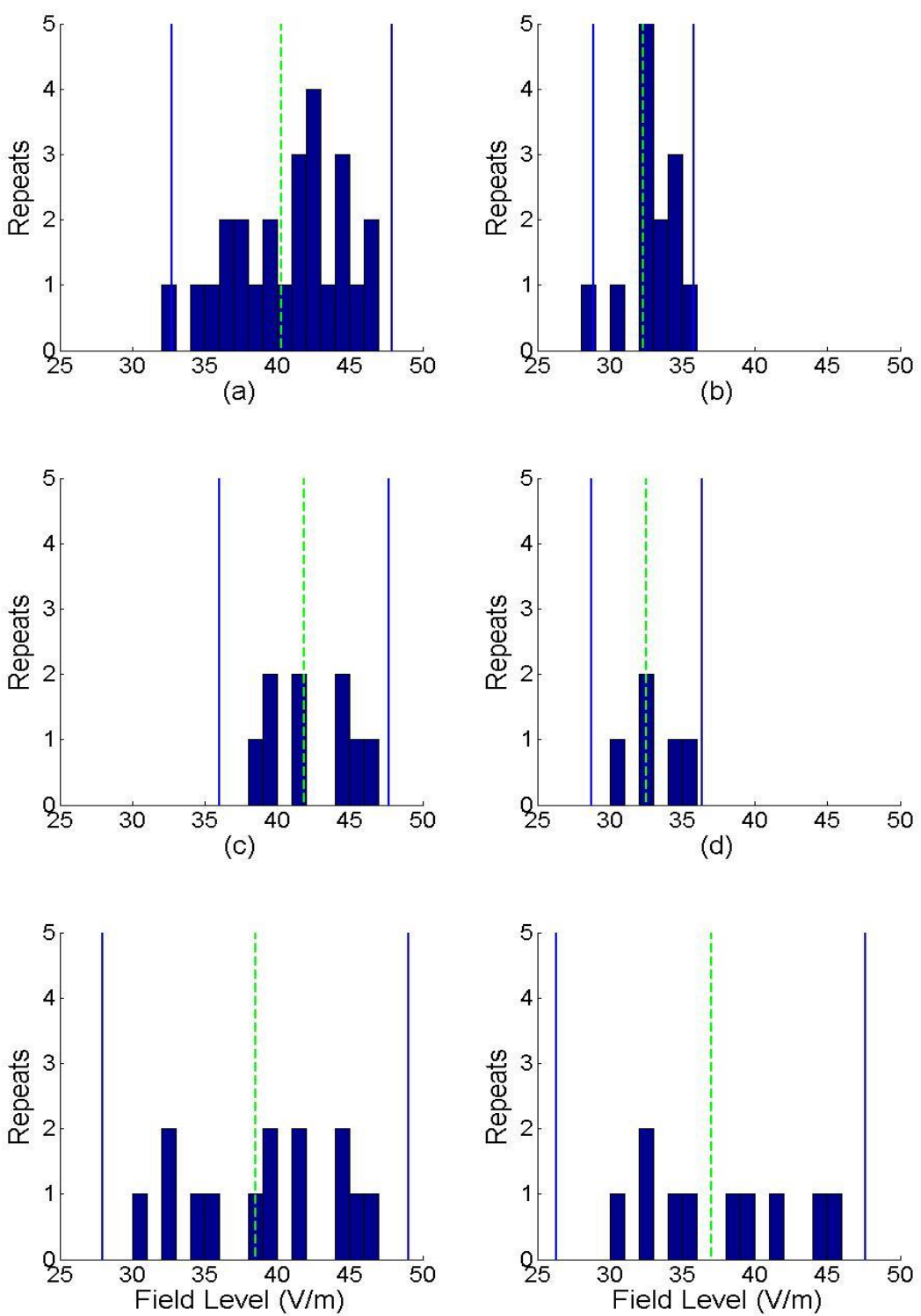

(e)

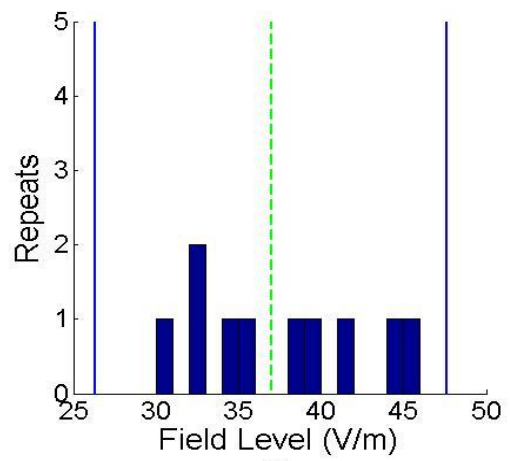

(f)

Data ---Mean - +/- 2 Standard Deviations

Figure 11. (a) Test 8 (b) Test 4 (c) Test 8 Region of Interest (d) Test 4 Region of Interest (e) Test 4 and Test 8 Region of Interest (f) Shared Points of Test 4 and Test 8

Calculations in Table 5, excluding $u_{\text {relative }}$, use (3):

$$
u(\mathrm{~dB})=20 \times \log _{10}\left(\frac{\text { linear value }}{\frac{1 \mathrm{v}}{\mathrm{m}}}\right)
$$


Table 5. Mean $(\mu)$, standard deviation $(\sigma)$, and difference of mean, for subplots shown in Figure 11. Note that relative uncertainty $(u)$, is not symmetric about the mean because of the log conversion process.

\begin{tabular}{|c|c|c|c|c|c|c|}
\hline Subfigure & $(\mathbf{a})$ & $(\mathbf{b})$ & $(\mathbf{c})$ & $(\mathbf{d})$ & $(\mathbf{e})$ & $(\mathbf{f})$ \\
\hline$\mu(\mathrm{V} / \mathrm{m})$ & 40.3 & 32.3 & 41.8 & 32.5 & 38.5 & 37.0 \\
\hline$\mu(\mathrm{dBV} / \mathrm{m})$ & 32.1 & 30.2 & 32.4 & 30.2 & 31.7 & 31.4 \\
\hline$\sigma(\mathrm{V} / \mathrm{m})$ & 3.8 & 1.7 & 2.9 & 1.9 & 5.3 & 5.3 \\
\hline$u_{\text {relative }}(\mathrm{dB})$ & +0.78 & +0.45 & +0.58 & +0.49 & +1.12 & +1.16 \\
& -0.86 & -0.47 & -0.62 & -0.52 & -1.29 & -1.34 \\
\hline
\end{tabular}

Calculations in Table 6 use (4):

$$
\mu, \sigma(\mathrm{dB})=20 \times \log _{10}(\text { linear value }+1)
$$

Table 6. Description of measurement uncertainties with associated values.

\begin{tabular}{|c|c|c|c|c|c|}
\hline Type & Variable Name & $\begin{array}{l}\text { Uncertainty } \\
\text { Description }\end{array}$ & Method of Estimate & $\begin{array}{c}\text { Values } \\
\text { (Linear) }\end{array}$ & $\begin{array}{c}\text { Values } \\
\text { (dB) }\end{array}$ \\
\hline A & $u_{\text {precision }}$ & $\begin{array}{c}\text { Precision in } \\
\text { Measurements }\end{array}$ & $\begin{array}{l}\text { Relative uncertainty from the } \\
\text { standard deviation of } \\
\text { measurements from Test } 4 \text { and } \\
\text { Test } 8 \text { (see Table } 4) \text {. }\end{array}$ & 0.17 & $\begin{array}{l}+1.16 \\
-1.34\end{array}$ \\
\hline A & $u_{\text {probe }}$ & $\begin{array}{l}\text { EMC Field } \\
\text { Probe }\end{array}$ & Specified by manufacturer. & 0.1 & $\begin{array}{l}+0.9 \\
-0.9 \\
\end{array}$ \\
\hline B & $u_{\text {signal generator }}$ & $\begin{array}{l}\text { Signal } \\
\text { Generator, } \\
\text { Amplifier, } \\
\text { Cable }\end{array}$ & $\begin{array}{c}\frac{\left(\mu_{5 a}-\mu_{5 b}\right)+\left(\mu_{5 c}-\mu_{5 d}\right)}{2} \\
\text { Where } \mu_{5 a} \text { is the mean } \mathrm{dB} \text { value } \\
\text { listed in Table } 5 \text { referring to } \\
\text { Figure } 11 \text { (a) etc. }\end{array}$ & 0.26 & 2.0 \\
\hline
\end{tabular}

The linear values in Table 6 are used in the following equation to determine the combined uncertainty.

$$
u_{\text {combined }}=\sqrt{u_{\text {precision }}^{2}+u_{\text {probe }}^{2}+u_{\text {sig gen unit }}^{2}}
$$

We combine these uncertainties using root-sum-of-squares addition on the linear values and then converted back to decibels. The combined uncertainty converted to decibels is $2.48 \mathrm{~dB}$, which is within $3 \mathrm{~dB}$, where $3 \mathrm{~dB}$ is the threshold for field uniformity specified in NFPA 1982.

\section{Multipath Test and Reverberation Chambers}

\subsection{Introduction}

This section covers the basics of calibration, setup, and measurements for electromagnetic reverberation chambers to simulate a fireground response scenario in which the incident command station is outside a structure and a portable unit is inside a highly-reflective structure such as an oil refinery or factory. As in [1], both the base station and the portable device are tested under freefield conditions and, to simulate building penetration, $\sim 100 \mathrm{~dB}$ of average path loss is included 
between the two units. The intent is to provide the reader with a basic methodology on how to carry out measurements of wireless systems wherein one of the communication nodes is a wireless device located in an electromagnetic reverberation chamber (RC) and the other is located in an anechoic chamber (AC). The reverberation chamber in this test and others throughout this technical note is 147.32 meters (58 inches) high, $118.11 \mathrm{~cm}$ (46.5 inches) long, and $149.86 \mathrm{~cm}$ (59 inches) wide. Chambers with other dimensions can be used so long as they are rated for the frequency of the device under test. Any high efficiency antenna, such as a Helix, can be used in this multipath test because the reverberation chamber averages out the antenna pattern. This means the antenna pattern will not affect the result. Figure 12 shows the basic test setup. An implicit assumption in this discussion is that the wireless system utilizes integrated or non-removable antennas on the RF PASS. The wireless system and the RF PASS may also have limited, if any, self-diagnostic capabilities. Further, in-depth discussion on key RC parameters can be found in the supplied references.

Throughout this section there are many equations used with variable descriptions that can be found in Table 7.

Table 7. Variable descriptions.

\begin{tabular}{|c|c|}
\hline Variable & Description \\
\hline$L_{\mathrm{AC}}^{\mathrm{SG}}$ & $\begin{array}{l}\text { Loss of Cable Connected between the Signal Generator and Anechoic } \\
\text { Chamber }\end{array}$ \\
\hline$L_{\mathrm{AC}}^{\mathrm{Tx}}$ & $\begin{array}{l}\text { Loss of Cable Connected between the Transmitting Antenna and the } \\
\text { Anechoic Chamber }\end{array}$ \\
\hline$L_{\mathrm{RC}}^{\mathrm{Rx}}$ & $\begin{array}{l}\text { Loss of Cable Connected between the Receiving Antenna and the } \\
\text { Reverberation Chamber }\end{array}$ \\
\hline$L_{\mathrm{RC}}^{\mathrm{SA}}$ & $\begin{array}{l}\text { Loss of Cable Connected between the Spectrum Analyzer and the } \\
\text { Reverberation Chamber }\end{array}$ \\
\hline$L_{t o t}$ & Total cable Loss \\
\hline$e_{\mathrm{Tx}, \mathrm{Rx}}$ & Antenna Efficiency between Transmitting and Receiving Antenna \\
\hline$m_{\mathrm{Tx}, \mathrm{Rx}}$ & Mismatch Loss between Transmitting and Receiving Antenna \\
\hline$P_{\mathrm{SA}}^{\mathrm{ref}}(i)$ & Peak Spectrum Analyzer Measurement for each paddle position (i) \\
\hline$G_{C C}$ & Chamber-to-Chamber Gain \\
\hline$P_{\mathrm{SG}}^{\mathrm{ref}}$ & Power Setting of the Signal Generator \\
\hline$P_{\mathrm{SA}}^{\mathrm{ref}}$ & Power Measurement from the SA \\
\hline$G_{\mathrm{AMP}}$ & Gain of the Optional Amplifier \\
\hline$\langle\cdot\rangle$ & Average of Measurements across all Paddle Positions \\
\hline$P L_{\text {Target }}$ & Target Average-Total-Attenuation \\
\hline$L_{V A R}$ & Variable Attenuation loss \\
\hline$G_{T x}$ & Gain of Transmitting Antenna \\
\hline
\end{tabular}




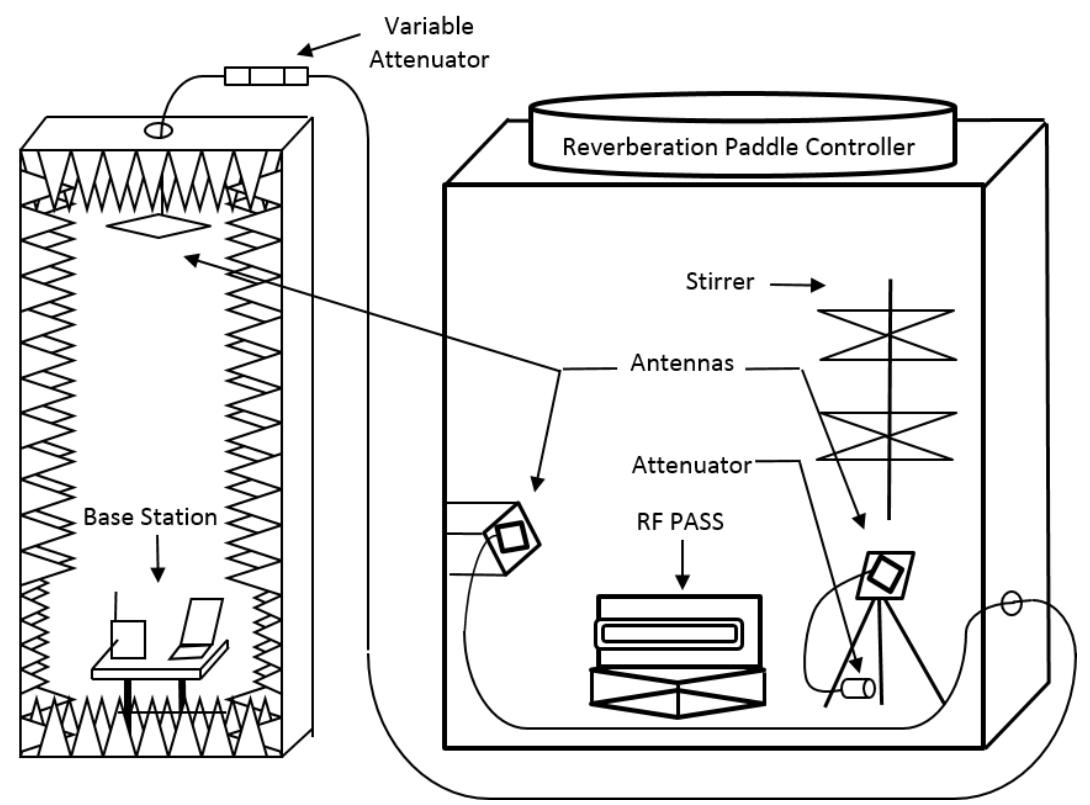

Figure 12. General multipath test setup showing linked anechoic and reverberation chambers. The RF PASS unit is located on a solid foam platform to ensure a minimum of a $1 / 2 \lambda$ separation from any of the chamber walls or floor. Note the Rx antenna in the RC is only used during the calibration setup discussed below; the Rx antenna and cable are left in the RC, but disconnected from the bulkhead during testing of the RF PASS.

An important focus of the discussion below is on the use of a signal generator and spectrum analyzer in the calibration process. Vector network analyzers are often used to calibrate RCs as well. The calibration here offers another option when use of a vector network analyzer is not feasible. This calibration process is intended for use in setting up the multipath test environment inside a RC.

\subsection{Background}

The basic theory behind RCs is well established [8, 9], and RCs are used in several wireless technology measurements such as electromagnetic compatibility (EMC) testing [6] and total radiated power (TRP) of single-frequency [10][11], and modulated-signal [12][13] systems. The application discussed here focuses on use of the $\mathrm{RC}$ to create a rich multipath environment to emulate expected conditions when deployed in settings that provide a significant amount of electromagnetic scattering and reflections (e.g., factory floors, oil refineries, etc [14][15]). A key consideration relevant to wireless system testing is that the $\mathrm{RC}$ is considered a statistical RF environment. This means that statistical analysis techniques are required to interpret measurement results from a measurement process that makes use of a RC. The calibration process is discussed below and will demonstrate how statistical analysis techniques apply to RCs.

Reverberation chambers operate under a proposition directly counter to that of an electromagnetic anechoic chamber, namely, that nearly all the energy is reflected back from the chamber walls and into the testing area or volume. In an anechoic chamber, the absorbing material on the walls prevents (or at least significantly attenuates) any electromagnetic reflections scattered from the 
wall surfaces. Additionally, in a RC, the mixing of the electromagnetic fields by "stirring" via rotating paddles or position changes creates a rich multipath environment.

\subsection{Quality of the Reverberation Chamber}

The RC test setup requires a calibration process to ensure accurate results are achieved during testing. This calibration process assumes the RC is "well-stirred". A well-stirred chamber implies that the chamber supports statistically uniform and isotropic electric and magnetic fields, (in all orthogonal field components), and that these field components vary sufficiently in amplitude (e.g., a coefficient of variation of one [16]) and over a sufficient dynamic range in level (e.g., greater than $20 \mathrm{~dB}$ ) during the mixing process. (Note that henceforth we will discuss RC behavior with respect to the electric field or power since those are two quantities that are often measured in a RC.)

In [16], the concept of a good or well-stirred reverberation chamber is discussed along with three measurements or checks that can be used to evaluate the quality of the RC setup. The three measurements are all statistical in nature, namely the maximum-to-average ratio, the maximumto-minimum ratio and the coefficient of variation. Achieving "good" values for these three measures does not guarantee a well-stirred chamber setup. If the results from these tests are not acceptable, the RC setup should be reworked and re-evaluated as needed. Manufacturers of RCs typically provide a range of frequencies over which the $\mathrm{RC}$ is considered well-stirred. For the purposes of the multipath test described below, $\pm 3 \mathrm{~dB}$ variation in electric field is considered wellstirred. However, we need to recognize that, while a nearly empty RC may perform quite well, when objects such as test equipment, antennas, and the RF PASS are placed in the RC, the stirring quality can degrade quite significantly. A detailed process for determining the quality of the RC setup is described in [6].

\subsection{Calibration Process}

This test procedure begins with a determination of the $L_{t o t}$ in which a known source such as a signal generator $(\mathrm{SG})$ and a receiver such as a spectrum analyzer (SA) are used to find the gain between a transmitting source and the receiver. This is the gain of the chamber-to-chamber environment, and in this context, a negative gain value (in $\mathrm{dB}$ ) represents a loss. Since a RC is one chamber of the system, a stirring paddle is turned between measurement samples to produce statistically uncorrelated samples, which are then averaged. (Note that uncorrelated samples imply independence between those samples in a well-stirred RC.) The procedure follows below. All equipment that will be located in the $\mathrm{RC}$ as part of the test should be present in the RC during the calibration process. This includes the inactive (powered down) RF PASS.

\section{Additional Calibration Considerations}

We need to first observe the RF PASS waveform as well as the base station waveform to find the appropriate hold time and measurement bandwidth for the spectrum analyzer. If the signal utilizes a narrow frequency band, for example less than $25 \mathrm{kHz}$, calibrating the chamber based on the center frequency of the band is sufficient. In general, the spectrum analyzer should be set at a 
frequency step and resolution bandwidth between $10 \mathrm{kHz}$ and $250 \mathrm{kHz}$ for communication systems operating below $6 \mathrm{GHz}$. For larger bandwidths, a larger frequency step can be used. The minimum frequency step should be smaller than the carrier (or subcarrier) frequency spacing.

This calibration process does not determine the coherence bandwidth of the RC. In an AC, the coherence bandwidth is essentially the usable frequency range of the AC. By contrast, the coherence bandwidth in an unloaded RC, such as that used here, is generally quite narrow, e.g., often less than $1 \mathrm{MHz}$. For this particular multipath test, the environment essentially represents the worst-case scenario in which many reflections are present. A process for determining the RC coherence bandwidth based on vector network analyzer measurements is discussed in [12] and [17].

\subsection{Multipath Testing of an RF PASS System}

For RF PASS testing, the configuration for the multipath test consists of an AC connected to a RC. In this case, the calibration process should include all elements used in the actual testing. In addition, the RF PASS is placed in the RC so that the loading effects on the chamber-to-chamber gain are included. The variable attenuation is set to $0 \mathrm{~dB}$, in other words, no additional attenuation is added during the calibration process. Throughout this section various components are referred to as having gain, or loss. A component has loss if the signal strength decreases after traveling through the component (e.g. cables). A component has gain if the signal strength increases after traveling through the component (e.g. amplifier).

\section{Step 1. Setting the Calibration Source}

The first step is to set up the overall system so that average total attenuation between the testing plane of the anechoic chamber and the RF PASS in the reverberation chamber is set to the desired target level, which in this NFPA test is $100 \mathrm{~dB}$. The first step in the process is to directly connect the signal generator ( $\mathrm{SG}$ ) to the spectrum analyzer (SA) through the cables that will be used to connect to the antennas used for calibration (see Figure 13). Note that there are two sets of antennas; those used to link the two chambers and those used for the calibration process.

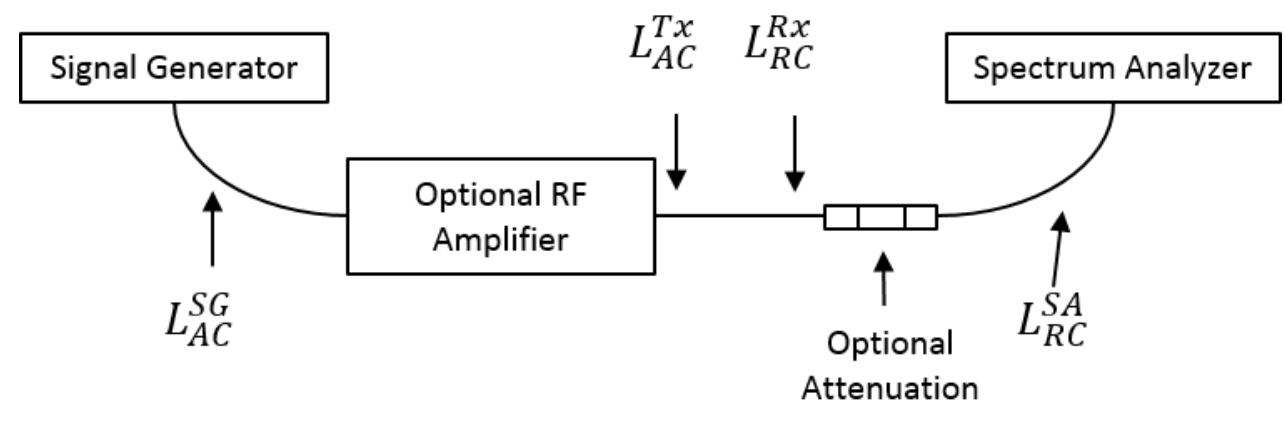

Figure 13. Initial step in establishing multipath test setup. The signal generator (SG) is directly connected to the spectrum analyzer (SA) through the cables used in the next step of the calibration process.

An important consideration is that the dynamic range between the SG and the SA must be at least $120 \mathrm{~dB}$. The dynamic range is the difference (in power) between the output power of the SG and the noise floor of the SA. Thus, we may need to include an amplifier directly after the SG. For 
example, if the $\mathrm{SG}$ output is $+10 \mathrm{dBm}$ and the SA noise floor is $-100 \mathrm{dBm}$, the dynamic range is $110 \mathrm{~dB}$. In order to achieve $120 \mathrm{~dB}$ of dynamic range, $10 \mathrm{~dB}$ of gain is added from the optional RF amplifier. The optional attenuation may be necessary to lower the resulting output power from the amplifier in order to protect the SA input during the initial setup in steps 1.1 and 1.2 below; this optional attenuation is not used when connected via the two chambers discussed below. For variable definitions see Table 7 .

1.1 Connect all the cables in the path between the signal generator and the spectrum analyzer (i.e., connect the cable connecting the transmitter and anechoic chamber directly to the cable connecting the transmitter and reverberation chamber), omitting the Tx and Rx antennas.

1.2 Measure the total cable loss $L_{\text {tot }}$ between the SG and the SA. The loss value is negative (in $\mathrm{dB}$ ) quantities, or between 0 and 1 (linear). $L_{\mathrm{tot}}[\mathrm{dB}]=L_{\mathrm{AC}}^{\mathrm{SG}}[\mathrm{dB}]+L_{\mathrm{RC}}^{\mathrm{SA}}[\mathrm{dB}]+L_{\mathrm{AC}}^{\mathrm{Tx}}[\mathrm{dB}]+L_{\mathrm{RC}}^{\mathrm{Rx}}[\mathrm{dB}]=$ total cable losses in $\mathrm{dB}$ or in the linear scale, $L_{\text {tot }}=L_{\mathrm{AC}}^{\mathrm{SG}} L_{\mathrm{RC}}^{\mathrm{SA}} L_{\mathrm{AC}}^{\mathrm{Tx}} L_{\mathrm{RC}}^{\mathrm{Rx}}$.

\section{Step 2. Chamber-to-Chamber Gain Measurement for a Signal Generator and a Spectrum Analyzer}

The second step requires connection between the two chambers. Figure 14 shows the calibration setup with the two chambers. Initially, the variable attenuation is set to $0 \mathrm{~dB}$.

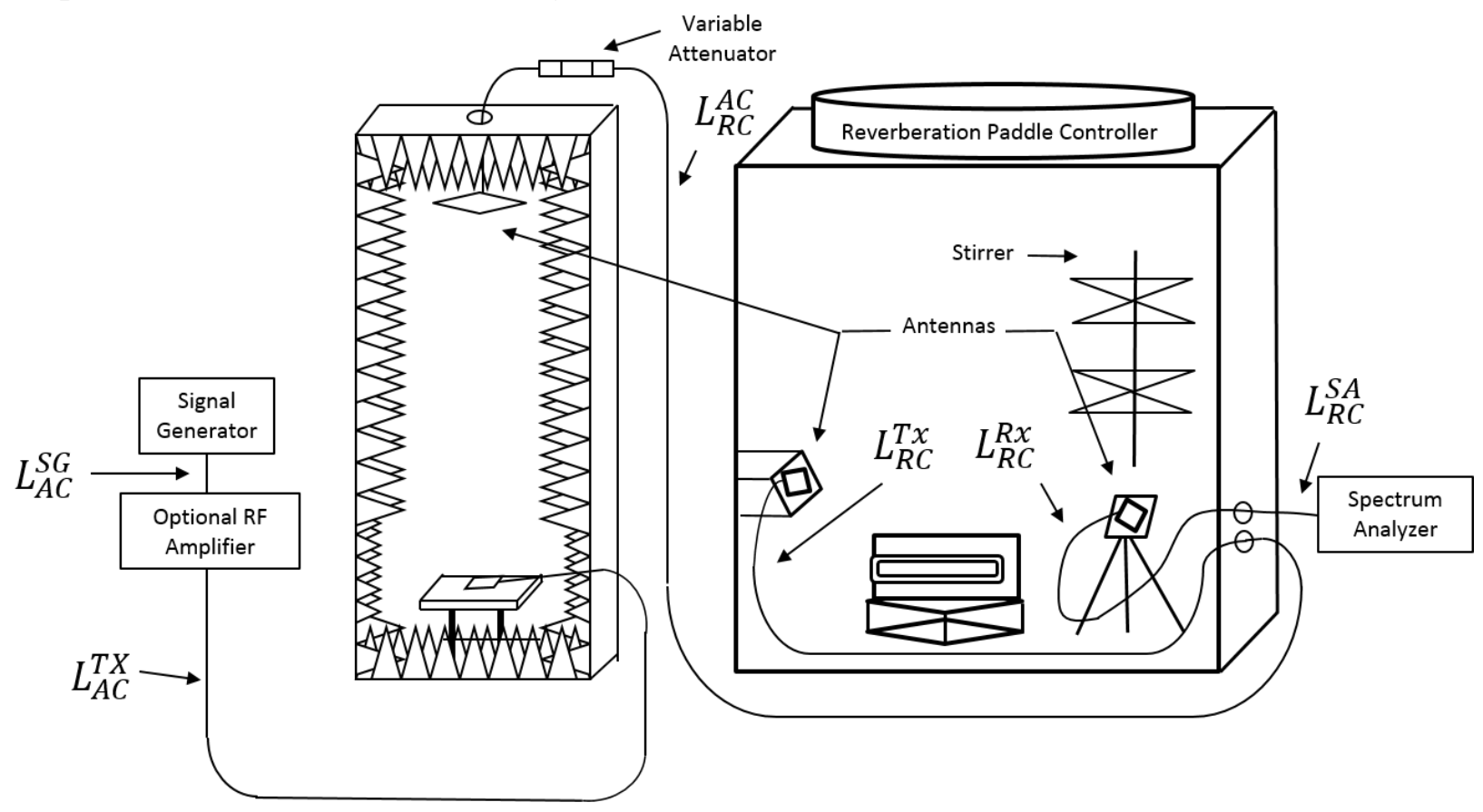

Figure 14. The calibration setup with the SG connected to the Tx antenna in the anechoic chamber and the SA connected to the Rx antenna in the reverberation chamber. The optional amplifier is necessary if the SG cannot supply adequate signal power. The cables in this figure are the same as used in Figure 13.

Within the RC, connect two antennas, one to the cable connecting the $\mathrm{AC}$ and the $\mathrm{RC}$, and one to the SA. The main beam of the antenna patterns should point away from each other, and the main beam of the RC to AC antenna (in Figure 14) should point towards a stirrer. The goal in setting up the orientation is to minimize coupling (i.e., direct electromagnetic interactions) between the antennas. Typically, the antennas are placed at least $2 \lambda$ apart. 
Place the RF PASS (powered down) in the RC chamber to determine the effects that its presence has on the RC gain.

Determine the antenna efficiencies $\left(e_{\mathrm{Tx}, \mathrm{Rx}}\right)$ and mismatch loss $\left(m_{\mathrm{Tx}, \mathrm{Rx}}\right)$. These parameters may be provided by the manufacturer or measured with industry accepted measurement techniques. If the antenna efficiencies are not known, we can preferably use an antenna, well-accepted by the wireless testing community such as a dual ridged or standard gain horn, which will typically have high-efficiency values.

Collect the peak SA measurement $P_{\mathrm{SA}}^{\mathrm{ref}}(i)$ for each paddle position, $i$, across the frequency band of interest. As discussed in [14], [11], the number of paddle positions impacts the uncertainty analysis. For this particular test, between 70 to 100 independent paddle positions provide a reasonable level of average field uncertainty.

Calculate the chamber-to-chamber gain $G_{\mathrm{CC}}$ for the desired configuration, as discussed below:

The equation used to calculate $G_{\mathrm{CC}}$ as a function of frequency is

$G_{\mathrm{CC}}(f)[\mathrm{dB}]=-\mathrm{L}_{\mathrm{tot}}(f)[\mathrm{dB}]+\left\langle P_{\mathrm{SA}}^{\mathrm{ref}}(f)[\mathrm{dB}]\right\rangle-P_{\mathrm{SG}}^{\mathrm{ref}}(f)[\mathrm{dB}]-G(f)_{\mathrm{AMP}}[\mathrm{dB}]$

where

$P_{\mathrm{SG}}^{\mathrm{ref}}=$ power setting of the $\mathrm{SG}$;

$P_{\mathrm{SA}}^{\mathrm{ref}}=$ power measurement from the SA;

$G_{\mathrm{AMP}}[\mathrm{dB}]=$ gain of the optional amplifier;

$\langle>$ denotes an ensemble average

The mean chamber-to-chamber gain over the band of interest is found by averaging $G_{C C}(f)$ over frequency. If the cable losses are considered constant over the frequency band of interest, and the power supplied by the signal generator is constant over that frequency band, then only the received power $P_{\mathrm{SA}}^{\mathrm{ref}}(f)$ is a function of frequency.

An alternative way to express (6) is

$G_{\mathrm{CC}}[\mathrm{dB}]=10 \times \log _{10}\left[\frac{\left\langle P_{\mathrm{SA}}^{\mathrm{ref}}\right\rangle}{P_{\mathrm{SG}}^{\mathrm{ref}}} \times \frac{1}{L_{\text {tot }}}\right]$,

where the cable losses and power levels are power losses expressed on a linear (rather than log) scale. The frequency dependence is not explicitly indicated in (7).

\section{Refined Chamber-to-Chamber Gain Measurement}

In order to improve the measurement accuracy, the antenna efficiencies and mismatch characteristics can be included, if known. This may be particularly beneficial if the mismatches are high or the efficiencies are low (or both). The mismatch and efficiency of the Tx antenna will reduce the transmitted power introduced into the chamber, while the mismatch and efficiency of 
the Rx antenna will reduce the amount of power measured by the spectrum analyzer. Rewriting (7) to include the antenna and mismatch corrections, we now have:

$G_{\mathrm{CC}}[\mathrm{dB}]=10 \times \log _{10}\left[\frac{\left\langle P_{\mathrm{SA}}^{\mathrm{ref}}\right\rangle}{\left(P_{\mathrm{SG}}^{\mathrm{ref}}\right)} \times \frac{1}{e_{\mathrm{Tx}} \mathrm{e}_{\mathrm{Rx}} m_{\mathrm{Tx}} m_{\mathrm{Rx}}} \times \frac{1}{L_{\mathrm{tot}}}\right]$.

The various terms in (8) are defined as follows:

$e_{\mathrm{Tx}, \mathrm{Rx}}=$ antenna efficiencies (values are between 0 and 1 ),

$\mathrm{m}_{\mathrm{Tx}, \mathrm{Rx}}=$ impedance mismatches of the antennas connected to the SG and SA

(values are between 0 and 1 ).

\section{Step 3. Setting the Target Average-Total-Attenuation}

Once $G_{\mathrm{CC}}$ is determined, the variable attenuation is adjusted to the desired overall attenuation, or target average-total-attenuation, between the base station in the $\mathrm{AC}$ and the $\mathrm{RC}$. The target average-total-attenuation is set according to the process described in [1], [3] with the following change: the AC containing the RF PASS is replaced with the RC. The field uniformity at the plane containing the base station (or Tx antenna in Figure 32) is verified as described in [1]. The equation that describes the overall attenuation is

$P L_{\text {Target }}(\mathrm{dB})=G_{\mathrm{Tx}}(\mathrm{dB})-G_{\mathrm{CC}}(\mathrm{dB})+L_{V A R}(\mathrm{~dB})$.

In the case of the NFPA multipath test, the variable attenuation is set as needed to achieve the targeted average attenuation of $100 \mathrm{~dB}$. Thus, the average-total-attenuation from the plane (or table top) supporting the Tx antenna in the AC to the testing volume in the RC is set to $100 \mathrm{~dB}$. The target average-total-attenuation can then be verified by redoing steps 1 and 2 above, and recalculating the $P L_{\text {Target }}$, as in (9).

\section{Step 4. Carry Out Multipath Test}

After establishing the target attenuation condition, the SG and SA are disconnected from the AC and RC, and the Tx antenna and connecting cable are removed from the AC. The base station (e.g., laptop with base station software and USB-port connected antenna) is placed in the AC, where the Tx antenna was previously located. The Rx antenna and connecting cable is left in the RC during the testing. The bulkhead which connects the cable and Rx antenna is terminated with a $50 \Omega$ load. Figure 12 shows the setup with a base station and RF PASS unit.

A stepped movement of the paddle is used during the calibration process. However, during the multipath test, the paddles are turned continuously. As the paddle is moved, the multipath conditions change within the chamber, and thus the speed of rotation or movement can impact the measurement results. Based on [17] and laboratory testing during the development of this multipath test methodology, the paddle rotation is set for between 1 to 6 revolutions per minute. In the latest revision of the standard the revolutions per minute is set to 3 . 


\subsection{Results}

The intent of this test is to verify the RF PASS system performance while operating in a rich multipath environment. Thus, the pass/fail criteria should reflect intended operations when the system is deployed. There are two pass/fail criteria: 1) the reception of the motion alarm at the base station within 30 seconds of initiation by the RF PASS, or 2) the reception of the evacuation signal at the RF PASS within the 30 seconds of initiation at the base station. As discussed above, the paddle is moving while these signals are being sent, and thus the signal level changes due to multipath during the test. This is different than a point-to-point attenuation test, where the channel applies a fixed amount of attenuation through a single path between the base station and the RF PASS.

Figure 15 shows the motion alarm multipath results for units from four RF PASS manufacturers in histogram form, where repeat measurements ( $\mathrm{y}$ axis) were conducted for the RF PASS systems for various levels of introduced path loss ( $\mathrm{x}$ axis). The path loss threshold above which the RF PASS must operate (100 dB according to the Multipath Test in NFPA 1982) is indicated by the line at the $100 \mathrm{~dB}$ mark. The red color shows the introduced path loss value beyond which the pass criteria were not met. Due to the different frequencies and technologies of each manufacturer, failure occurs at different attenuation levels depending on the manufacturer.
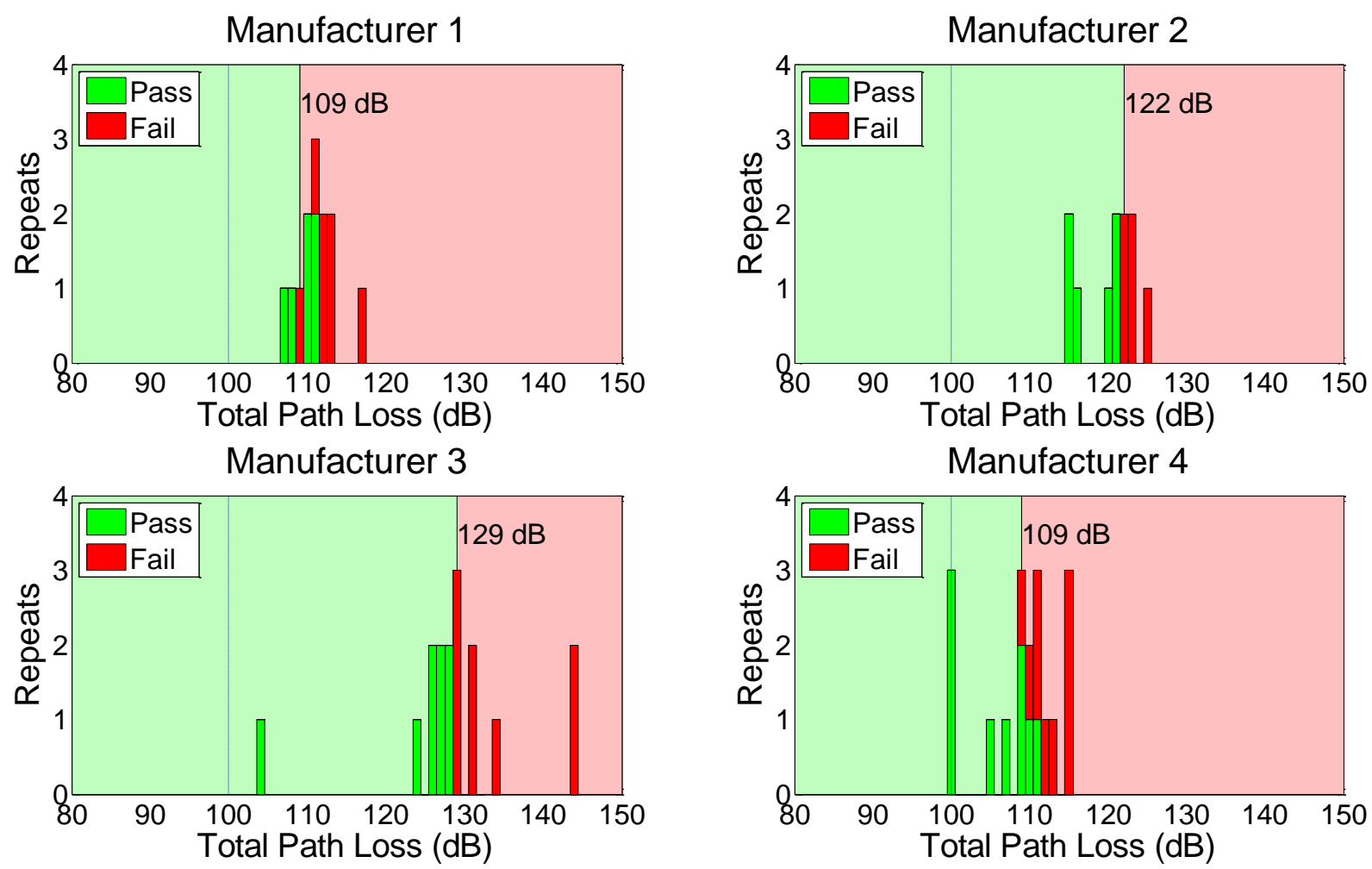

Figure 15. Motion alarm test results for all manufacturers of RF PASS for the Multipath Test.

Figure 16 shows the evacuation alarm multipath results for three manufacturers, again in histogram form. There are no results for manufacturer four because a human would have to be introduced 
into the chamber to send the evacuation alarm which would disturb the RF signals. Note that the RF PASS devices for each manufacturer successfully operate at the required attenuation levels. The point of failure as a function of introduced path loss is again indicated by the red color.
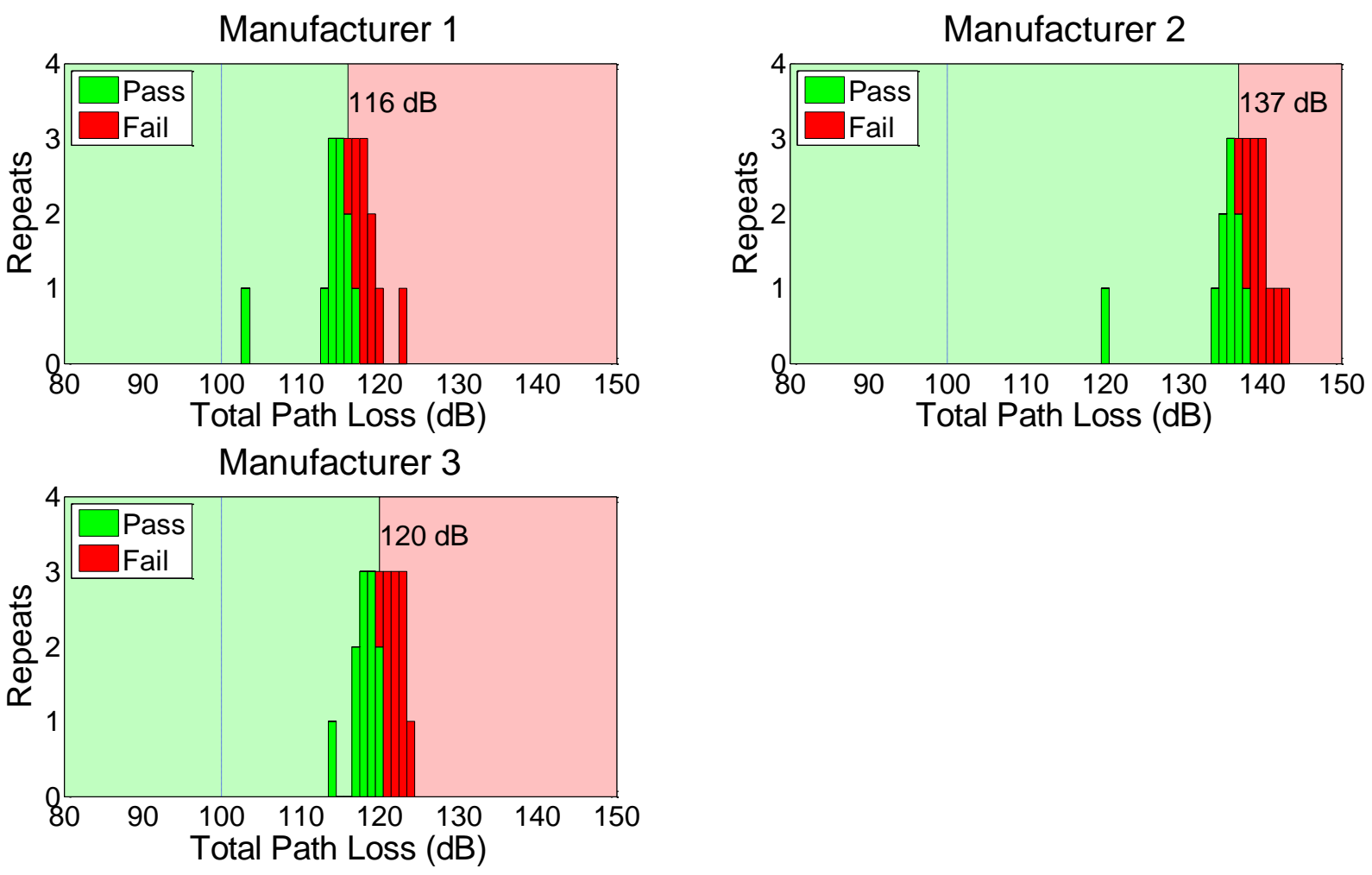

Figure 16. Evacuation alarm test results for all manufacturers of RF PASS for the Multipath Test. Results for manufacturer 4 were unable to be produced due to logistical reasons.

The results of the NIST tests of the four RF PASS systems indicate that these systems would all likely pass the Multipath Test given in NFPA 1982. However, NIST is not a certified NFPA test lab and official results may vary.

\section{Configuration Considerations for Point-Point, Multi- Hop, and Interference Testing}

The following sections discuss the setup and calibrations that were performed for three tests that involve anechoic chambers: the Point-to-Point, Multi-Hop and Interference (Low- and HighPower) Tests. These tests use a variety of equipment including, but not limited to, power combiners, spectrum analyzers, signal generators, anechoic chambers, and reverberation chambers. Many of the configurations discussed in Section 4 are applied in the Point-To-Point, Multi-Hop, and Interference testing described in Section 5. 


\subsection{Path Loss Measurements for Anechoic Chamber}

Path loss refers to the reduction in signal strength that occurs when a signal travels between the transmitter and receiver. If there is too much path loss between the firefighter and the base station, the distress signal might not activate in a timely manner or at all. The main focus of the following procedures is to determine an appropriate test setup to verify the RF PASS performance under path loss conditions ranging from below $100 \mathrm{~dB}$ to in excess of $250 \mathrm{~dB}$. The data presented in this section illustrate validation of the proposed setups.

We determined from results of the uniformity study of Section 2.4 that Patch D would be used for the $450 \mathrm{MHz}$ frequency range, Patch E would be used for the $900 \mathrm{MHz}$ frequency range, and that the helix antenna with the RF absorbing lens produced the most uniform electric field for the 2.4 $\mathrm{GHz}$ systems. For a path loss measurement in each band, these antenna types are mounted at the top interior of each chamber. Various antennas are placed on the test platform (at the bottom of the chamber) to transmit and receive signals from the top antenna.

In Section 4, we present an example where the anechoic chambers are configured and measured to determine path loss at $2.444 \mathrm{GHz}$. Several equations throughout this section are used to detail the verification procedure. The variables used in these equations are explained in Table 8.

Table 8. Variable descriptions for equation use.

\begin{tabular}{|c|c|}
\hline Term & Description \\
\hline$G_{A}$ & Gain of Antenna \\
\hline$G_{R x}$ & Gain from Receiving Antenna \\
\hline$G_{T x}$ & Cable Loss \\
\hline$L_{C}$ & Free Space Chamber Loss \\
\hline$L_{C o}$ & Free Space Chamber Loss of Anechoic Chamber \\
\hline$L_{F S C}$ & Power Combiner Loss \\
\hline$L_{F S C}^{A C}$ & Path Loss from Chamber 1 to 2 \\
\hline$L_{F S C}^{R C}$ & Pree Space Chamber Loss from Chamber 1 to 3 \\
\hline$L_{P C}$ & Path Loss from Chamber 2 to 3 \\
\hline$P L_{2}^{1}$ & Path Loss from Chamber 3 to 4 \\
\hline$P L_{3}^{1}$ & Measured Path Loss \\
\hline$P L_{3}^{2}$ & Total Path Loss \\
\hline$P L_{4}^{3}$ & Added Attenuation \\
\hline$P L A_{3}^{1}$ & Power Loss with Added Attenuation from Chamber 1 to 3 3 \\
\hline$P L_{m e a s}$ & Measured Value \\
\hline$P L_{t o t}$ & Power Amplifier \\
\hline AA & Signal Generator Input \\
\hline MV & \\
\hline PA & \\
\hline$S G I$ &
\end{tabular}




\subsubsection{Measurement of Cable Loss for an Individual Anechoic Chamber Setup}

When configuring the chambers to measure the total path loss $\left(P L_{t o t}\right)$ through an anechoic chamber, the first step is to measure the loss of the coaxial cables and other connectors that would contribute to $P L_{\text {tot }}$. The different components that need to be accounted for include four coaxial cables and a $20 \mathrm{~dB}$ attenuator. For the $2.4 \mathrm{GHz}$ set-up, the same RF absorbing foam lens shown in Figure 10(a) is also used throughout this section. Figure 17 below shows the set up.

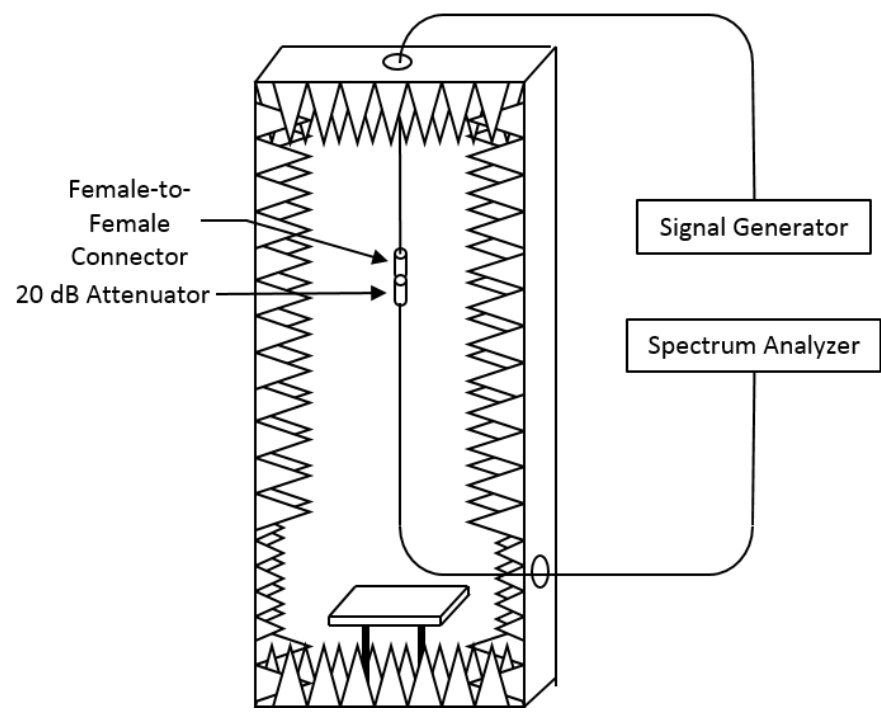

Figure 17. Set up for the path loss measurement calibration.

A $2.444 \mathrm{GHz}$ continuous wave (CW) signal with $0 \mathrm{dBm}$ amplitude was sent through this series of cables and connectors and was received by the spectrum analyzer to read $-25.4 \mathrm{dBm}$. The cables and connectors contributed to $5.4 \mathrm{~dB}$ of loss.

\subsubsection{Determining Antenna Type for RF PASS Testing Configuration}

For each AC setup, the target path loss values are determined by use of an antenna mounted to the top of the chamber (already determined for each frequency in Section 2.3.4) and a second calibration antenna placed on the measurement platform. An antenna with low path loss is ideal because it allows more flexibility in the path loss of other components. The goal of this section is to determine which calibration antenna will yield lowest path loss through an anechoic chamber. Once the cable loss was measured, the helix was mounted to the top of the chamber and different antennas were individually placed on the reference plane, as shown below in Figure 18. The goal was to determine which antenna created the lowest path loss while maintaining field uniformity at the table top. Measurements were taken for the path loss within the chamber and $L_{F S C}$ was then calculated according to (10) (see next page), where the variable descriptions are given in Table 8. The results of all trials are summarized in Table 9 and Table 10. The descriptions of each antenna can be found in Table 2 . 


$$
L_{F S C}=P L_{\text {meas }}-L_{C}+G_{R x}+G_{T x}
$$

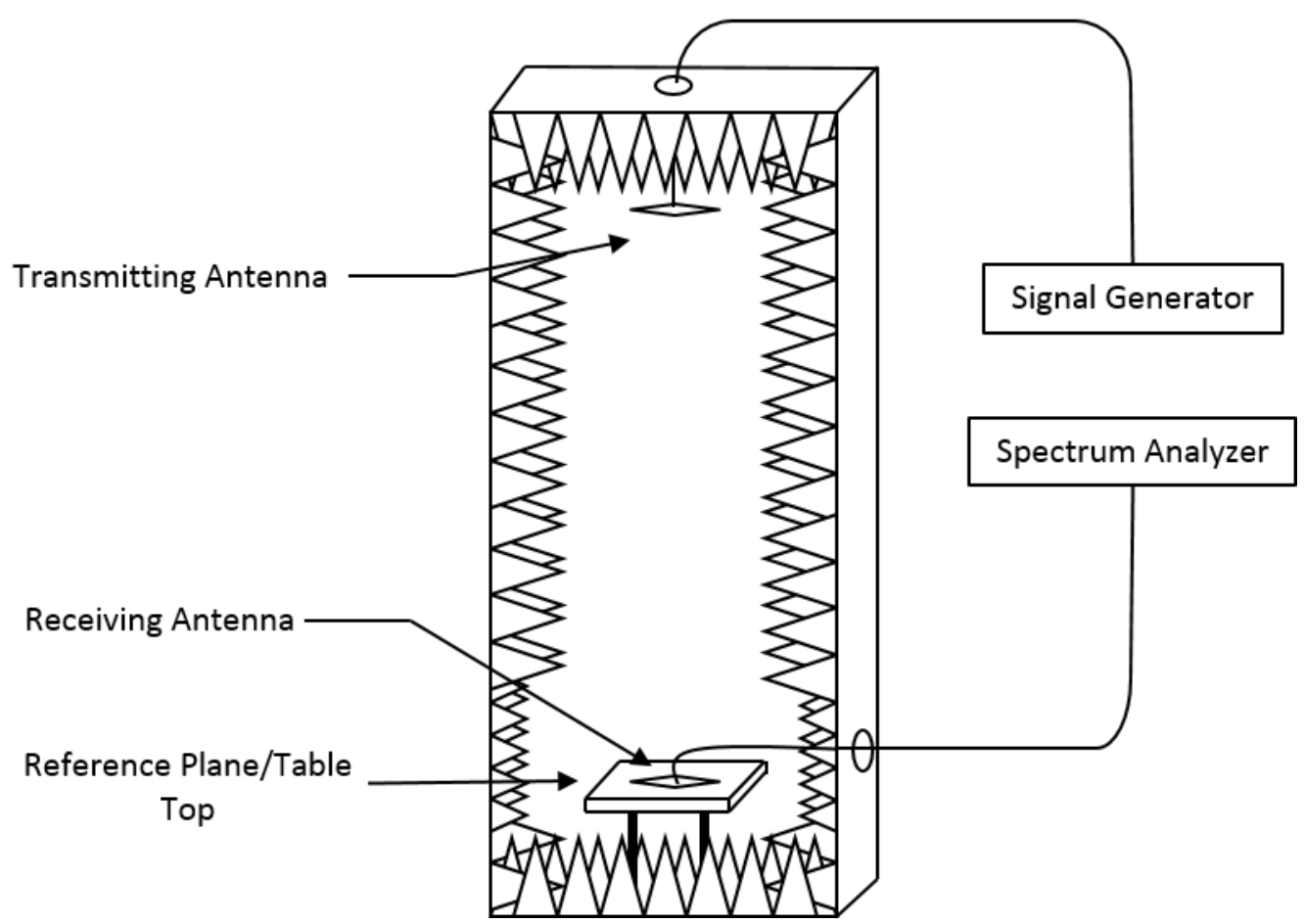

Figure 18. Setup for the path loss measurement data collection.

Table 9. Spectrum analyzer measurements for each $2.4 \mathrm{GHz}$ antenna and trial. The helix antenna is mounted as the transmitting antenna.

\begin{tabular}{|c|c|c|c|c|}
\hline & $\begin{array}{c}\text { Patch A } \\
(\mathbf{d B m})\end{array}$ & $\begin{array}{c}\text { Patch B } \\
(\mathbf{d B m})\end{array}$ & $\begin{array}{c}\text { Patch C } \\
(\mathbf{d B m})\end{array}$ & $\begin{array}{c}\text { Patch C With } \\
\text { Lens }(\mathbf{d B m})\end{array}$ \\
\hline Trial 1 & -38.7 & -37.6 & -32.1 & -30.3 \\
\hline Trial 2 & ------ & ----- & -32.2 & -30.3 \\
\hline Trial 3 & ------ & ------ & -32.1 & -30.3 \\
\hline
\end{tabular}

Table 10. $P L_{\text {tot }}$ for each $2.4 \mathrm{GHz}$ antenna and trial. The helix antenna is mounted as the transmitting antenna.

\begin{tabular}{|c|c|c|c|c|}
\hline & $\begin{array}{c}\text { Patch A } \\
\boldsymbol{L}_{\boldsymbol{F S C}}(\mathbf{d B})\end{array}$ & $\begin{array}{c}\text { Patch B } \\
\boldsymbol{L}_{\boldsymbol{F S C}}(\mathbf{d B})\end{array}$ & $\begin{array}{c}\text { Patch C } \\
\boldsymbol{L}_{\boldsymbol{F S C}}(\mathbf{d B})\end{array}$ & $\begin{array}{c}\text { Patch C } \\
\boldsymbol{L}_{\boldsymbol{F S C}} \text { With Lens }\left(\boldsymbol{P L}_{\boldsymbol{t o t}}\right)(\mathbf{d B})\end{array}$ \\
\hline Trial 1 & 51.3 & 50.2 & 43.7 & 41.9 \\
\hline Trial 2 & ----- & ----- & 43.8 & 41.9 \\
\hline Trial 3 & ----- & ----- & 43.7 & 41.9 \\
\hline
\end{tabular}

Patches A and B were the same type of antenna but with opposite polarizations. This should have caused one of the antennas to cross polarize with the transmitting antenna resulting in a higher path loss. Because of the small difference between these two antennas' results, we determined that patches $\mathrm{A}$ and $\mathrm{B}$ were poor quality antennas. Patch $\mathrm{C}$ had at least $5.5 \mathrm{~dB}$ less path loss compared to patch A or B which was favorable. For each trial, the receiving antenna was disconnected, reconnected, and repositioned on the platform. 
A helix antenna identical to the transmitting antenna was placed at the reference plane. With the $\mathrm{RF}$ absorbing lens in place, $P L_{\text {tot }}$ of the helix was found, see Table 11 . This loss was within $1 \mathrm{~dB}$ of patch $\mathrm{C}$, as shown in Table 10 .

This same process was repeated with a horn antenna (with specifications found in Table 2) on the reference plane. Because the antenna is linearly polarized, there were two orientations 90 degrees apart that were tested. Three trials were tested for each orientation. The antenna was disconnected, reconnected and placed on the platform for each trial. Results are shown in Table 11.

Table 11. $P L_{\text {tot }}$ measurements for three $2.4 \mathrm{GHz}$ antennas. The helix antenna is mounted as the transmitting antenna.

\begin{tabular}{|c|c|c|c|c|}
\hline & $\begin{array}{c}\text { Patch C } \\
\text { With Lens } L_{F S C}(\mathbf{d B})\end{array}$ & $\begin{array}{c}\text { Helix With } \\
\text { Lens } L_{F S C}(\mathbf{d B})\end{array}$ & $\begin{array}{c}\text { Horn With Lens } \\
\boldsymbol{L}_{F S C} \text { Position 1 } \\
(\mathbf{d B})\end{array}$ & $\begin{array}{c}\text { Horn With Lens } \\
\text { LFSC }_{F} \text { Position 2 (dB) }\end{array}$ \\
\hline $\begin{array}{c}\text { Trial } \\
1\end{array}$ & 41.9 & 42.8 & 45.7 & 42.7 \\
\hline $\begin{array}{c}\text { Trial } \\
2\end{array}$ & 41.9 & -------- & 45.7 & 43.0 \\
\hline $\begin{array}{c}\text { Trial } \\
3\end{array}$ & 41.9 & ------- & 45.9 & 42.8 \\
\hline
\end{tabular}

The results showed that patch $\mathrm{C}$ yielded the lowest path loss. $41.9 \mathrm{~dB}$ is the $L_{F S C}$ within the anechoic chamber before taking out the $10 \mathrm{~dB}$ gain from the helix. Therefore $P L_{\text {tot }}$ within the chamber is $31.9 \mathrm{~dB}$.

\subsubsection{Path Loss between Two Anechoic Chambers}

The purpose of this section is to determine the path loss from test platform to test platform through two anechoic chambers. This value is then used to determine how much added attenuation will be needed to achieve $100 \mathrm{~dB}$ path loss between test platforms. A value of $100 \mathrm{~dB}$ of path loss between devices located on test platforms in two anechoic chambers is required for several test methods, including the point-to-point, coexistence, multipath, and multi-hop methods.

For some tests, a power combiner is used between chambers. For this, the insertion and isolation values are determined. A basic power combiner setup is shown in Figure 19. One port was terminated with a $50 \Omega$ load while the other two were connected to a signal generator and spectrum analyzer to determine the insertion and isolation loss. The insertion loss was $3.5 \mathrm{~dB}$ and the isolation loss was $24.2 \mathrm{~dB}$. 


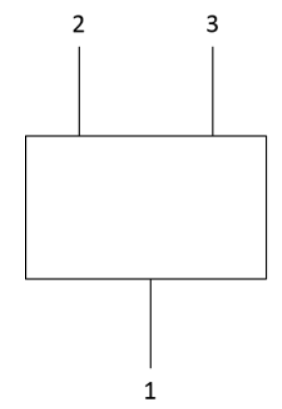

Figure 19. Power combiner and labeled ports.

\subsubsection{Chambers 1 and 2}

Below are the experimental results for path loss between two anechoic chambers; we again used the $2.4 \mathrm{GHz}$ frequency band as an example. Two antennas were used in the following experiment for comparison. As decided in Section 2.4, the helix will be the top antenna for any subsequent tests. A helix and a horn were used to send the signal from the identical antenna in the reference plane in chamber 1 to the reference plane in chamber 2. This reference plane is shown in Figure 18; that is, a signal traveled from a horn antenna to a horn antenna or a helix antenna to a helix antenna through the helix antennas at the top of the chambers. In the setup shown in Figure 20, the antenna on the reference plane in chamber 1 transmits, and the antenna on the reference plane in chamber 2 receives. Because the horn is linearly polarized, two orientations with 90 degree rotations were measured. The cable loss was measured to be $7.5 \mathrm{~dB}$. Figure 20 shows the setup. Table 12 shows the results collected by the spectrum analyzer and Table 13 shows $P L_{\text {tot }}$.

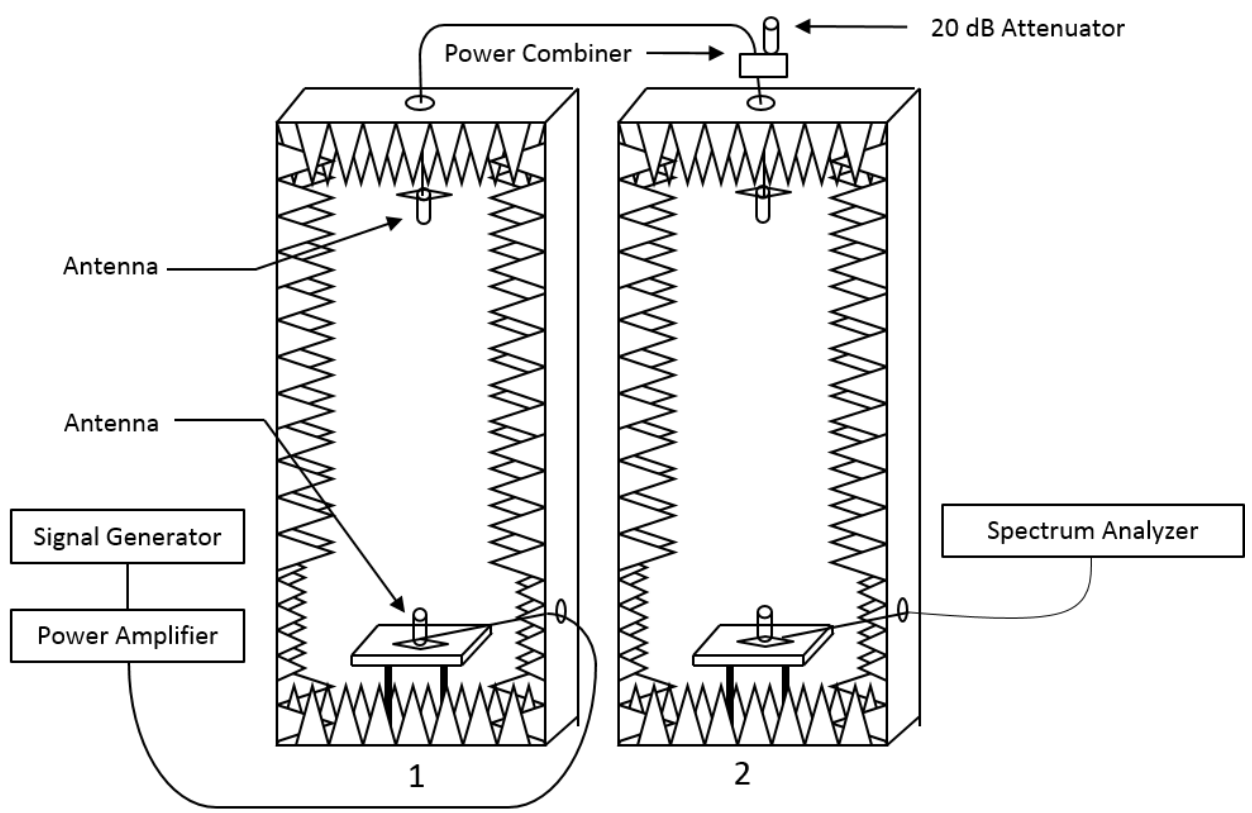

Figure 20. Setup for chambers 1 and 2 with the power combiner. 
Table 12. Spectrum analyzer measured values for system calibration from chamber 1 to 2 with power combiner. The helix antenna is mounted to the top of the chamber as the transmitting antenna.

\begin{tabular}{|c|c|}
\hline Antenna on Reference Plane & Measured Value \\
\hline Helix & $-55.0 \mathrm{dBm}$ \\
\hline Horn Position 1 & $-65.1 \mathrm{dBm}$ \\
\hline Horn Position 2 & $-64.8 \mathrm{dBm}$ \\
\hline
\end{tabular}

We then calculated $P L_{\text {tot }}$ for each antenna, using [3]:

$$
P L_{t o t}=\operatorname{abs}\left(M V-(S G I+P A)-\left(G_{T x}\right)-\left(G_{R x}\right)+L_{C}\right) .
$$

See Table 8 for the definition of the variables. For example, with the $2.4 \mathrm{GHz}$ helix antenna on the reference plane in both chambers, $P L_{t o t}$ can be calculated as follows:

$$
P L_{\text {tot }}=-55.0 \mathrm{dBm}-(-50 \mathrm{dBm}+55 \mathrm{~dB})-10 \mathrm{dBi}-10 \mathrm{dBi}+7.5 \mathrm{~dB}=72.5 \mathrm{~dB} .
$$

Table 13. $P L_{\text {tot }}$ of receiving antennas at $2.4 \mathrm{GHz}$, the helix is the transmitting antenna.

\begin{tabular}{|c|c|}
\hline Antenna on Reference Plane & $\boldsymbol{P L}_{\text {tot }}$ \\
\hline Helix & $72.5 \mathrm{~dB}$ \\
\hline Horn Position One & $74.6 \mathrm{~dB}$ \\
\hline Horn Position Two & $74.3 \mathrm{~dB}$ \\
\hline
\end{tabular}

These data show that the helix and horn provide similar values of path loss.

\subsubsection{Chambers 1 and 2 with Added Attenuation}

The setup for the point-to-point test between chamber 1 and 2 with added attenuation differs from the other experiment only by adding a variable attenuator in series between chambers to achieve a value of path loss from test platform to test platform that is as close to $100 \mathrm{~dB}$ as possible. The input consisted of a $-50 \mathrm{dBm}$ signal and a $55 \mathrm{~dB}$ gain amplifier. The measurement results are shown in Table 14. $P L_{\text {tot }}$ was then calculated for each antenna with (8) and the results are listed in Table 15.

Table 14. Antennas and their respective added attenuation and measured values for $2.4 \mathrm{GHz}$.

\begin{tabular}{|c|c|c|}
\hline Antenna on Reference Plane & Measured Value & Added Attenuation \\
\hline Helix & $-82.3 \mathrm{dBm}$ & $27 \mathrm{~dB}$ \\
\hline Horn Position 1 & $-90.0 \mathrm{dBm}$ & $24 \mathrm{~dB}$ \\
\hline Horn Position 2 & $-89.5 \mathrm{dBm}$ & $22 \mathrm{~dB}$ \\
\hline
\end{tabular}

Table 15. Antennas and respective $P L_{t o t}$ with added attenuation for $2.4 \mathrm{GHz}$.

\begin{tabular}{|c|c|}
\hline Antenna on Reference Plane & $\boldsymbol{P} \boldsymbol{L}_{\text {tot }}$ \\
\hline Helix & $99.8 \mathrm{~dB}$ \\
\hline Horn Position One & $99.5 \mathrm{~dB}$ \\
\hline Horn Position Two & $99.0 \mathrm{~dB}$ \\
\hline
\end{tabular}




\subsubsection{RF PASS Performance between Two Anechoic Chambers}

After attenuation was added to configure the chambers for $100 \mathrm{~dB}$ path loss, the devices were placed on the test platforms in the anechoic chambers. The setup shown in Figure 21 is similar to that of the point-to-point RF test in [1]. The difference in the following setup from the existing point-to-point test lies in the power combiner and $20 \mathrm{~dB}$ attenuator that allow the system to be connected to other chambers for the multi-hop test.

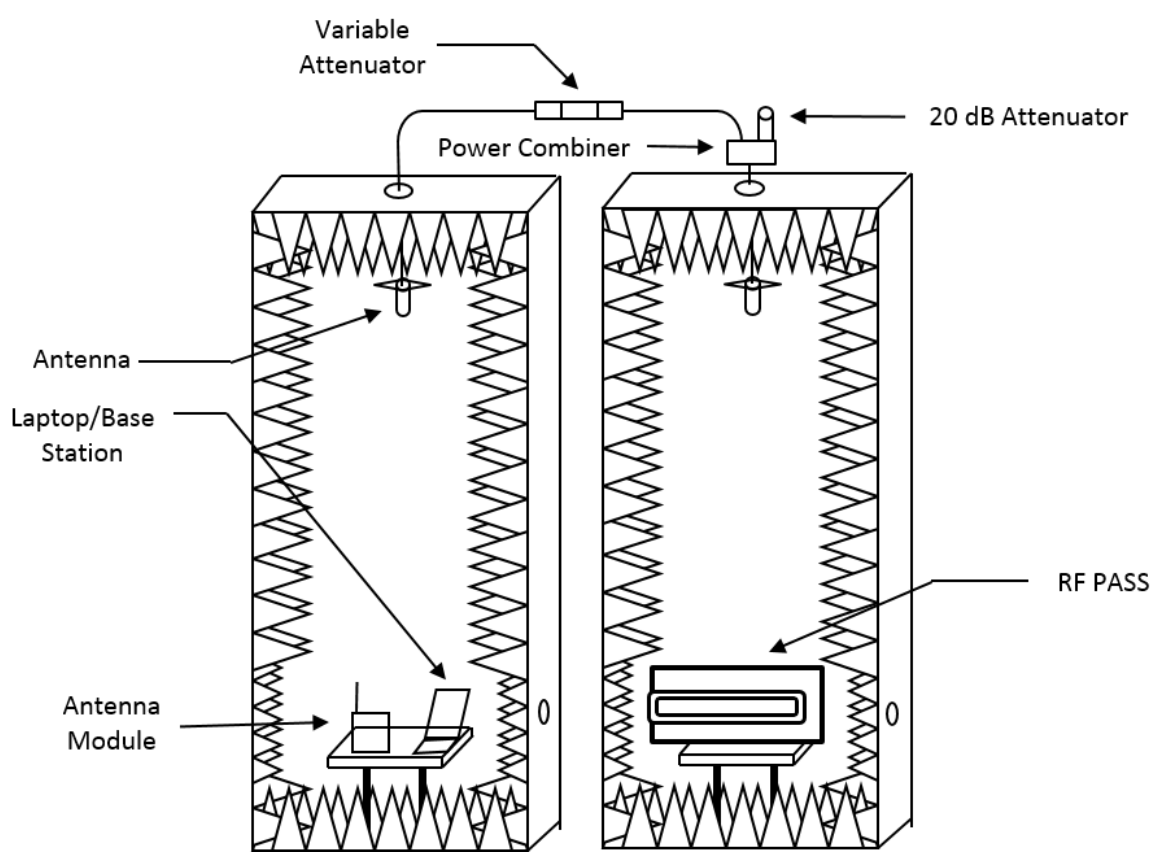

Figure 21. RF-PASS-device-to-base-station configuration for two anechoic chambers.

\subsection{Reverberation Chamber Calibration and RF PASS Stirrer Speed Dependency}

One use of a reverberation chamber is in the multipath test, as previously discussed. Another use for this type of chamber is in the multi-hop test which will be discussed in a later section. The calibration for the cables and components throughout the reverberation chamber is similar to that described in Section 3. However, for the multipath test, the calibration of the reverberation chamber is performed by connecting it to an anechoic chamber, whereas for the multi-hop test the path loss of the reverberation chamber alone is found. Then, in the four chamber multi-hop test, each individual chamber is added into the total system's path loss. We must verify that the stirrer speed of the reverberation chamber does not alter the time it takes for the base station to receive the motion alarm, nor the RF PASS to receive the evacuation alarm. While the exact values may change based on reverberation size, reflectivity, etc., the following procedure should be performed to determine the effects of stirrer speed on the alarm timing.

\subsubsection{Reverberation Chamber Calibration}

The setup for the reverberation chamber calibration is shown in Figure 22. 


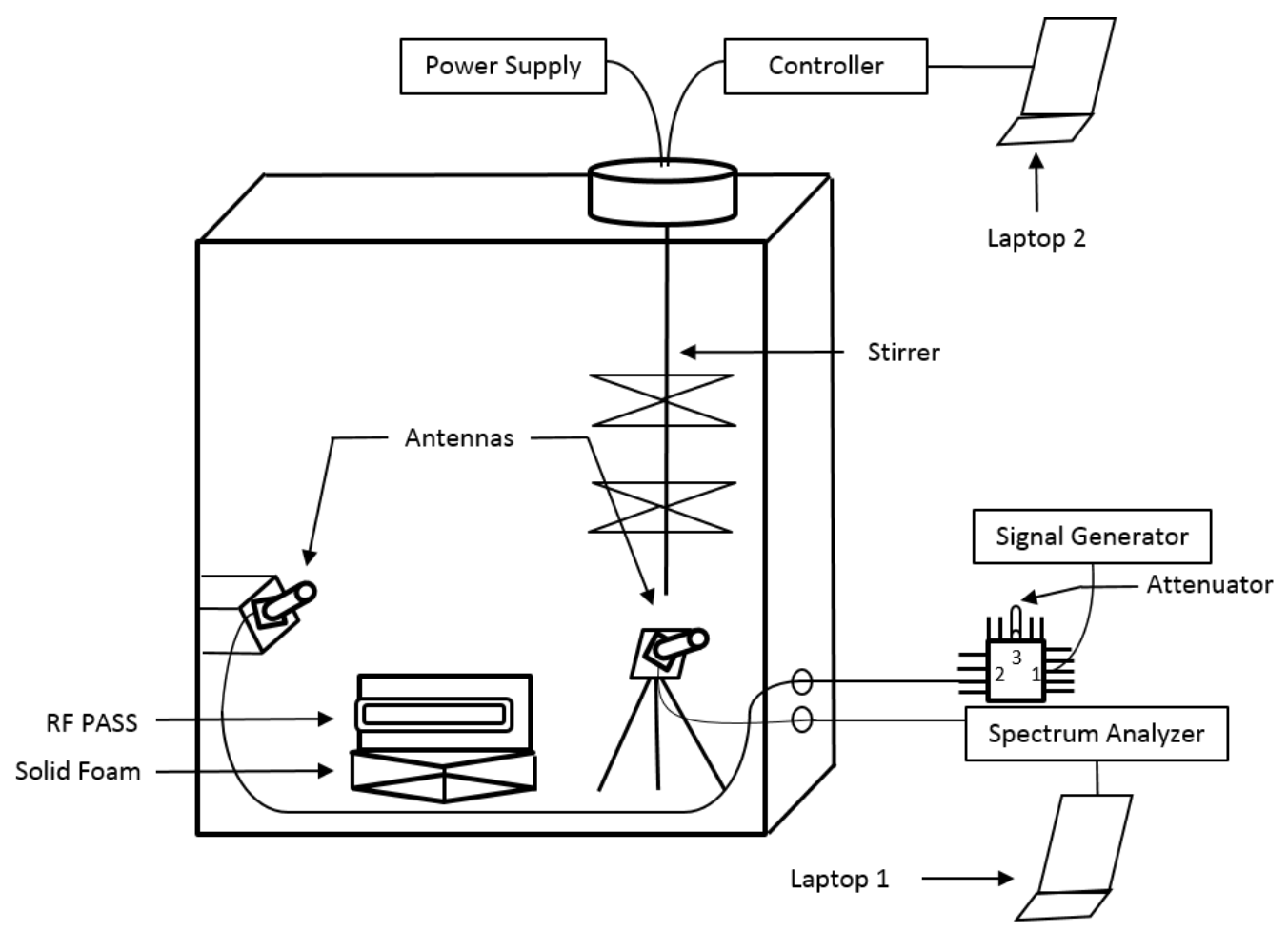

Figure 22. Calibration for reverberation chamber.

Laptop 1 is connected to the spectrum analyzer and collects/saves data at a user-specified interval. Laptop 2 controls the motor that sets the rate at which the paddle rotates. Two laptops were used only because each contained different software. One laptop with all necessary software would perform the same tasks as stated above. For the multipath and multi-hop tests, the paddle is rotated at 0.2 revolutions per minute and data are collected every three seconds. In this way, power measurements of 100 paddle positions over 360 degrees are collected and averaged to determine the path loss of the chamber.

\subsubsection{Stirrer Speed Dependency 2.4 GHz Manufacturer 3 RF PASS}

We performed the calibration illustrated in Figure 22 using the helix antenna. The average path loss within the reverberation chamber for this system was $45.2 \mathrm{~dB}$, where the path loss was found as in Section 3.5 equation (6), but with $L_{\mathrm{tot}}[\mathrm{dB}]=L_{\mathrm{RC}}^{\mathrm{SG}}[\mathrm{dB}]+L_{\mathrm{RC}}^{\mathrm{SA}}[\mathrm{dB}]+L_{\mathrm{RC}}^{\mathrm{Tx}}[\mathrm{dB}]+L_{\mathrm{RC}}^{\mathrm{Rx}}[\mathrm{dB}]$. At $2.4 \mathrm{GHz}$, there was $3.8 \mathrm{~dB}$ loss from the cables and the input was $-20 \mathrm{dBm}$ resulting in $21.4 \mathrm{~dB}$ average path loss in the reverberation chamber. The free space loss in the reverberation chamber will be taken as $21.4 \mathrm{~dB}$.

The goal was to achieve $100 \mathrm{~dB}$ average path loss between the reverberation and anechoic chambers. The path loss from within the anechoic chamber was $31.9 \mathrm{~dB}$ as found in the previous section, and the path loss from within the reverberation chamber was $21.4 \mathrm{~dB}$. To achieve $100 \mathrm{~dB}$ average path loss, $46 \mathrm{~dB}$ attenuation was added in line between the two chambers.

Figure 23 shows the setup to determine how the stirrer speed affects the connection between the base station and RF PASS with the added attenuation in line. 


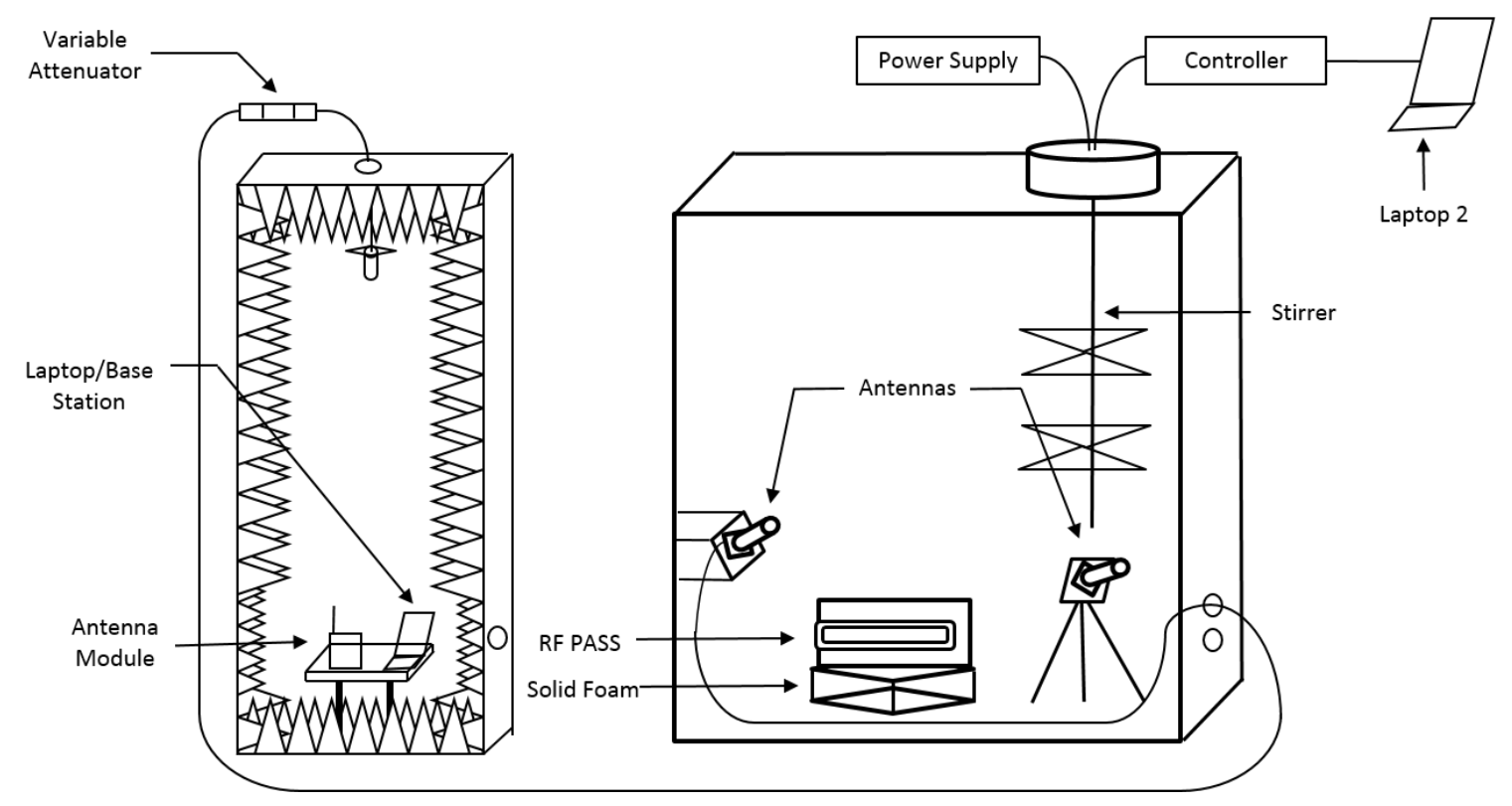

Figure 23. Two segments of the Multi-Hop test between reverberation chamber and anechoic chamber.

For all RF PASS manufacturers, the RF PASS was placed inside the chamber on a piece of solid foam to keep the device at least one wavelength's distance away from all chamber surfaces (including the floor). A dielectric rod was attached to the device to keep the RF PASS device moving to ensure the motion alarm did not go off until desired. The amount of time it took for the base station to receive the motion alarm from the RF PASS device was recorded in Table 16 for different paddle rotation speeds.

Table 16. Time recorded for base station to receive motion alarm from RF PASS device with $46 \mathrm{~dB}$ added attenuation in line.

\begin{tabular}{|c|c|}
\hline Revolutions/Minute & Receive Time (sec) \\
\hline 1 & 11.56 \\
\hline 2 & 11.63 \\
\hline 3 & 11.76 \\
\hline 4 & 11.48 \\
\hline 5 & 11.22 \\
\hline 6 & 11.28 \\
\hline
\end{tabular}

From these results, we can conclude that the speed at which the paddle rotates within a range from one to six revolutions per minute does not significantly affect the time it takes to send the motion alarm from the RF PASS.

\subsubsection{Stirrer Speed Dependency 900 MHz Manufacturer 1 RF PASS}

We repeated the process in Figure 18, using patch $\mathrm{E}$ as the receiving and transmitting antennas. The path loss within the anechoic chamber was $32.8 \mathrm{~dB}$. A $9 \mathrm{~dB}$ antenna gain was subtracted from this number to arrive at $23.8 \mathrm{~dB}$ path loss within the anechoic chamber. The same process shown in Figure 23 was repeated to find the path loss within the reverberation chamber with the $900 \mathrm{MHz}$ Manufacturer 1 device in place. At $900 \mathrm{MHz}$, there was $18.5 \mathrm{~dB}$ path loss within the reverberation 
chamber. This yielded $42.3 \mathrm{~dB}$ path loss between the anechoic chamber and reverberation chamber, so $58 \mathrm{~dB}$ attenuation was added to achieve $100 \mathrm{~dB}$ total path loss. For each trial, the cables were disconnected to test for proper isolation by ensuring the base station and portable RF PASS device could not connect wirelessly before proceeding with the test. The results are summarized below in Table 17.

Table 17. Stirrer speed dependency results for $900 \mathrm{MHz}$ Manufacturer 1 PASS systems showing time until motion alarm was received.

\begin{tabular}{|c|c|c|}
\hline Revolutions/Minute & Trial One Results (sec) & Trial Two Results (sec) \\
\hline 1 & 9.30 & 8.64 \\
\hline 2 & 8.47 & 9.25 \\
\hline 3 & 5.98 & 11.61 \\
\hline 4 & 12.70 & 10.55 \\
\hline 5 & 8.35 & 8.61 \\
\hline 6 & 9.80 & 7.30 \\
\hline
\end{tabular}

Trial one shows that there is little consistency in the time it takes for the motion alarm signal to reach the base station. Comparing trial one and trial two shows there is little dependency on the stirrer speed.

\subsubsection{Stirrer Speed Dependency $900 \mathrm{MHz}$ Manufacturer 2 RF PASS}

The RF PASS of Manufacturer 2 was also a $900 \mathrm{MHz}$ system so the same antennas (patch E) were used. The same $23.8 \mathrm{~dB}$ path loss within the anechoic chamber was used to determine the added attenuation needed to achieve $100 \mathrm{~dB}$ path loss. The RF PASS device of Manufacturer 2 was placed in the reverberation chamber and the process in Figure 23 was repeated to yield $17.8 \mathrm{~dB}$ path loss within the reverberation chamber. For this system, the two chambers had to be physically separated to achieve proper shielding. An additional cable with $3 \mathrm{~dB}$ loss was added to the system. Without this cable, we would have needed to add $58 \mathrm{~dB}$ to the system; with this cable, only $55 \mathrm{~dB}$ was added in line, to achieve $100 \mathrm{~dB}$ total path loss. After each test, without opening the reverberation chamber, the cables were disconnected, the base station motion alarm acknowledgement was reset, and the system was tested for proper isolation by ensuring the motion alarm was not received by the base station. The results can be seen in Table 18.

Table 18. Results for stirrer speed dependency with Manufacturer 2 showing time until motion alarm was received.

\begin{tabular}{|c|c|}
\hline Revolutions/Minute & Trial One Results $(\mathrm{sec})$ \\
\hline 1 & 1.09 \\
\hline 2 & 0.55 \\
\hline 3 & 0.70 \\
\hline 4 & 0.68 \\
\hline 5 & 0.63 \\
\hline 6 & 0.86 \\
\hline
\end{tabular}

For each case the motion alarm was received almost instantly. 1 revolution per minute varied more than the other trials merely because the timer was stopped a little late. As explained above, there was little dependency on stirrer speed for any of the three manufacturers. 


\subsection{Path Loss across Four Chambers}

This procedure determines how much added attenuation is needed when all four chambers are connected in series for the multi-path test. The path loss between each chamber was calculated by factoring in individual connections, cables, and free space losses of the particular chamber. The same RF absorbing lens used in Figure 10(a) for the $2.4 \mathrm{GHz}$ tests was used in each of the four chambers shown in Figure 24, although it is not shown.

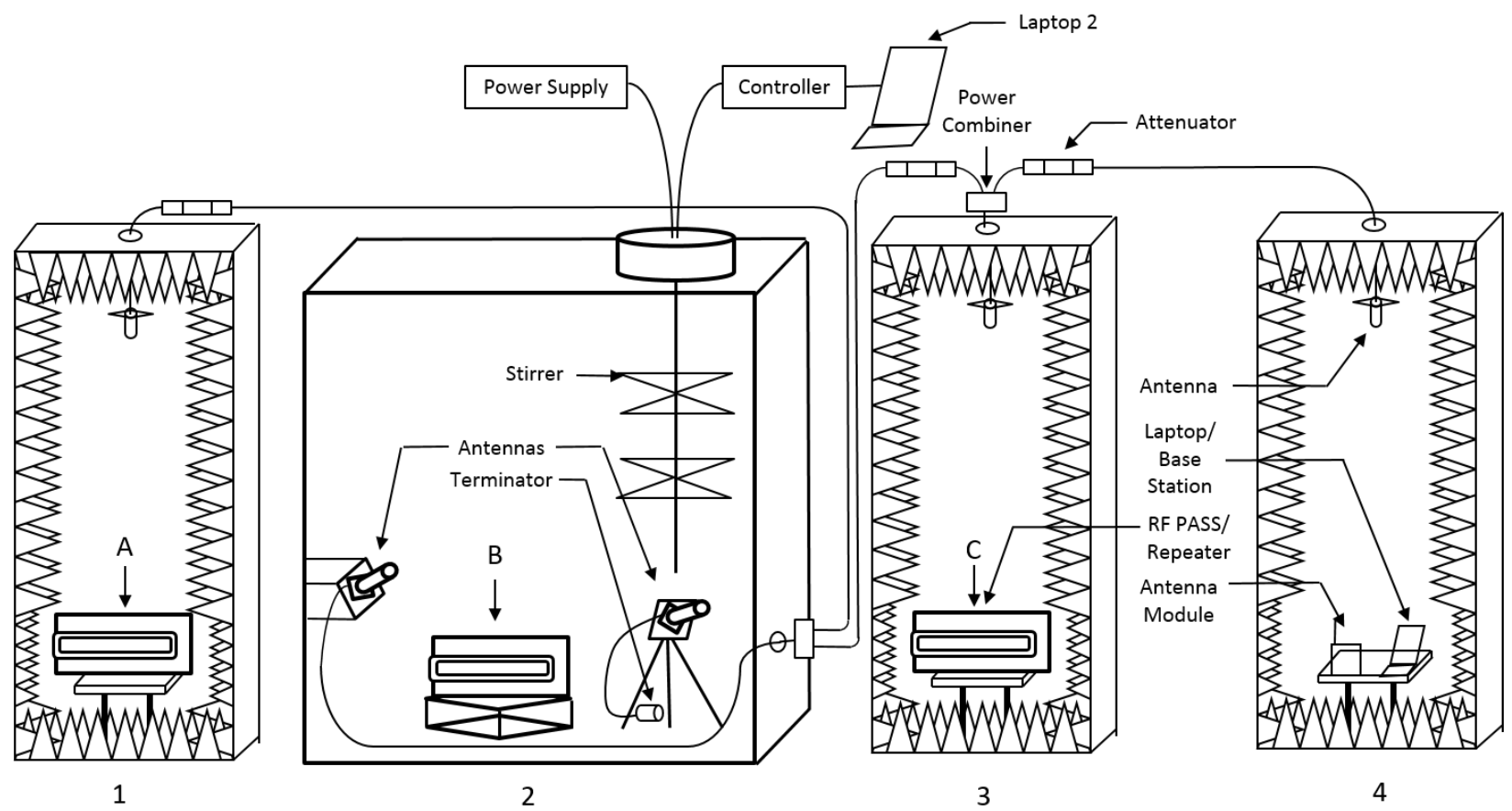

Figure 24. Path loss set up for four chambers.

\subsubsection{Path Loss Calculations}

For the Multi-hop test, the total path loss from the test platform in Chamber 1 through the reverberation chamber and through two additional anechoic chambers should be $260 \mathrm{~dB}$. Because this is difficult to measure directly, we found this value by adding together the path loss between pairs of chambers. $P L_{\text {tot }}$ was calculated between different chambers and is shown below in (12), (13) and (14) for the $2.4 \mathrm{GHz}$ system. Variable descriptions can be found in Table 8.

$$
\begin{gathered}
P L_{2}^{1}=L_{F S C}^{A C}-G_{A}+3 L_{C}+2 \mathrm{~L}_{C o}+L_{P C}+L_{F S C}^{R C}=61.35 \mathrm{~dB} \\
P L_{3}^{2}=L_{F S C}^{R C}+3 L_{C}+2 L_{P C}+2 L_{C o}+L_{F S C}^{A C}-G_{A}=63.6 \mathrm{~dB} \\
P L_{4}^{3}=2\left(L_{F S C}^{A C}-G_{A}\right)+3 L_{C}+4 L_{C o}+L_{P C}=69.65 \mathrm{~dB}
\end{gathered}
$$

The superscript refers to the first chamber and the subscript to the second chamber in each chamber pair, as shown in Fig. 24. For all of these calculations, $31.9 \mathrm{~dB}$ was used as the path loss value for each anechoic chamber (including antenna gain), as found in Section 4.1.2. This value was based on a measurement obtained with Patch $\mathrm{C}$ in a single chamber. However, when measuring the path 
loss between two chambers, a horn was used. To fix this discrepancy, the $L_{F S C}$ within the anechoic chamber will be taken as $44.3 \mathrm{~dB}$, the average of the two free-space loss horn position values found in Table 11. Using this value and the same equations above, we calculate the new path loss values between chambers. The corrected results are then

$$
\begin{gathered}
P L_{2}^{1}=63.75 \mathrm{~dB} \\
P L_{3}^{2}=66 \mathrm{~dB} \\
P L_{4}^{3}=74.45 \mathrm{~dB}
\end{gathered}
$$

These values, as expected, resemble their measured counterparts in Section 4.1.2. The measured values for the $P L_{4}^{3}$ (Section 4.1.2) obtained with the horn in position one was $74.6 \mathrm{~dB}$, and in position two was $74.3 \mathrm{~dB}$. These are compared with the calculated $P L_{4}^{3}$ value stated above at 74.45 $\mathrm{dB}$ with a horn position in between the first and second.

\section{Point-to-Point, Multi-Hop and Interference Testing}

In this section, we describe the Point-to-Point, Multi-Hop, and Interference Test methods using pieces of the calibrations discussed in Section 4.

\subsection{Point-to-Point Motion and Evacuation Alarm Test}

\subsubsection{Description}

The Point to-Point Motion Alarm and Evacuation Tests are designed to create a channel with a known path loss between the RF PASS device and its base station. After conducting a set of field tests and analyzing the data for various types of buildings encountered by firefighters, a value of $100 \mathrm{~dB}$ path loss between the portable unit and the base station was deemed to be a representative amount of attenuation for which point-to-point communication should be reliable [4, 18]. By isolating the RF PASS device from the base station, determining the path loss between chambers, and then adding the remaining attenuation to the channel, we were able to test the systems at the $100 \mathrm{~dB}$ mark. If a motion alarm was received by the base station within 30 seconds, the system passed. If the evacuation test was received by the RF PASS within 30 seconds, the system passed. An example setup based on a $900 \mathrm{MHz}$ system is presented, followed by NIST results for four manufacturers: e.g., one at $450 \mathrm{MHz}$, two at $900 \mathrm{MHz}$ and one at $2.4 \mathrm{GHz}$.

For the setup configuration, first the cable loss was measured with a signal generator and spectrum analyzer (see Section 4.1.1). The input on the signal generator was set to $0 \mathrm{dBm}$, the spectrum analyzer read $-2.1 \mathrm{dBm}$. This leads to a cable path loss of $2.1 \mathrm{~dB}$. Next, the $P L_{\text {tot }}$ within the anechoic chambers was measured with $0 \mathrm{~dB}$ external attenuation (see Section 4.1.3.1). 
To configure the chambers to provide $100 \mathrm{~dB}$ between RF PASS and the portable base station, the calibration in Section 4.1.3.2 was performed. Figure 25 shows the calibration setup.

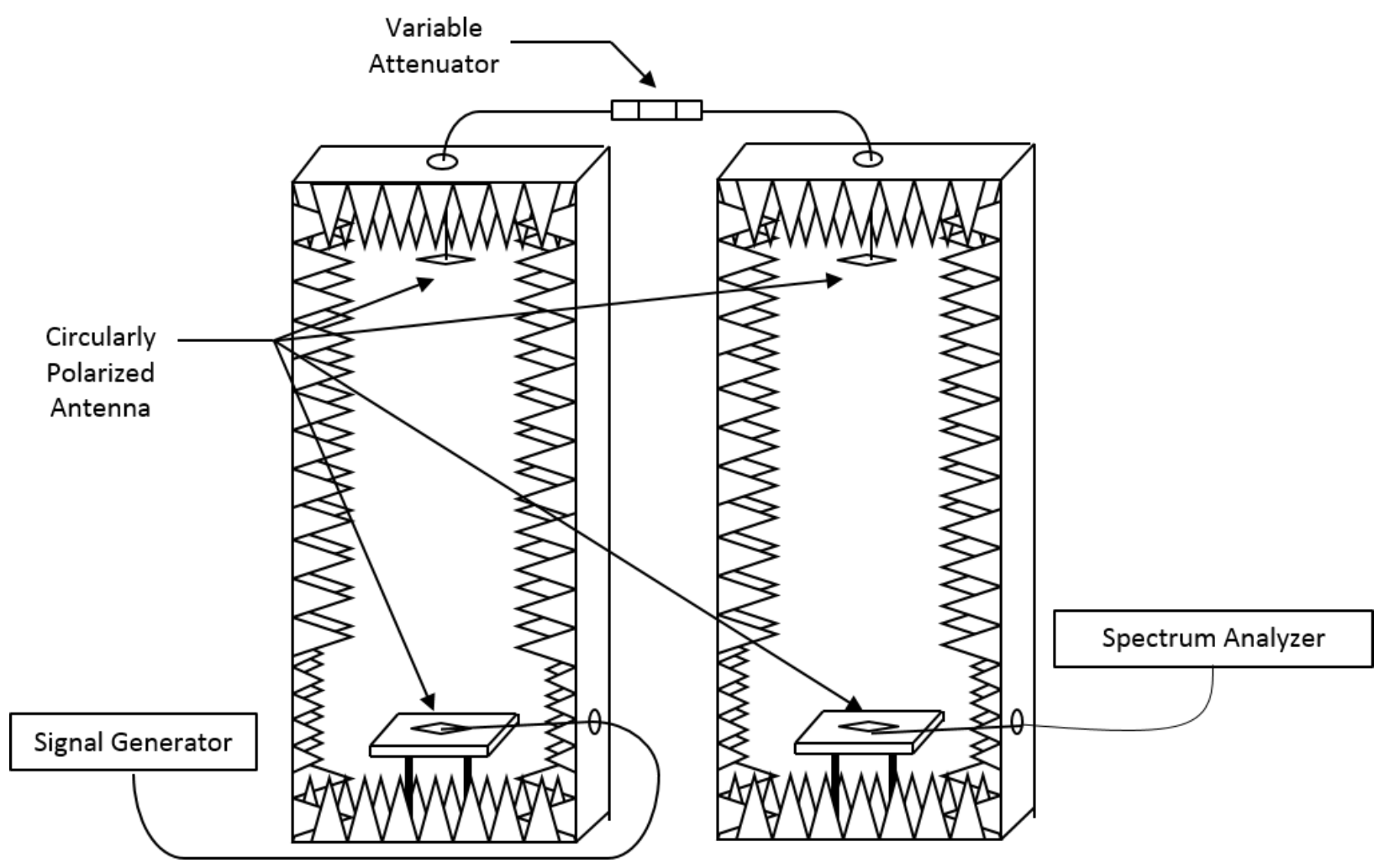

Figure 25. Setup for the Point-to-Point motion and Evacuation Alarm test calibration with circularly polarized receiving and transmitting antennas. The spectrum analyzer was configured to sweep from 900 $\mathrm{MHz}$ to $930 \mathrm{MHz}$. The signal generator was set to $0 \mathrm{dBm}$.

As an example, at $900 \mathrm{MHz}$, the spectrum analyzer read $-33.6 \mathrm{dBm}$, so the path loss of cables = $33.6 \mathrm{~dB}$. Using (15) we can obtain the total path loss from the location of the PASS device to the location of the base station. The antenna gain was $9 \mathrm{dBi}$ (see Table 2, Section 2.2).

$$
P L_{\text {tot }}=M V-L_{C}+2 G_{A}=49.5 \mathrm{~dB} \text {. }
$$

This leads to a value of roughly $51 \mathrm{~dB}$ external attenuation needed to generate a total $100 \mathrm{~dB}$ path loss between the PASS device and base station. 
For the point-to-point test, we achieved the desired path loss using external added attenuation, as described in Section 4.1.3.2. Then the PASS device was placed on the test platform in one chamber and its base station was placed on the test platform in the other chamber. A connection was established between the two. The doors were closed and a timer was set immediately after the audible motion alarm sounded on the PASS device. If the base station received the motion alarm from the PASS device within 30 seconds of the alarm being sent, the device passed the test. The test setup is shown in Figure 26.

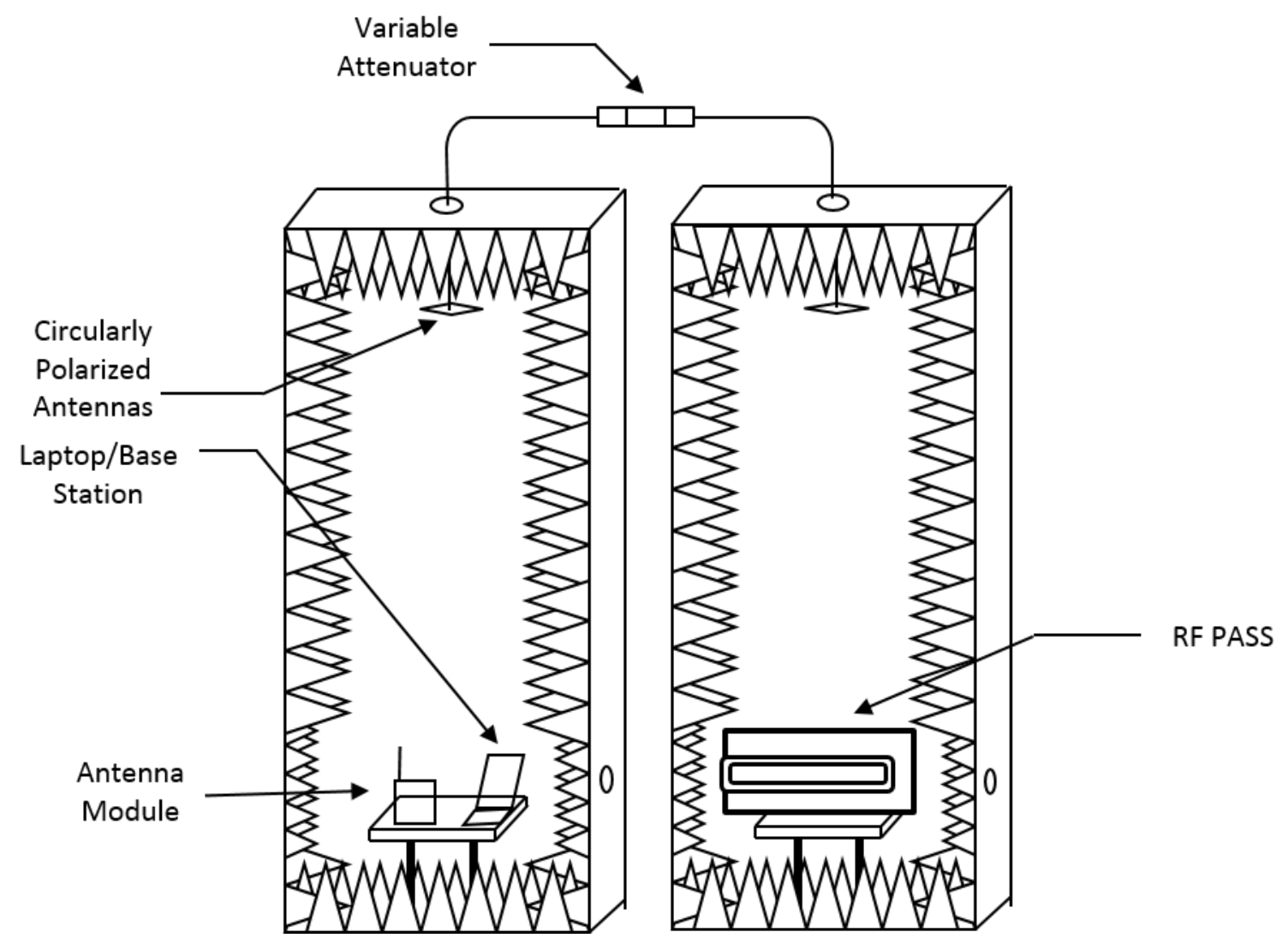

Figure 26. Set up for the Point-to-Point Motion and Evacuation Alarm tests with use of circularly polarized antennas. The evacuation alarm test uses the setup as shown, with an additional dielectric rod inserted into the RF PASS chamber and attached to the portable device. The operator moves the dielectric rod to keep the motion alarm from activating.

\subsubsection{NIST Tests: Point-to-Point Motion Alarm Results}

Figure 27 through Figure 30 show the results of the Point-to-Point Motion Alarm test for four different manufacturers of RF PASS. Positions one and two use the portable unit vertical and rotated at zero and 90 degrees. Positions three and four are horizontal and rotated at zero and 90 degrees. The line at $100 \mathrm{~dB}$ shows the threshold path loss value. Several repeats were conducted for each test. 
Manufacturer 1 Point-to-Point Motion Alarm
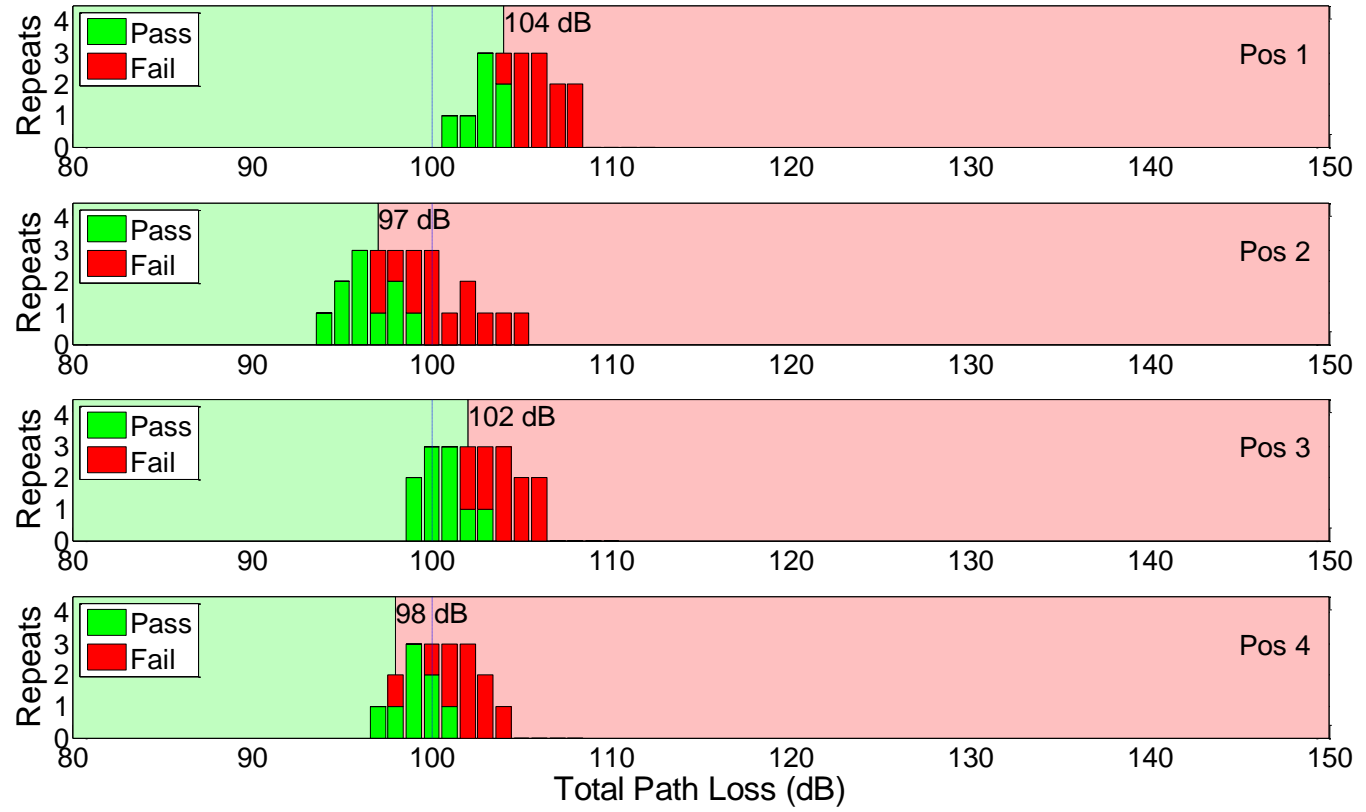

Figure 27. Point-to-Point Motion Alarm test for Manufacturer 1.
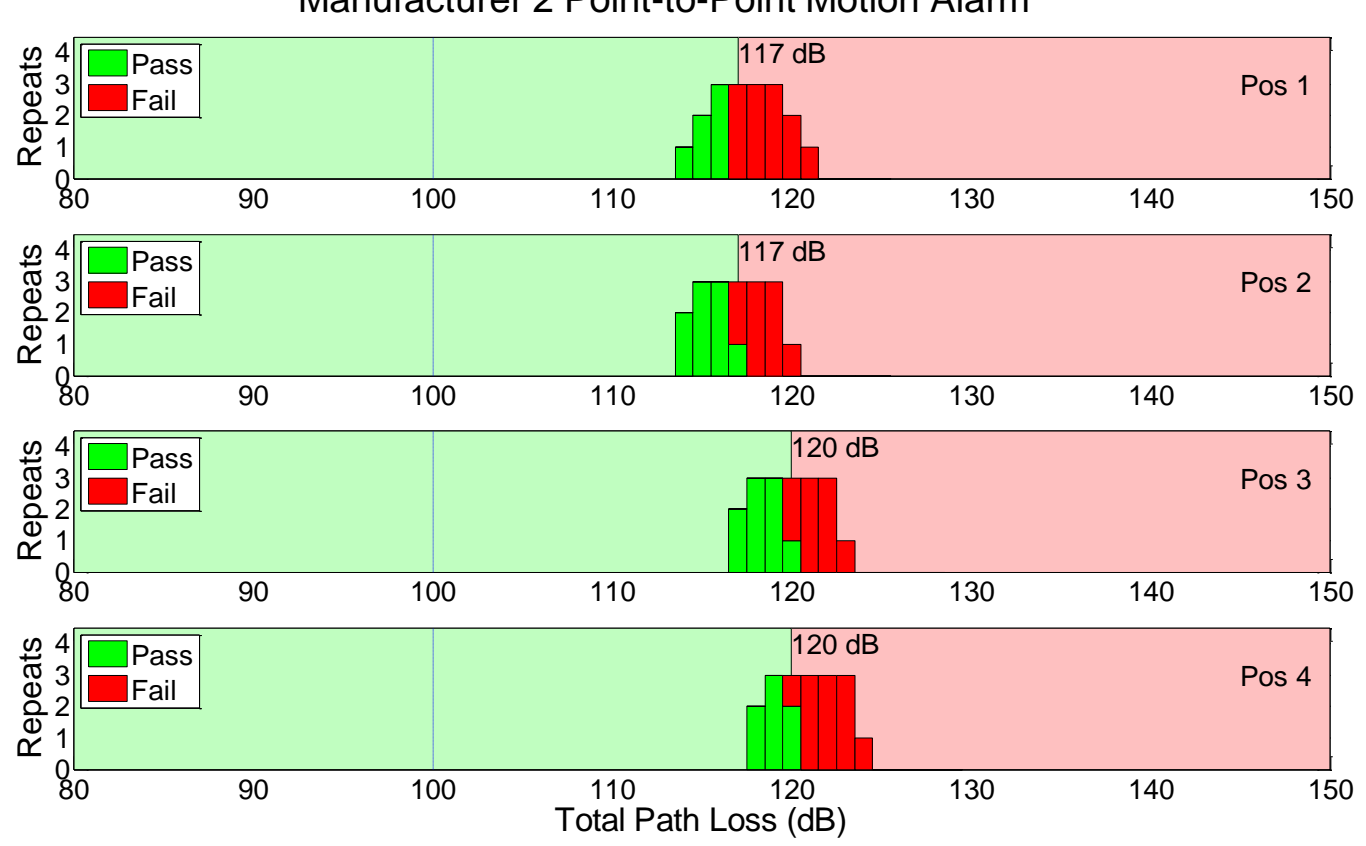

Figure 28. Point-to-Point Motion Alarm test for Manufacturer 2. 
Manufacturer 3 Point-to-Point Motion Alarm
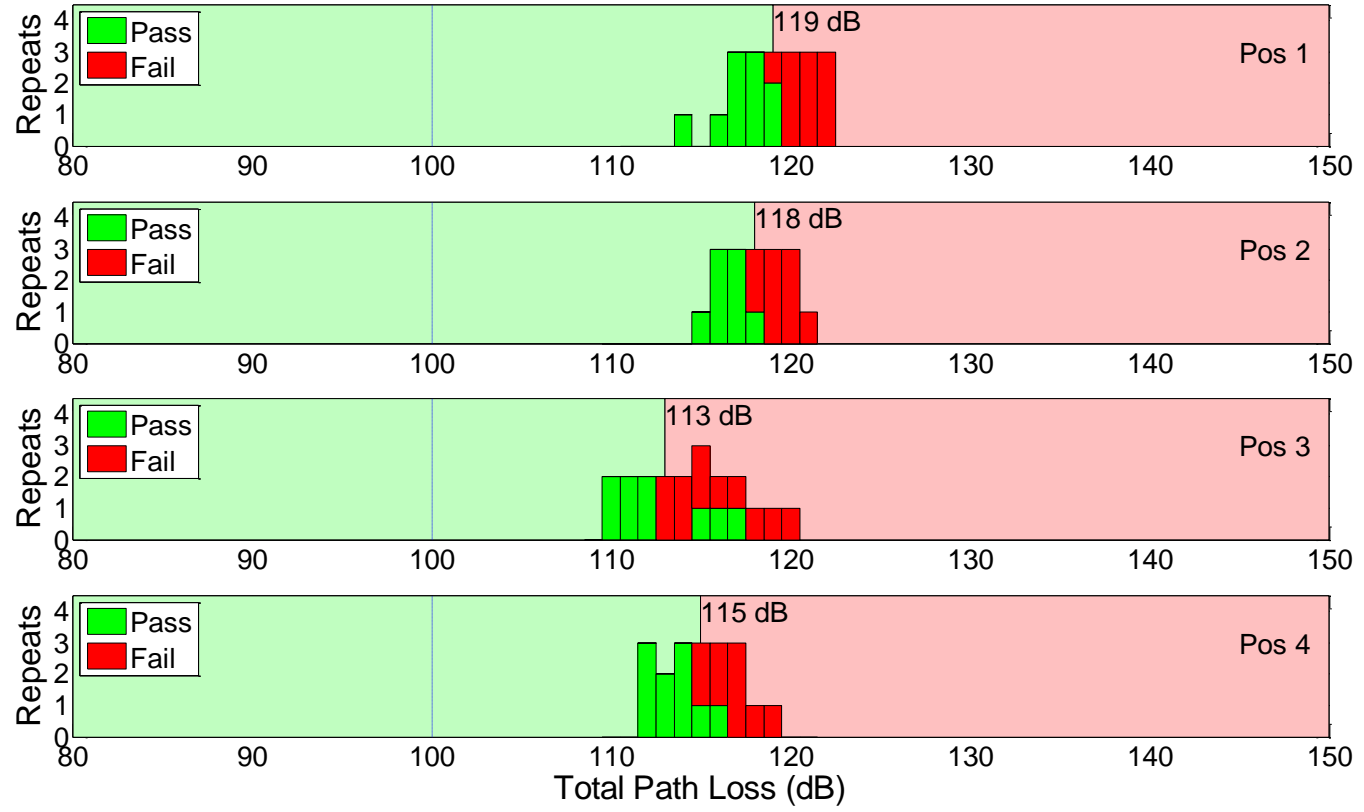

Figure 29. Point-to-Point Motion Alarm test for Manufacturer 3.

Manufacturer 4 Point-to-Point Motion Alarm
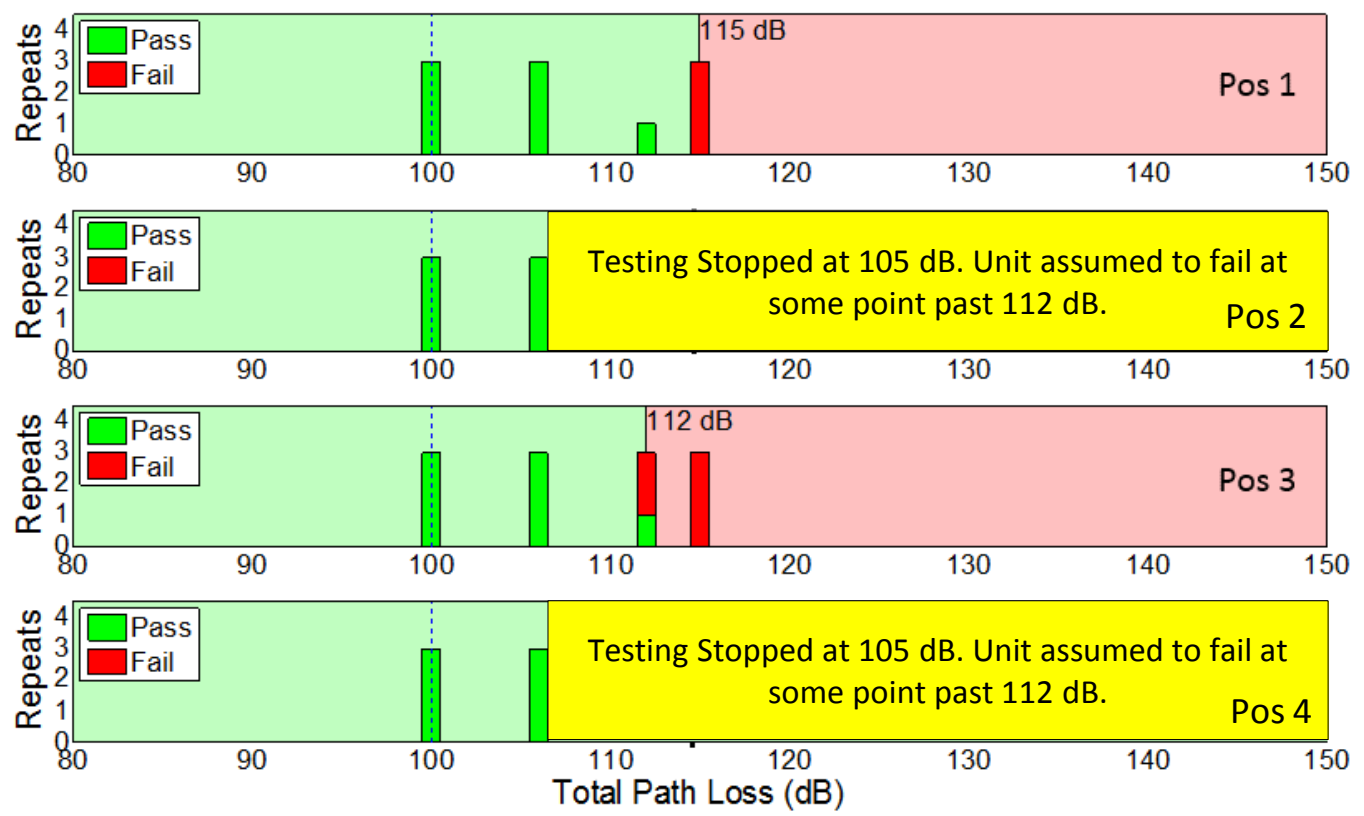

Figure 30. Point-to-Point Motion Alarm test for Manufacturer 4.

Note that each subplot within the figures contain results for the four different orientations of the PASS devices. This indicates that the position of the PASS device has an effect on performance. In the Point-to-Point Motion Alarm test, the position makes a difference as to whether the motion alarm is received within 30 seconds with $100 \mathrm{~dB}$ total path loss. The importance of these tests was 
to ensure there was a margin of successful operation past $100 \mathrm{~dB}$. Manufacturer 4 showed successful operation in positions 2 and 4 up to $105 \mathrm{~dB}$, meeting this $100 \mathrm{~dB}$ requirement. Because the requirement had already been met, we did not need to continue any further, and testing stopped after $105 \mathrm{~dB}$ for position 2 and 4 shown in Figure 4.

\subsubsection{Evacuation Alarm Test Results}

For the Evacuation Alarm Test, we needed to maintain intermittent movement of the portable RF PASS unit so that the motion alarm does not engage. One method to do this is to insert a dielectric rod through the chamber wall to be attached to the RF PASS. This enables the operator to shift the device slightly every 30 seconds or less. When in motion alarm mode, the RF PASS device is unable to receive the evacuation alarm signal because of the priority the motion alarm takes.

One method to enable the operator to send the evacuation alarm signal after the chamber door is closed is with simple timed mouse-click software, installed on the computer that is used with the base station. This software may be used for the $900 \mathrm{MHz}$ and $2.4 \mathrm{GHz}$ systems. The $450 \mathrm{MHz}$ system does not require the mouse-click software because there is no computer. For the $450 \mathrm{MHz}$ system, a second operator may stand in the large anechoic chamber that houses the base station and manually send the evacuation alarm signal with the door closed. Figure 31 through Figure 33 show the results of the Point-to-Point Evacuation test for the first three manufacturers.

Manufacturer 1 Point-to-Point Motion Evacuation
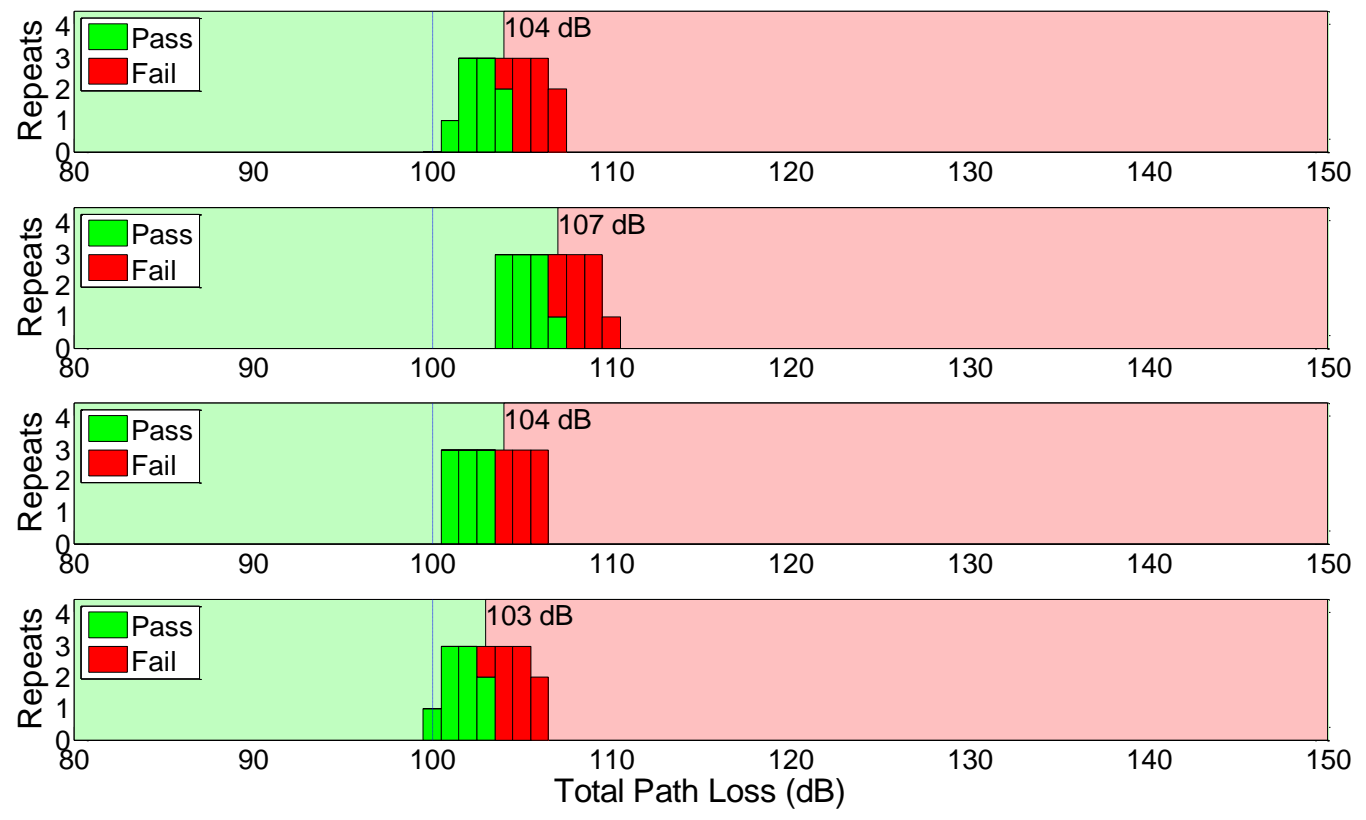

Figure 31. Point-to-Point Evacuation Test for Manufacturer 1. 
Manufacturer 2 Point-to-Point Motion Evacuation
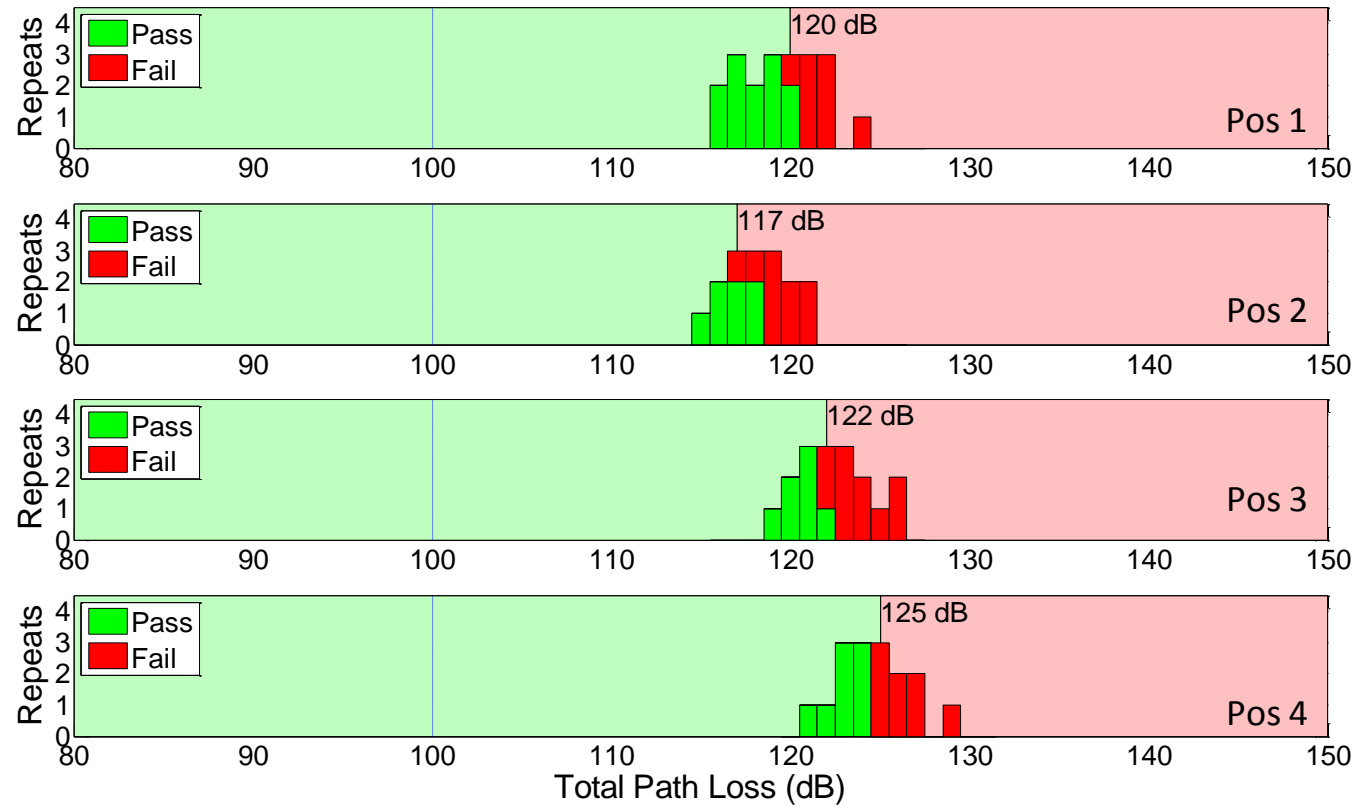

Figure 32. Point-to-Point Evacuation Test for Manufacturer 2.

Manufacturer 3 Point-to-Point Motion Evacuation
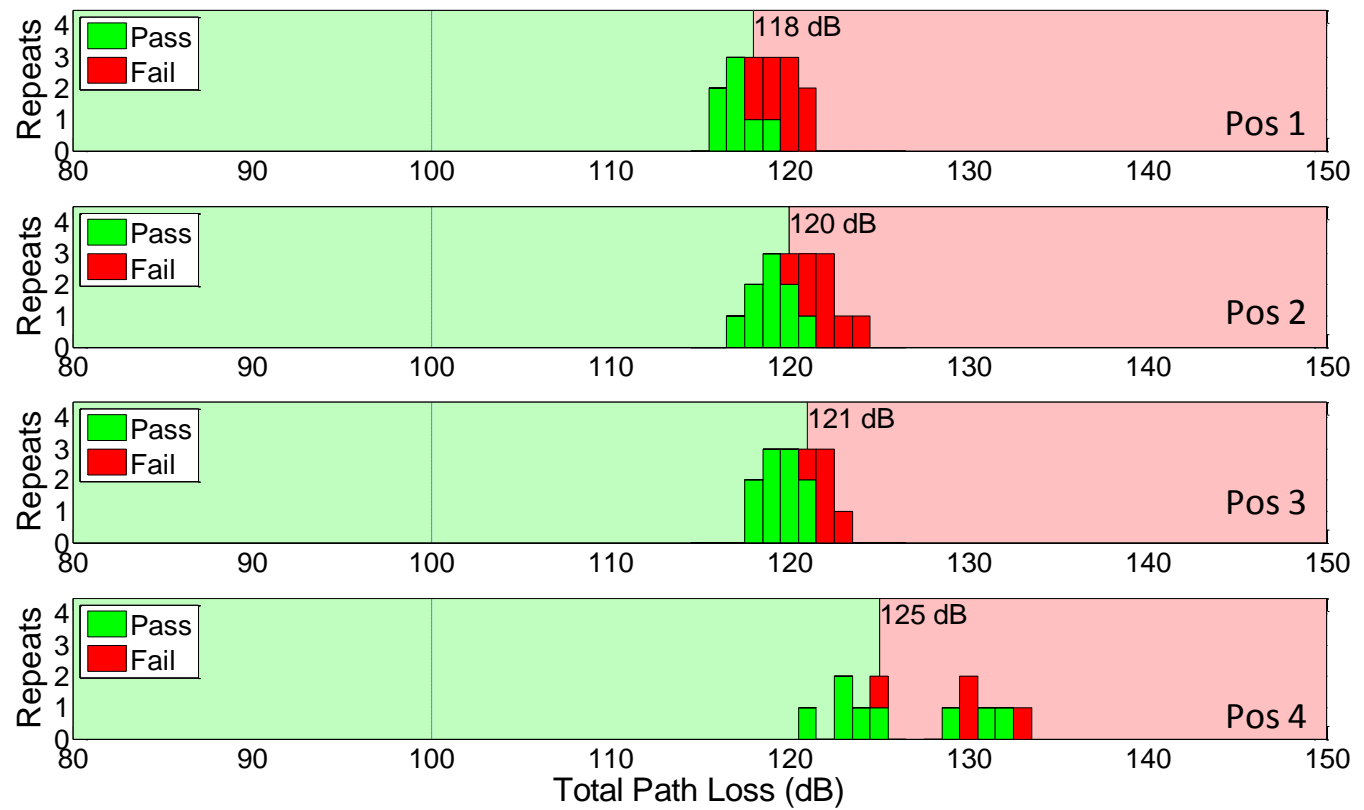

Figure 33. Point-to-Point Evacuation Test for Manufacturer 3.

\subsubsection{Point-to-Point Test Uncertainty Analysis}

This section uses information from the field-uniformity uncertainty results in Section 2.4.3 to create an uncertainty analysis for the Point-to-Point Test. The Point-to-Point Test utilizes the setup 
in Figure 26, which requires consideration of the uncertainty in the field at each of the test platform surfaces and the performance of the inline attenuator. The uncertainty in measurement of the variable attenuator is considered a Type A uncertainty because manufacturer specifications are provided. The overall uniformity uncertainty calculated in Section 2.4.3 used Type A and B methods. As such, the overall uniformity uncertainty is a Type B uncertainty. The following calculations utilize equation (4).

\subsubsection{Combined Uncertainty}

Calculations to find mismatch loss in Table 19 use equation (16):

$$
\operatorname{Mismatch} \operatorname{Loss}(\mathrm{dB})=-10 \times \log _{10}\left(1-\left[\frac{V S W R-1}{V S W R+1}\right]^{2}\right)
$$

Table 19. Description of uncertainties for Point-to-Point Test.

\begin{tabular}{|c|l|l|c|c|c|}
\hline Type & Variable Name & $\begin{array}{c}\text { Uncertainty } \\
\text { Description }\end{array}$ & Method of Estimate & $\begin{array}{l}\text { Values } \\
\text { (Linear } \\
\text { Scale) }\end{array}$ & $\begin{array}{l}\text { Values } \\
(\mathbf{d B})\end{array}$ \\
\hline A & $u_{\text {variable attenuator }}$ & $\begin{array}{c}\text { Uncertainty in } \\
\text { mismatch } \\
\text { losses of } \\
\text { variable } \\
\text { attenuator }\end{array}$ & $\begin{array}{c}\text { Mismatch loss calculated } \\
\text { based on SWR }(1.15) \\
\text { given by manufacturer. }\end{array}$ & $<0.5 \%$ & 0.02 \\
\hline B & $u_{\text {uniformity uncertainty }}$ & $\begin{array}{c}\mu_{\text {combined }} \\
\text { from Section } \\
2.4 .3 .\end{array}$ & $\begin{array}{c}\mu_{\text {combined }} \text { from Section } \\
2.4 .3 .\end{array}$ & 0.33 & 2.48 \\
\hline
\end{tabular}

$$
\sqrt{u_{\text {uniformity uncertainty platform } 1}^{2}+u_{\text {variable attenuator }}^{2}+u_{\text {uniformity uncertainty platform } 2}^{2}} .
$$

This results in an estimated uncertainty of $3.35 \mathrm{~dB}$. This is $0.35 \mathrm{~dB}$ higher than the $3 \mathrm{~dB}$ in the uniformity test which is to be expected since two separate chambers have been connected. Note that although the uniformity uncertainty is considered twice, the overall uncertainty increased by much less than a factor of two (i.e., $3 \mathrm{~dB}$ ). 


\subsection{Multi-Hop Test}

The purpose of the Multi-Hop Test is to verify that an RF PASS device can relay information to another RF PASS device through two repeaters under a certain amount of path loss and multipath. This test is designed so that the signal from RF PASS A must travel through each of the repeaters $\mathrm{B}$ and $\mathrm{C}$, to reach the base station. We performed the Multi-Hop Test using two different RF PASS systems. At NIST, the same RF absorbing lens used in Figure 10(a) was used in the Multi-Hop Test setup, which is shown in Figure 34.

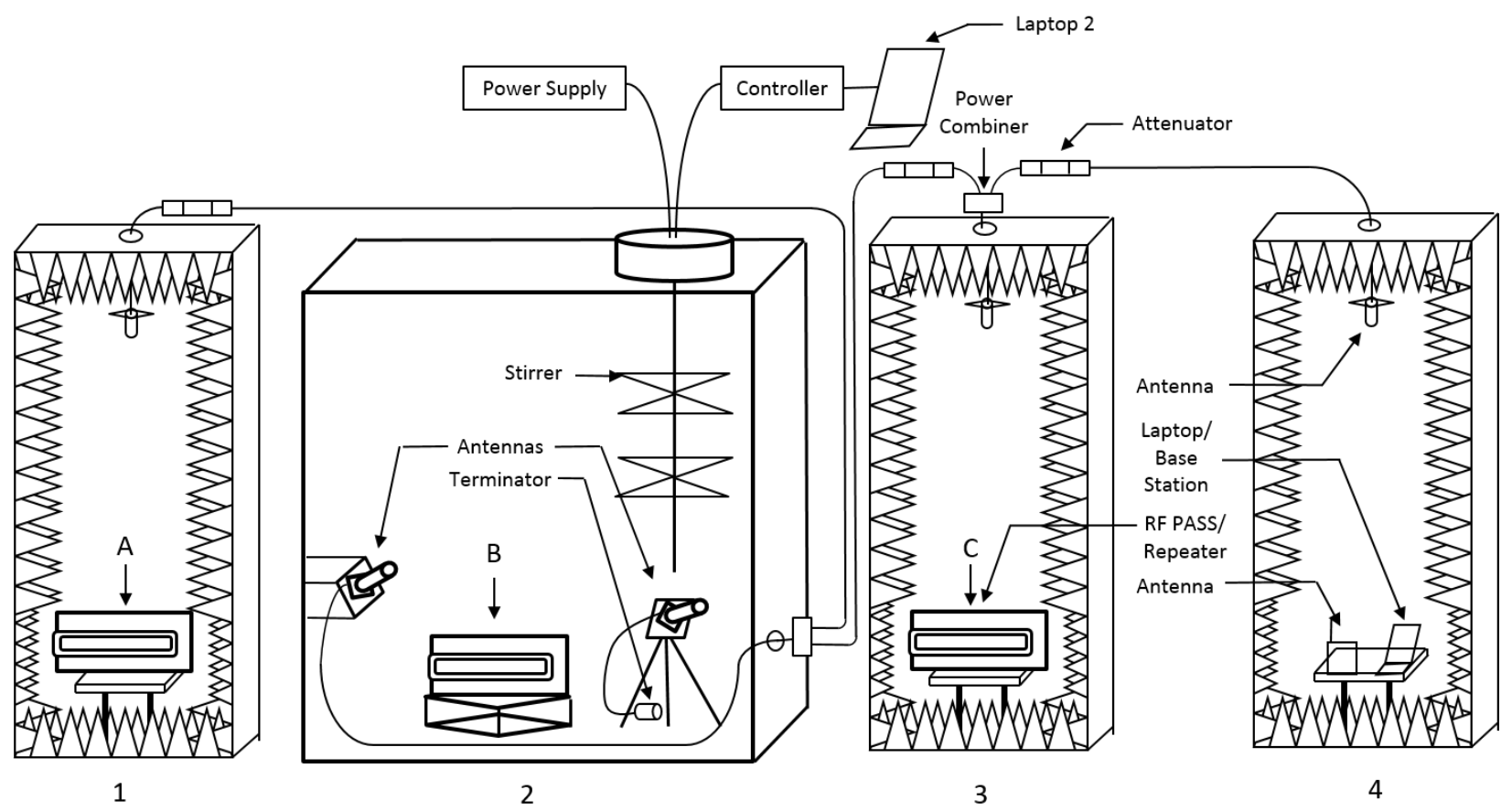

Figure 34. Setup for connection of Multi-Hop Test with four chambers.

\subsubsection{Description}

For Manufacturer 2 and Manufacturer 3, the stirrer in the reverberation chamber was rotating at 3 revolutions per minute in all test cases. To test that the RF isolation between chambers was sufficient to force signal relay through the repeaters, the cables between chambers were disconnected and reconnected. The RF PASS in chamber 1 and repeater in chamber 3 were each placed horizontally or vertically to create four different position combinations. The repeater in chamber 2 was consistently horizontal. When disconnected, the RF PASS communication link to the base station failed, and when reconnected, it was reestablished. The chamber containing device $\mathrm{B}$ was then disconnected and we verified that B and A both were unable to connect, then when the cables were reattached, the connection was successful. Lastly, the chamber containing device C was disconnected from the system and all three devices failed, then reconnected when the cables were reattached. These results prove that the isolation between chambers will force the signals through the designated cable paths.

After verifying isolation, the multi-hop test began. The motion alarm was permitted to activate and the time it took for the base station to receive the motion alarm signal was recorded. If the base 
station received the RF PASS motion alarm within 30 seconds of the alarm being sent, the system was considered to pass. For the evacuation alarm test the evacuation alarm was timed to go off by the automated click software 1.5 minutes after the chamber door was shut. A dielectric rod was used by the operator to prevent the motion alarm from going off. Then, once the evacuation alarm was sent, the time until the RF PASS received the alarm was recorded. If the evacuation alarm was received by the RF PASS within 30 seconds of the alarm being sent from the base station, the system was considered to pass.

For all frequencies, there was a minimum $85 \mathrm{~dB} P L_{t o t}$ between the reverberation chamber and anechoic chamber. Between chambers 3 and 4 there was $100 \mathrm{~dB} P L_{t o t}$. The $85 \mathrm{~dB}$ is the highest attenuation for which the devices could maintain contact with the repeaters. The added attenuation between chambers 1 and 2, and between 2 and 3, was increased in $1 \mathrm{~dB}$ increments to gather the point-of-failure data shown in Figure 35 and Figure 36. Based on these results, we determined that only $80 \mathrm{~dB}$ of attenuation was required between the reverberation chamber and anechoic chamber for an overall $P L_{t o t}$ of $260 \mathrm{~dB}$. As shown in the graphs below, there are never any failures for a total path loss of $260 \mathrm{~dB}$.

\subsubsection{NIST Results}

All results are shown in Figure 35 and Figure 36.

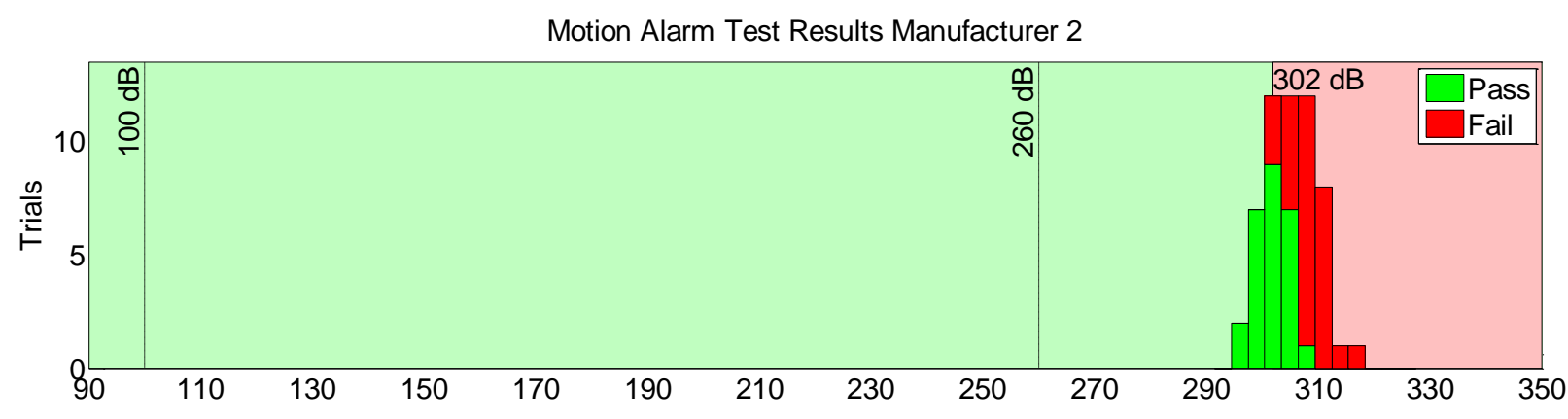

Motion Alarm Test Results Manufacturer 3

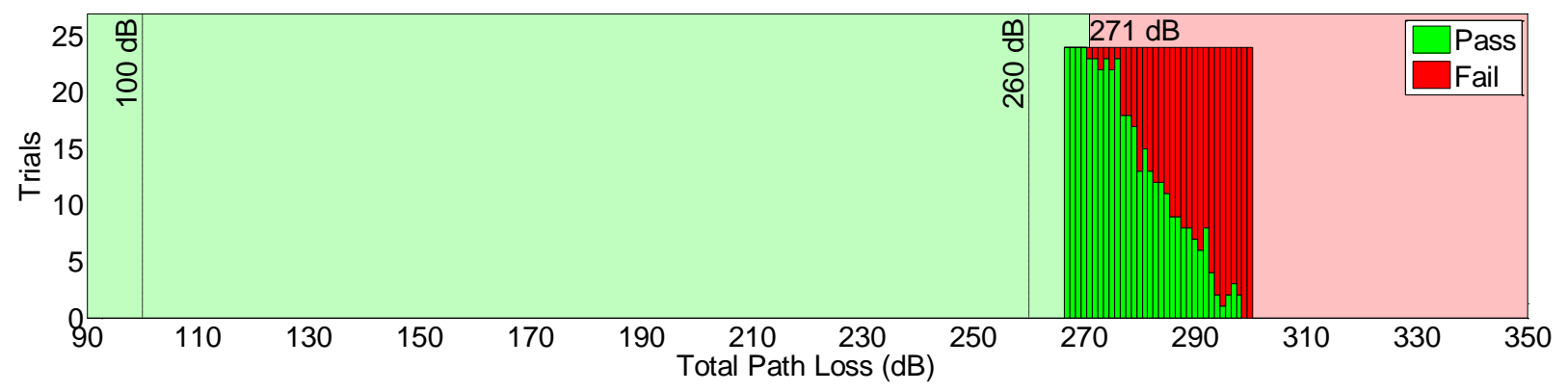

Figure 35. Aggregate point-of-failure results for the motion alarm test for Manufacturers 2 and 3. Dashed lines represent the total path loss required by [1] for the Point-to-Point and Multi-hop Tests, respectively. 
Evacuation Alarm Test Results Manufacturer 2

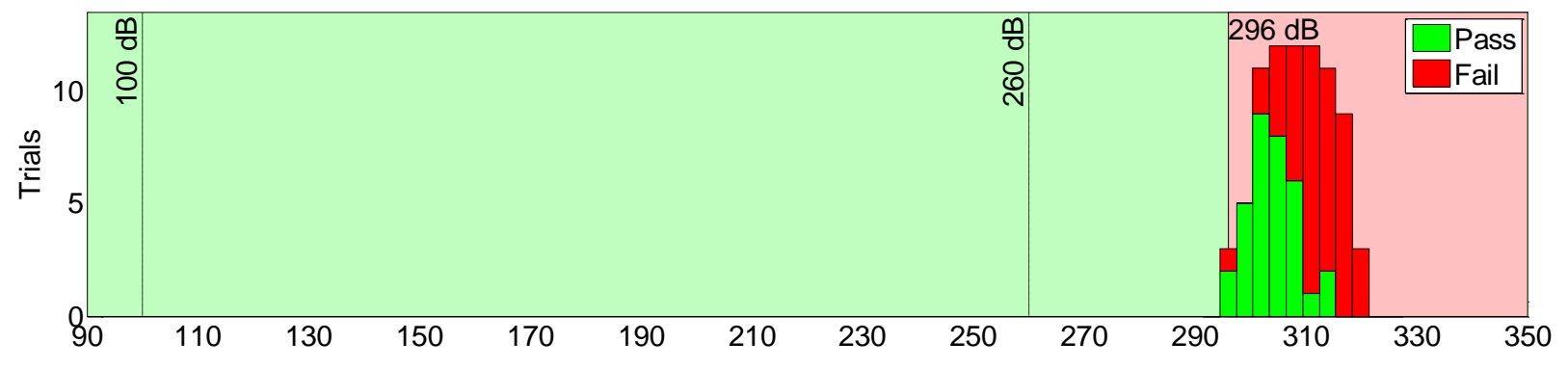

Evacuation Alarm Test Results Manufacturer 3

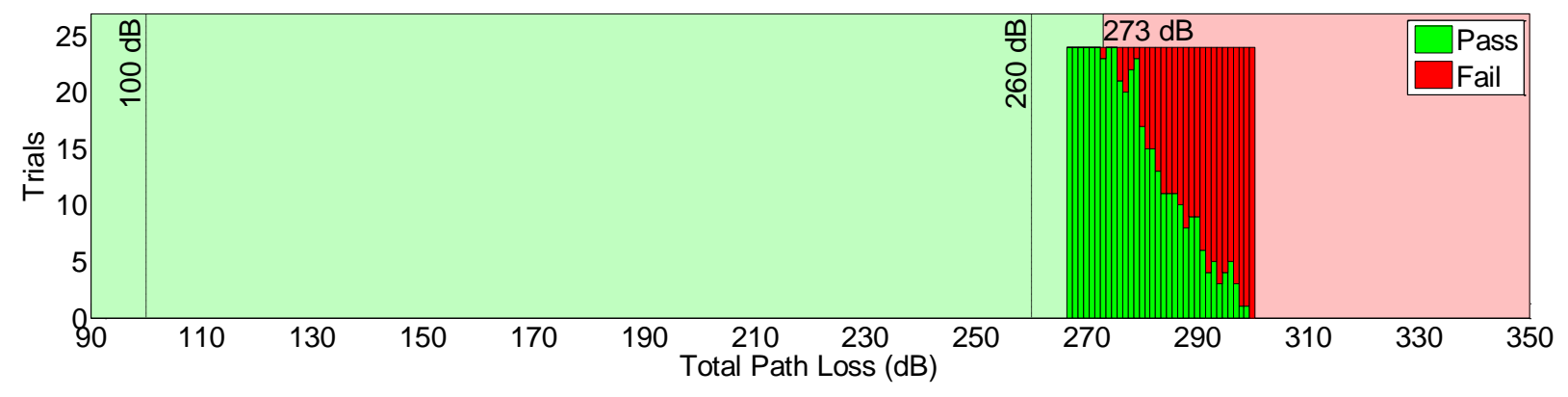

Figure 36. Aggregate point-of-failure results for the evacuation alarm test of Manufacturers 2 and 3. Dashed lines represent total path loss required for Point-to-Point and Multi-hop tests, respectively.

\subsection{Low-Power Interference Testing}

\subsubsection{Introduction}

The RF Low-Power Interference Test is designed to introduce into the RF propagation channel the types of interference that may be found in environments where firefighters are deployed. This test, which was previously discussed in [3], focuses on replicating conditions for large building structures such as office buildings, factories, convention centers, and apartment buildings. Certain wireless transmissions that may cause interference are commonly found within these structures. For example, in offices and apartment buildings, the use of wireless local-area networks (WLAN) or wireless personal-area networks (WPAN) is common. In warehouses and factories, the use of RFID technology is common.

Wireless systems such as WPAN and RFID operate in the unlicensed Industrial, Scientific, and Medical (ISM) frequency bands, with frequencies and power levels specified by the FCC. Because many RF PASS units also operate within these unlicensed frequency bands, in-band interference is possible. Consequently, the RF Interference Test is designed to test systems that operate in similar frequency bands by use of commonly encountered transmission protocols.

The interfering source in this test method will operate at approximately the same output power as the RF PASS; that is, at the maximum power allowed by the FCC. Higher-power signals that are transmitted either within the same band as the RF PASS (for example, signals that operate in the $900 \mathrm{MHz}$ frequency band that are licensed for land-mobile radio operations) or at frequencies 
other than the RF PASS system (for example, broadcast radio or cellular telephone operations) are not considered in this test method.

As shown in Figure 37, the interfering signal is introduced into the test chamber that contains the user-worn RF PASS. This configuration is tested to simulate the condition where a firefighter is indoors in the presence of some other radio system. Because we expect that the firefighter will typically be some distance from the RF interfering source in this test method, the output power of the interferer is reduced by the free-space path loss corresponding to a $1.25 \mathrm{~m}$ distance. This distance was chosen as the closest expected proximity between a firefighter and another wireless device. Note that this distance falls within the range of distances proposed in similar work on medical device RF interference testing discussed in [19] [20].

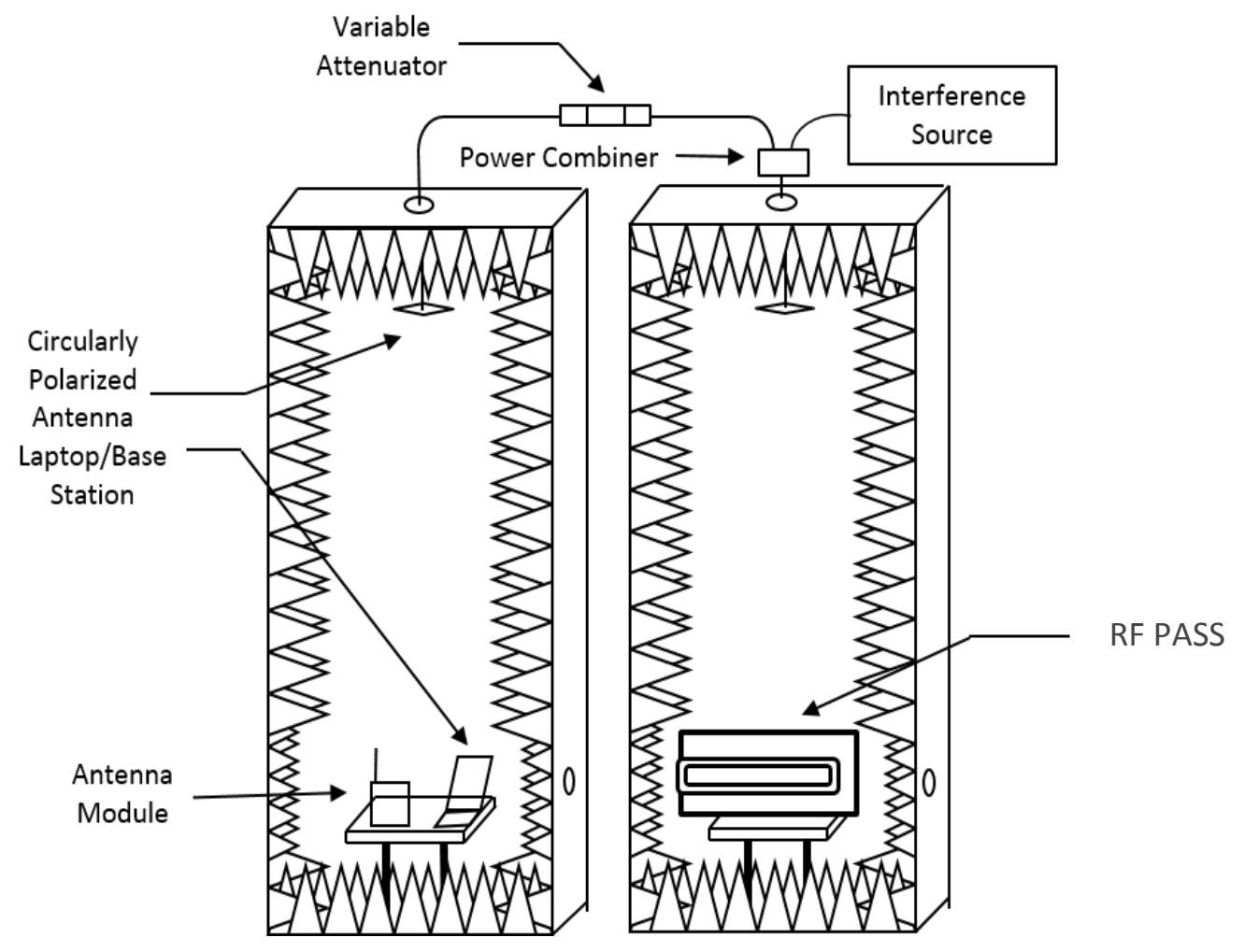

Figure 37. A typical RF Low-Power Interference Test set up for RF PASS. The RF interference source is connected via a power combiner to the antenna located at the top of the chamber containing the RF PASS portable unit.

As with the Point-to-Point Attenuation Test, this test method is designed to allow free-field testing of a complete RF PASS system without the use of conducted measurements or removal of the antennas. Free-field testing allows the system to be characterized with any unusual antenna radiation pattern intact.

Finally, we point out that interference testing has been reported in prior literature: for the $900 \mathrm{MHz}$ ISM band, see [19][21] and for the $2.4 \mathrm{GHz}$ ISM band, see [20][22][23]. In addition, [23] performed laboratory based coexistence testing in the $2.4 \mathrm{GHz}$ ISM band for medical applications. All of the aforementioned work utilized several elements similar to those of the test method we 
describe here, such as the use of an anechoic chamber to control the test environment and the use of commercial wireless devices as representative interference sources. In the future, it may be possible to merge some of the testing concepts, such as the channel occupancy (discussed here) and the transaction "breakdown" (discussed in [23]).

\subsubsection{The Target Value of Interference}

The interference tests described below focus on two primary frequency bands and transmission formats. These target values of interference are detailed in Table 20. The transmission formats used in this test (including power level, modulation, encoding schemes, and signal bandwidth) have been designed to replicate those of commonly-found wireless devices. As designed, the interference source is active 50 percent of the time in either frequency band (e.g., over the $902 \mathrm{MHz}-928 \mathrm{MHz}$ band), or the initial channel of operation (e.g., over one of the six IEEE $802.11 \mathrm{~b} / \mathrm{g} 20 \mathrm{MHz}$ channels, numbered 1, 3, 5, 7, 9, 11).

Because the anticipated channel usage by the interferer in an actual deployment will vary from instant to instant, we statistically verify the target value of interference used in testing. We define 50 percent channel usage such that a spectrum analyzer measurement over a 30 -second period will detect the presence of the interference source 50 percent of the time, with the remaining samples measuring a clear or interference-free RF channel. In addition, over any five-second interval, the interference should be active between 25 and 75 percent of the time. Figure 38 shows an example based on the specified criteria for a $2.4 \mathrm{GHz}$ interference source.

Table 20. Interference sources for RF PASS testing in the $900 \mathrm{MHz}$ and $2.4 \mathrm{GHz}$ ISM bands.

\begin{tabular}{|c|c|c|c|}
\hline $\begin{array}{c}\text { Frequency } \\
\text { Range }\end{array}$ & $\begin{array}{c}\text { Transmission Format or } \\
\text { Modulation Scheme }\end{array}$ & $\begin{array}{c}\text { Subcarrier or Channel } \\
\text { Bandwidth }\end{array}$ & Output power and FCC part \\
\hline $\begin{array}{c}902-928 \\
\mathrm{MHz}\end{array}$ & $\begin{array}{c}\text { Frequency Hopping } \\
\text { Spread Spectrum (FHSS) }\end{array}$ & $100 \mathrm{kHz}$ subcarrier & $\begin{array}{c}1 \mathrm{~W} \text { peak power (30 dBm) into } \\
\text { antenna (w/ max 6 dBi gain), FCC } \\
\text { Part 15.247 [24] }\end{array}$ \\
\hline $\begin{array}{c}2.4-2.472 \\
\mathrm{GHz}\end{array}$ & $\begin{array}{c}\text { Direct Sequence } \\
\text { Spread Spectrum } \\
\text { (DSSS) }\end{array}$ & $\begin{array}{c}20 \mathrm{MHz} \text { (IEEE } \\
802.11 \mathrm{channels)}\end{array}$ & $\begin{array}{c}63 \mathrm{~mW} \text { peak power (18 dBm) into } \\
\text { antenna, FCC Part 15 (See example 2 } \\
\text { [24] for determination of correction } \\
\text { factor.) }\end{array}$ \\
\hline
\end{tabular}




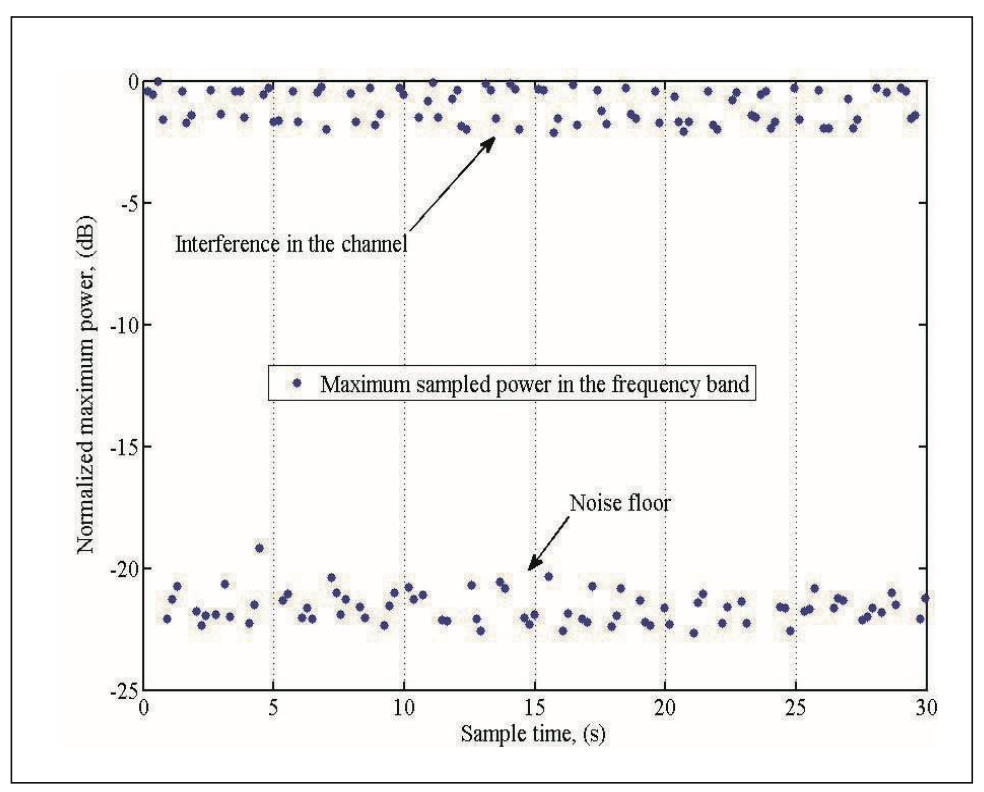

Figure 38. An example measurement showing 50 percent channel usage over a 30-second interval. The sampling rate was approximately $190 \mathrm{~ms}$, and the 5-second intervals delineated by the dashed lines indicate active interference between 40 and 60 percent of the time within the interval. The measurement of a "noise" value means that the channel is clear of interference.

The channel usage percentage is measured with a spectrum analyzer and data acquisition software that samples the spectrum at the rate described above. In our case, the spectrum analyzer sweeps across the frequency band of interest in less than $3 \mathrm{~ms}$; the data acquisition software captures the spectrum with a sampling rate of $225 \mathrm{~ms} \pm 50 \mathrm{~ms}$, and searches for the maximum value within the captured spectrum. Only the interference source is active when determining the interference channel usage; that is, there is no RF PASS communication activity. To arrive at the statistics for the target interference, a minimum of 500 samples are collected over approximately two minutes of data acquisition. The power threshold was $15 \mathrm{~dB}$. The resolution bandwidth should be as low as possible while still achieving a $3 \mathrm{~ms}$ sweep time. In our case, $110 \mathrm{kHz}$ and $180 \mathrm{kHz}$ resolution bandwidth was used for the $900 \mathrm{MHz}$ and $2.4 \mathrm{GHz}$ frequencies, respectively. The ratio of interference signal samples to the noise samples provides the channel usage percentage. As discussed above, the channel usage percentage may vary in any five-second interval between 25 and 75 percent. The test configuration for the RF interference source based on the use of standard commercial wireless products is included in the measurement description that follows.

\subsubsection{Measurement System}

Figure 39 shows a typical RF Interference Test set up. Two anechoic chambers provide shielding between the portable unit and the base station. The total path loss (or gain) associated with the environmental elements simulates the path loss experienced by personnel carrying RF PASS within a building or structure when the base station is located outside. The value of the external attenuator is adjusted in a calibration step described in the section entitled "System Calibration: Target Path Loss." For the example results shown below, a $100 \mathrm{~dB}$ total path loss was inserted between the base station and portable RF PASS. Note that the attenuation path now includes the 
power combiner, and so the external attenuator value must be changed from that used in the Pointto-Point Attenuation Test.

The interferer is connected to the test chamber containing the user-worn device through a coaxial cable connected to the power combiner. The loss due to the coaxial cable and power combiner must be added to the nominal output power specified in Table 20, above.

\subsubsection{Specific Interference Test Configurations for $900 \mathrm{MHz}$ and 2.4 GHz Systems}

This section provides specifics on setting up the interference sources used in testing the RF PASS devices. Note that in both the $900 \mathrm{MHz}$ frequency-hopping, spread-spectrum (FHSS) and $2.4 \mathrm{GHz}$ direct-sequence, spread-spectrum (DSSS) interference tests, the RF data rates are intentionally low in order to create high usage of the RF wireless channel by the interfering device. Most wireless systems are designed to maximize data throughput while minimizing the usage of the wireless channel to the greatest extent possible. This optimization is achieved, in part, by choosing a modulation format that allows the system to transmit the most data for the detected signal-tonoise ratio. The lower the signal-to-noise ratio, the lower the data throughput. If a lowerthroughput modulation format is chosen, the transmission will require more time, and thus occupy the channel longer while transmitting the same amount of data. Here, we are intentionally inefficient in our usage of the RF wireless channel in order to mimic high-usage conditions. The amount of wireless-channel activity in terms of RF transmission power levels and duration is important here, not the amount of data transferred over the wireless link.

Table 21 provides specifics for the $900 \mathrm{MHz}$ frequency-hopping interference test. The interference source is a wireless development board that utilizes industrial wireless transceivers, and is intended to represent a typical interference source that may be encountered during the deployment of an RF PASS system. As shown in Table 21, the key parameter for varying the interference duty cycle is the hop duration, or time that the interfering signal lingers on a particular frequency. A $19 \mathrm{~ms}$ hop duration creates the 50 percent channel usage with the statistical behavior described above. The $900 \mathrm{MHz}$ interference source used here is a DNT900 series wireless development board from RF Monolithics, Inc. previously employed as an RF interference source in [19]. ${ }^{2}$

Table 21. Parameters for the $900 \mathrm{MHz}$ interference source.

\begin{tabular}{|c|c|c|}
\hline Fixed Parameters & Hop Duration (ms) & Interferer Channel Usage (percent of time) \\
\hline \multirow{3}{*}{$\mathrm{RF}$ channel bandwidth $=100$} & 16 & 57 \\
\cline { 2 - 3 } $\mathrm{kHz}$ & 18 & 53 \\
\cline { 2 - 3 } $\mathrm{RF}$ data rate $=38.4 \mathrm{~kb} / \mathrm{s}$ & 19 & 50 \\
\cline { 2 - 3 } Serial data rate $=38.4 \mathrm{~kb} / \mathrm{s}$ & 20 & 47 \\
\cline { 2 - 3 } Power level $=30 \mathrm{dBm}$ & 22 & 45 \\
\cline { 2 - 3 } & 24 & 39 \\
\hline
\end{tabular}

2 Disclaimer: Mention of any company names serves only for identification, and does not constitute or imply endorsement of such a company or of its products by NIST. Other products may work as well or better. 
The 2.4 GHz DSSS interference set up differs slightly from the set up shown in Figure 37. In this case, the interference source is established by connecting two wireless access points and then passing data between the two devices. The combined output power constitutes the RF interference source, which is connected to the chamber containing the portable RF PASS device in the same manner as in the previous configuration. Figure 39 shows the interference test set up that utilizes two access points.

In these tests, the access points were devices that can operate in multiple IEEE 802.11 configurations. The devices are set up in a bridging mode to allow "ping" packets between the two access points. Use of two access points in bridging mode and at equal distances from the RF PASS allows the maximum testing range (up to near 100 percent channel usage), and thus supports testing of the RF PASS to failure, if so desired. This also allows testing for lower channel-usage values, such as the proposed 50 percent, which simulates the channel usage of multiple wireless devices connected to a single wireless access point on the same channel.

The devices are given unique IP addresses on the same subnet, and the security filters are set to allow the connection between the two devices. The computer is connected via an Ethernet port to one of the access points, which then repeatedly "pings" the other access point with the "continuous ping" option set. The ping packet size is adjusted to achieve the desired channel usage with the packet size option in the ping protocol. Table 22 lists the parameter settings for various interference channel usage values. A ping packet size of $28 \mathrm{~kb} / \mathrm{s}$ corresponds to the 50 percent channel usage described above. The results provided here are based on D-Link® DAP2553 access points [25].

\subsubsection{Measurement Procedure for Interference Testing}

The previous section provided details on how to configure commercial wireless devices to create the desired RF interference behavior. Testing of the RF PASS system is carried out in almost the same manner as the Point-to-Point Attenuation Tests, but with the addition of the appropriate interference source. 


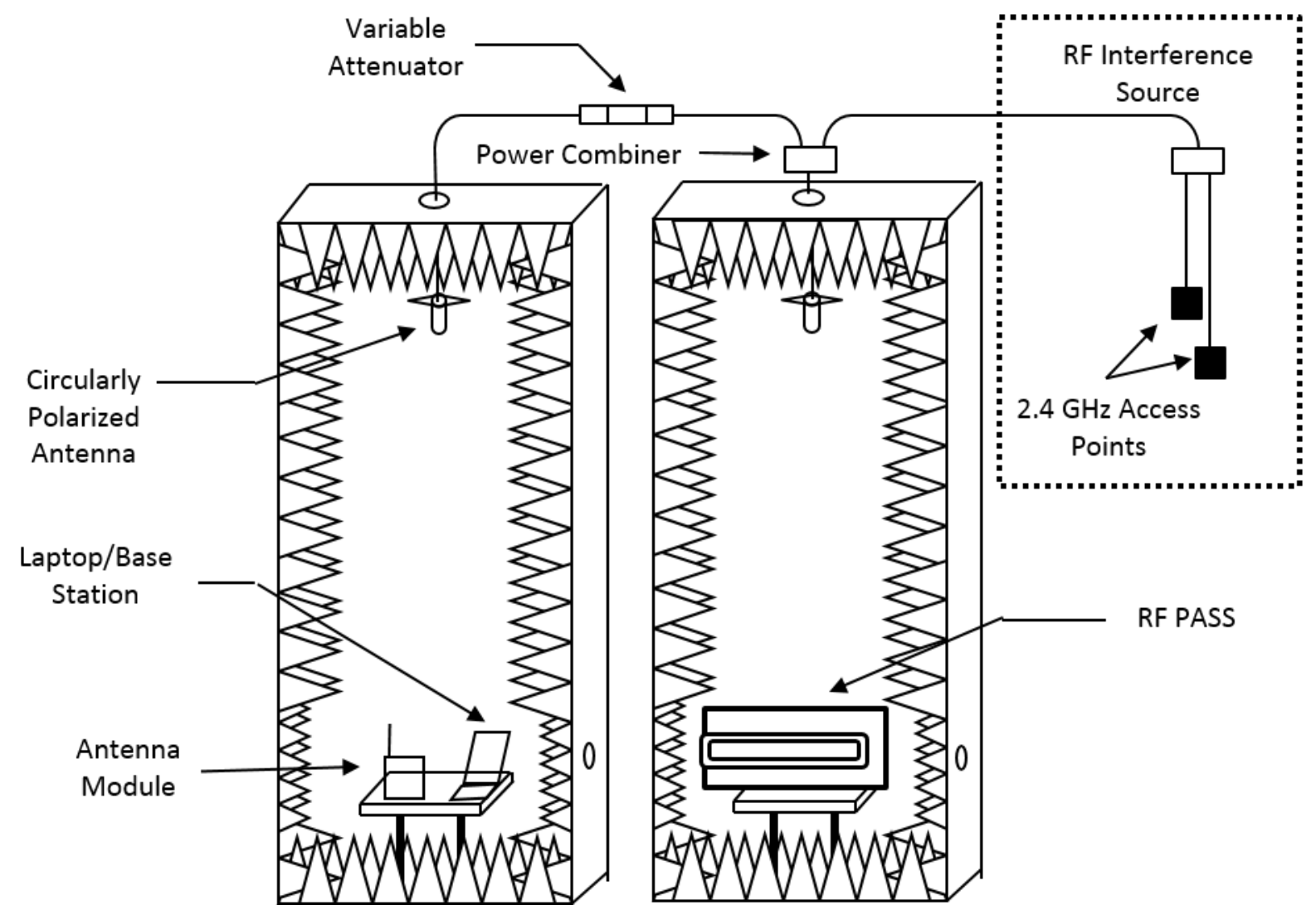

Figure 39. RF interference testing set up for the $2.4 \mathrm{GHz}$ frequency band. Two access points are connected together through a power combiner. The combined signal is then connected to the power combiner that feeds the chamber containing the portable RF PASS unit.

Table 22. Parameters for the $2.4 \mathrm{GHz}$ interference tests with two access points.

\begin{tabular}{|c|c|c|}
\hline Access Point Settings & $\begin{array}{c}\text { Ping Packet Size } \\
(\mathrm{kb} / \mathrm{s})\end{array}$ & Interferer Channel Usage (percent of time) \\
\hline \multirow{6}{*}{$\begin{array}{c}\text { Mode }=\text { Wireless Distribution } \\
\text { System/Bridging }(\text { WDS }) \\
\text { Physical layer }=\text { IEEE } \\
802.11 \mathrm{~b} / \mathrm{g} \\
\text { RF Channel }=1 \mathrm{Mb} / \mathrm{s} \\
\text { Power level }=18 \mathrm{dBm}\end{array}$} & 20 & 35 \\
\hline & 22 & 38 \\
\hline & 24 & 42 \\
\hline & 26 & 45 \\
\hline & 28 & 50 \\
\hline & 30 & 53 \\
\hline
\end{tabular}

The test is conducted for any of the four Attenuation Test positions of the portable RF PASS device under the assumption that the system has successfully passed the Attenuation Test. The base station is again positioned with its antenna lying horizontally on the table. This orientation is designed to maximize the signal level received by the antenna at the top of the chamber.

The test method is conducted as follows: A wireless link is established between the base station and portable RF PASS device. The doors are then closed and the interfering source is turned on. The test administrator simply waits 30 seconds until the motion alarm automatically triggers. The 
test is passed if the base station receives the alarm within 30 seconds, during which the interference source is active, as determined by an audible alarm emitted from the base station.

\subsubsection{NIST Results}

Interference testing was performed on products from three different RF PASS manufacturers. One system operated in the $2.4 \mathrm{GHz}$ ISM band with a DSSS modulation approach; the other two systems used FHSS modulation in the $900 \mathrm{MHz}$ ISM band. The 50 percent channel usage of each interference source allows a basic comparison of the systems.

In Figure 40, the first two RF PASS manufacturers use FHSS in the $900 \mathrm{MHz}$ band. The top graph indicates that the first manufacturer consistently fails to successfully transmit the PASS motion alarm when the interference source is active more than 40 percent of the time. However, as shown in the middle graph, the second manufacturer successfully transmits the motion alarm with active interference present 80 percent of the time. As shown in the bottom graph, the third manufacturer, who uses DSSS in the $2.4 \mathrm{GHz}$ band, successfully transmits PASS motion alarms with interference channel usage up to approximately 60 percent. This system experiences intermittent failures with between 65 and 80 percent channel usage, and it experiences complete failure when the channel usage is 90 percent or more of the time.
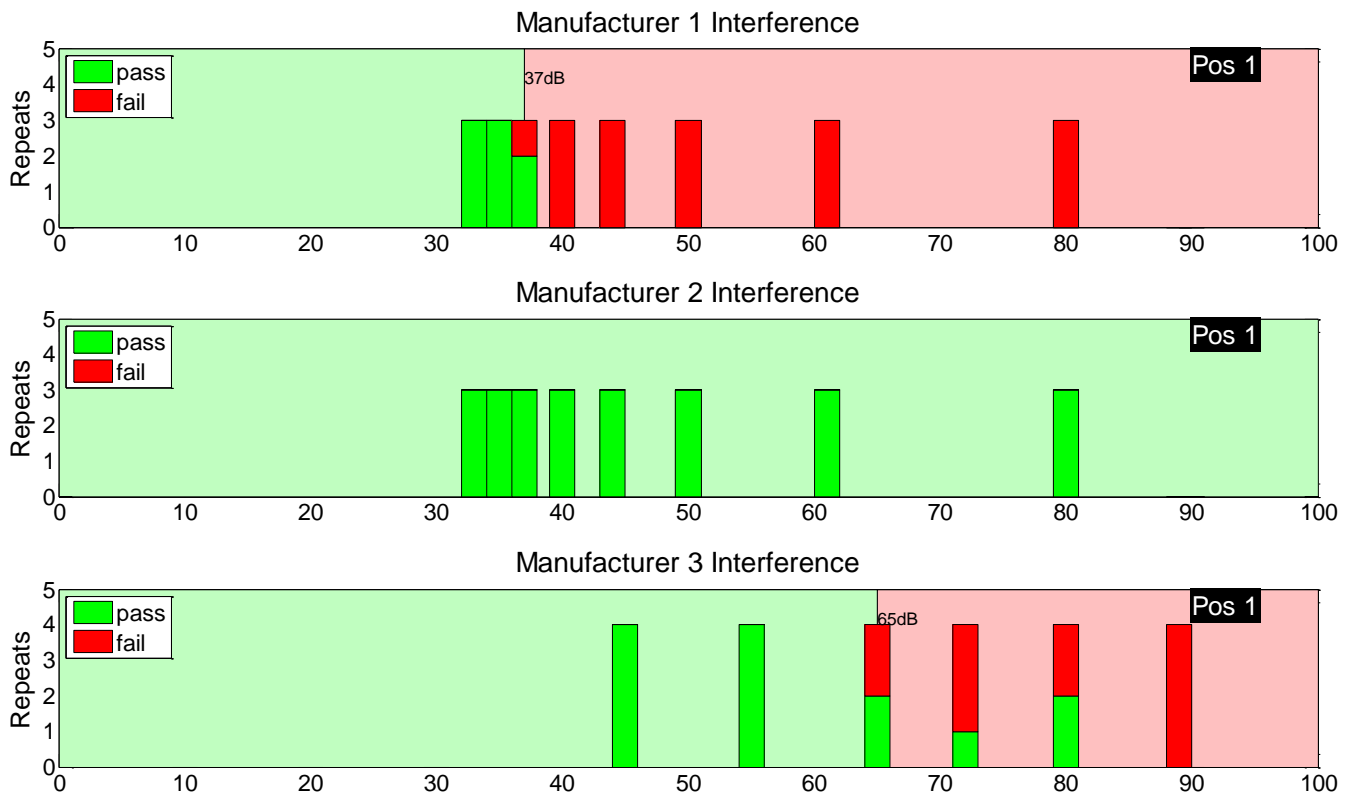

Figure 40. RF Interference Test results based on the channel usage (occupancy) criteria. RF PASS manufacturers 1 and 2 both operate in the $900 \mathrm{MHz}$ band with a frequency-hopping, spread-spectrum modulation format; RF PASS manufacturer 3 operates in the $2.4 \mathrm{GHz}$ band with a direct-sequence, spreadspectrum modulation format.

The test results clearly indicate that (1) successfully transmitting RF PASS motion alarms under the specified interference conditions is possible; (2) the RF Interference Test provides a quantifiable measure of performance for systems that use different modulation schemes and 
frequency bands; and (3) the test can determine whether manufacturers may need to change their designs for more effective alarm communication in the presence of RF interference.

\subsection{High-Power Interference Testing}

This test uses a high-power wideband signal to interfere with an RF PASS device that is attempting to send a motion alarm to the RF PASS base station. In this test, we verify that there will be no lasting damage done to the RF PASS devices after the high-power interference has been turned off, and that when the interference is turned off the motion alarm will reach the base station.

\subsubsection{Creating the Interfering Signal}

The interfering signal selected for this task is a flat-spectrum broadband signal that covers 200 MHz. The broadband nature of this signal ensures that the entire ISM band for all four frequencies used by the manufacturers of RF PASS are covered. Two signal generators within a single PXI chassis were used to create two $100 \mathrm{MHz}$ broadband signals. The center frequency of each signal was placed to slightly overlap the other so as to resemble one $200 \mathrm{MHz}$ broadband signal.

\subsubsection{Setup, Procedure, and Test}

The High-Power Interference Test uses two broadband signals whose center frequencies are chosen such that there is a slight overlap to achieve a $200 \mathrm{MHz}$ span of interference. This span covers the entire U.S. ISM band for each frequency we are working with. The ISM bands and centers are listed in Table 23.

Table 23. ISM bands and centers.

\begin{tabular}{|c|c|c|}
\hline ISM Band Start & ISM Band End & ISM Band Center \\
\hline $433.05 \mathrm{MHz}$ & $434.79 \mathrm{MHz}$ & $433.925 \mathrm{MHz}$ \\
\hline $902 \mathrm{MHz}$ & $928 \mathrm{MHz}$ & $915 \mathrm{MHz}$ \\
\hline $2400 \mathrm{MHz}$ & $2483.5 \mathrm{MHz}$ & $2441.25 \mathrm{MHz}$ \\
\hline $5725 \mathrm{MHz}$ & $5875 \mathrm{MHz}$ & $5800 \mathrm{MHz}$ \\
\hline
\end{tabular}

The center frequencies of each of the two broadband signals that are used to create the $200 \mathrm{MHz}$ broadband signal are given in Table 24.

Table 24. High-Power Interference Test: Center frequency of each broadband signal.

\begin{tabular}{|c|c|}
\hline Signal 1 Center Frequency & Signal 2 Center Frequency \\
\hline $382.425 \mathrm{MHz}$ & $485.425 \mathrm{MHz}$ \\
\hline $863.5 \mathrm{MHz}$ & $966.5 \mathrm{MHz}$ \\
\hline $2389.75 \mathrm{MHz}$ & $2492.75 \mathrm{MHz}$ \\
\hline $5748.5 \mathrm{MHz}$ & $5851.5 \mathrm{MHz}$ \\
\hline
\end{tabular}

Spectrograms, a specific type of spectrum analyzer plot which shows power levels in relation to function of time and frequency, were acquired at the overall center for each band to show that at the center where the bands meet there is consistent interference with no time or frequency gaps. The SA settings can be seen in Table 25 and the plots can be seen in Figure 41 through Figure 42. 

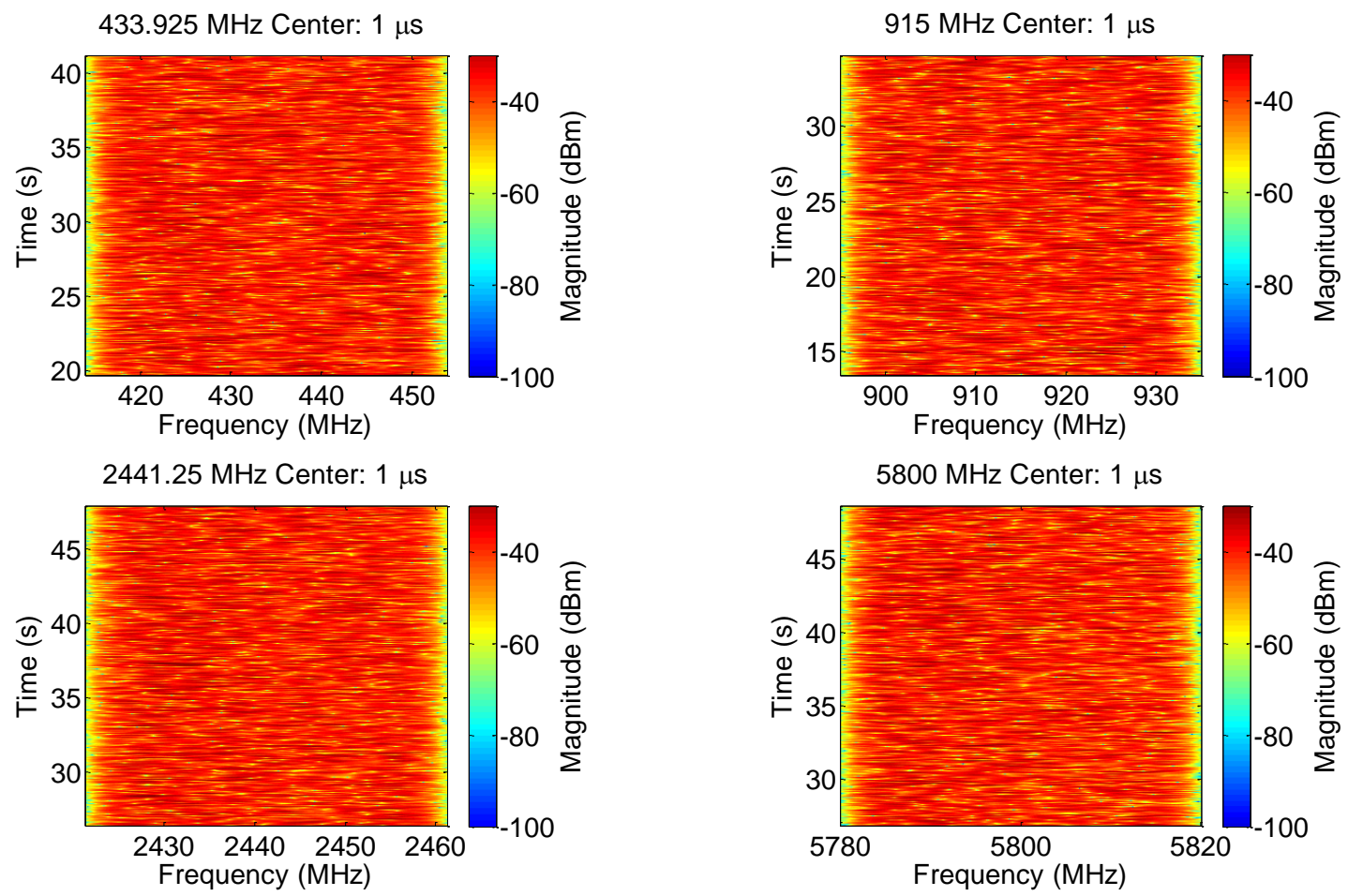

Figure 41. Spectrogram plots at the center of the four frequency bands with $1 \mu$ s time resolution.
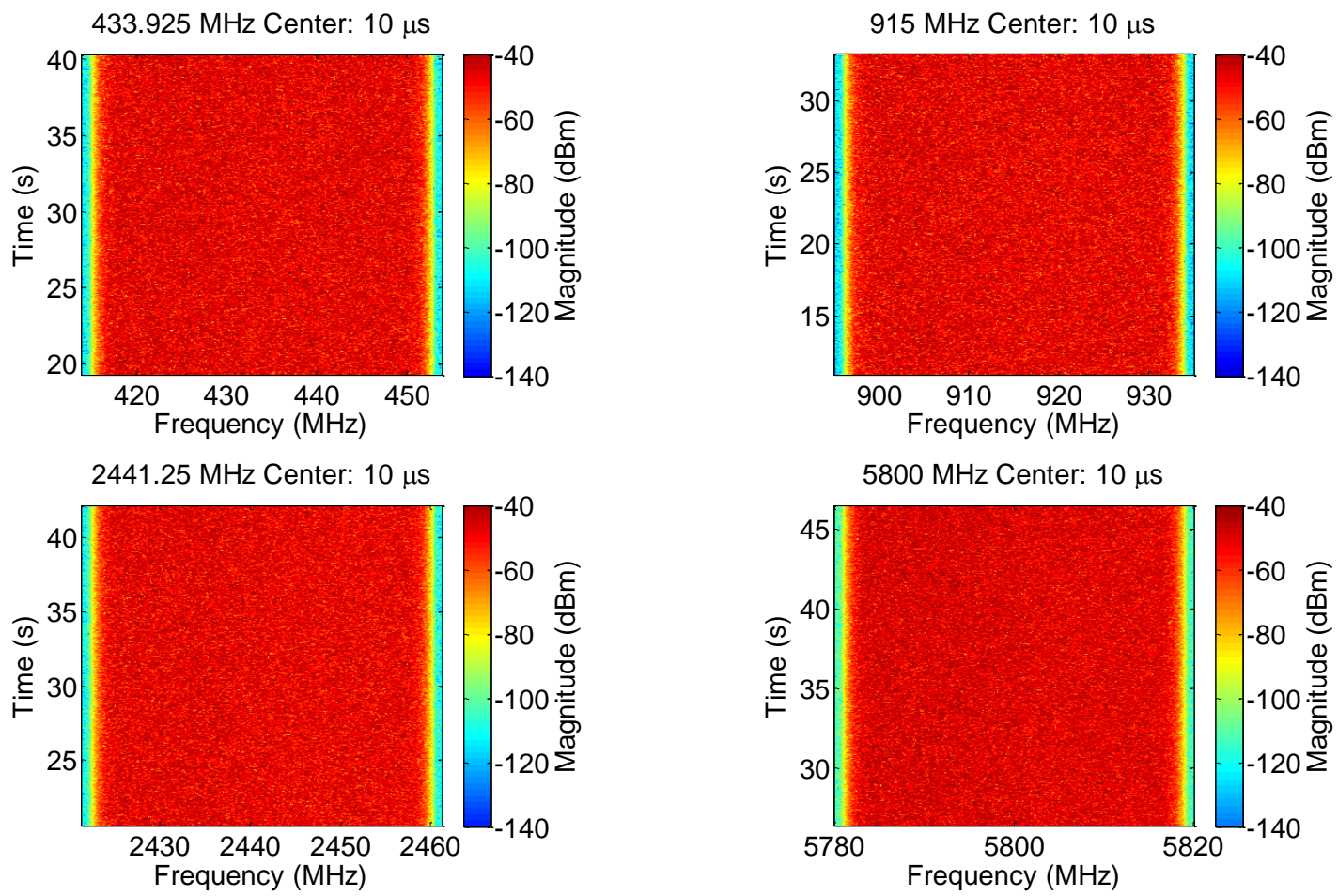

Figure 42. Spectrogram plots at the center of the four frequency bands with $10 \mu$ s time resolution. 
Table 25. Spectrogram settings for the two different captures shown in Figure $41(1 \mu \mathrm{s})$ and Figure $42(10 \mu \mathrm{s})$.

\begin{tabular}{|c|c|}
\hline Capture 1 & Capture 2 \\
\hline Span: 40 MHz & Span: $40 \mathrm{MHz}$ \\
\hline Resolution BW: 3.76 kHz & Resolution BW: 381.94 kHz \\
\hline Resolution BW Mode: Arbitrary & Resolution BW Mode: Arbitrary \\
\hline Resolution BW Couple: Fixed & Resolution BW Couple: Fixed \\
\hline Window Type: Flat Top (ampl acc) & Window Type: Flat Top (ampl acc) \\
\hline Frequency Points: 801 & Frequency Points: 801 \\
\hline Main Time Length: 1.015625 $\mu \mathrm{s}$ & Main Time Length: $10 \mu \mathrm{s}$ \\
\hline Max Overlap: 90\% (Av off) & Max Overlap: $90 \%$ (Av off) \\
\hline Max Overlap 0\% (Av On) & Max Overlap 0\% (Av On) \\
\hline Average Type: Off & Average Type: Off \\
\hline
\end{tabular}

The setup for the High-Power Interference Test is detailed in Figure 43.

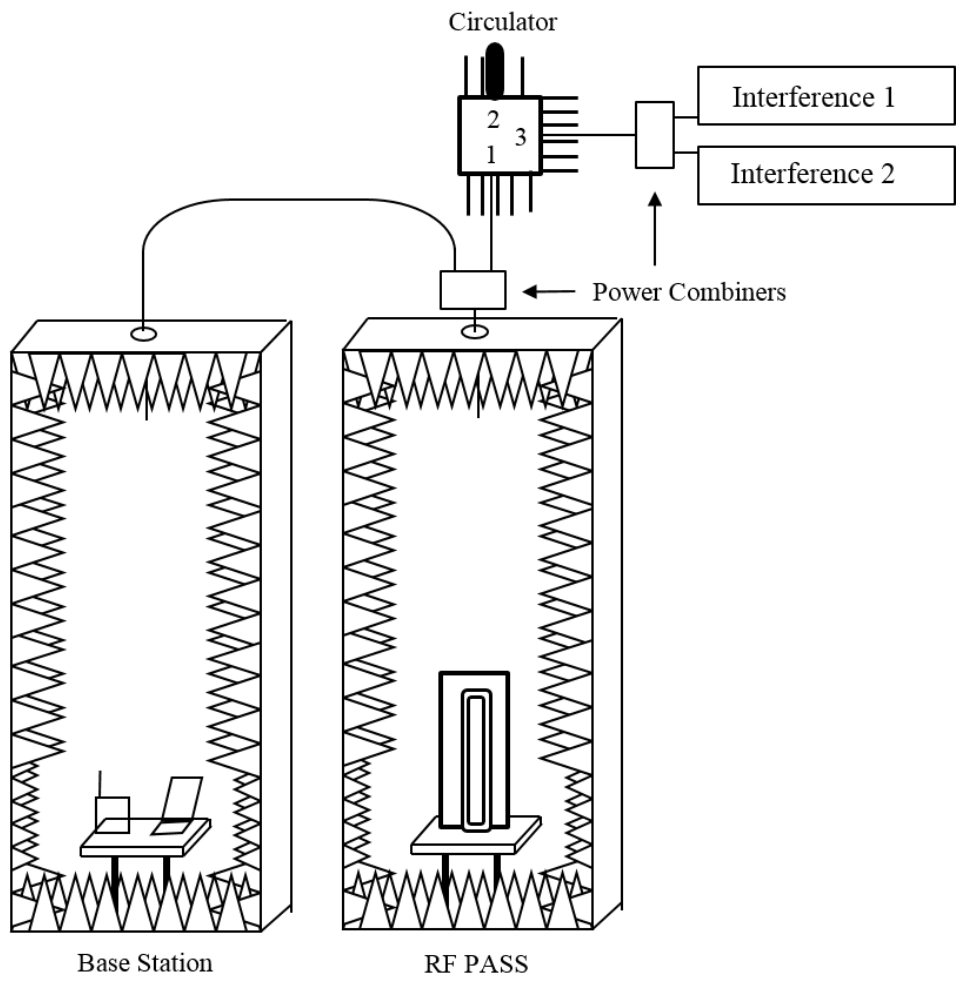

Figure 43. High-Power Interference Test setup.

Procedure

1) Turn RF PASS on, place on test platform, close all doors, keep pack in motion with dielectric rod.

2) Verify base station has received portable unit's "normal operation" signal (e.g., green icon), disconnect from base station chamber to ensure proper shielding, reconnect, and verify green. 
3) Introduce interference, wait 30 seconds.

4) Allow motion alarm to go off (stop moving pack).

5) Check that interference level is sufficiently high to disrupt the RF PASS system by verifying that no motion alarm is detected by the base station (wait 2 minutes).

6) Remove/turn off interference.

7) Count seconds until base station again registers motion alarm test.

Initially, results of this test showed that the interference did not disrupt the motion alarm. That is, the motion alarm continued to get through to the base station. We then inserted a broadband power amplifier into the setup and repeated the tests and verified that the motion alarm was disrupted as described in Step 5.

\subsubsection{Signal Generation}

\subsubsection{Background}

In this section, we describe in detail how to generate a wideband interference waveform suitable for testing wireless devices operating within the ISM bands. This work is in support of the NIST effort to establish a general framework for testing new wireless products used by the public-safety community. A necessary component of the tests should be an evaluation of how the wireless device responds and performs to external RF interference. Any device operating within the ISM bands may be subjected to a wide variety of channel interference scenarios. Of major concern is the possibility that the RF safety device has to operate under the condition of an elevated noise floor due to a uniformly-congested wireless channel. Thus, the motivation for developing the methodology presented in this technical note came from a need to subject the transceivers used by first responders to an interference signal which is persistent in both time and frequency across the entire ISM band.

\subsubsection{Theory}

A random process, or signal in our case, is classified as a white noise process if it has a constant power spectral density. The signal may be further categorized by its distribution; for example, a uniform distribution or a Gaussian distribution may both produce valid white noise processes. To generate a discrete white noise sequence, simply take independent random samples of a given distribution. If each sample is taken from the same distribution and all are mutually independent, this sequence is known as an independent and identically distributed (i.i.d.) random process. Another way of thinking about this sequence is as a realization of a vector of random variables which all have the same distribution. Furthermore, the mean and covariance of this sequence will not vary with respect to time which means this sequence is wide-sense stationary (WSS). In the frequency domain, the power spectral density function $S_{x x}(f)$ of the random process is given by the following equation:

$$
S_{X X}(f)=F\left[R_{X X}(\tau)\right]=\int_{-\infty}^{\infty} R_{X X}(\tau) e^{-j 2 \pi f \tau} d \tau,
$$

where $R_{x x}(\tau)$ is the auto-correlation function of the random process. Applying the WeinerKhintchine Theorem, we find that the power spectral density of the white noise process is constant 
across the entire frequency spectrum. The value of the constant will be equal to the variance of the random process:

$$
S_{X X}(f)=F\left[R_{X X}(\tau)\right]=\int_{-\infty}^{\infty} \sigma^{2} \delta(\tau) e^{-j 2 \pi f \tau} d \tau=\sigma^{2} \int_{-\infty}^{\infty} \delta(\tau) e^{-j 2 \pi f \tau} d \tau=\sigma^{2}
$$

Given that the power spectral density of a Gaussian white noise signal is constant for all frequencies, we may apply a digital filter to the white-noise signal to create an interference waveform of arbitrary spectral shape. The resulting complex IQ data are then uploaded to an arbitrary signal generator with the capability to up-convert and transmit IQ data.

\subsubsection{Method}

1. First we generate complex IQ vector as the sum of two independent zero-mean Gaussian distributions:

$$
Z=X+i Y
$$

where $\mathrm{X}$ and $\mathrm{Y}$ are random vectors generated with the MATLAB command randn. The associated covariance matrix of $X$ and $Y$ should be very close to the ideal $\left[\begin{array}{ll}1 & 0 \\ 0 & 1\end{array}\right]$. Figure 44 through Figure 47 show various forms of the $100 \mathrm{MHz}$ wide interference signal.

$\mathrm{L}=1 * 10 \wedge 6$; $\quad$ \% Define Length of IQ Vector

IQ_complex $=\operatorname{randn}(L, 1)+1 j * \operatorname{randn}(L, 1)$;

2. Define the parameters of the arbitrary signal generator and the desired interference waveform attributes.

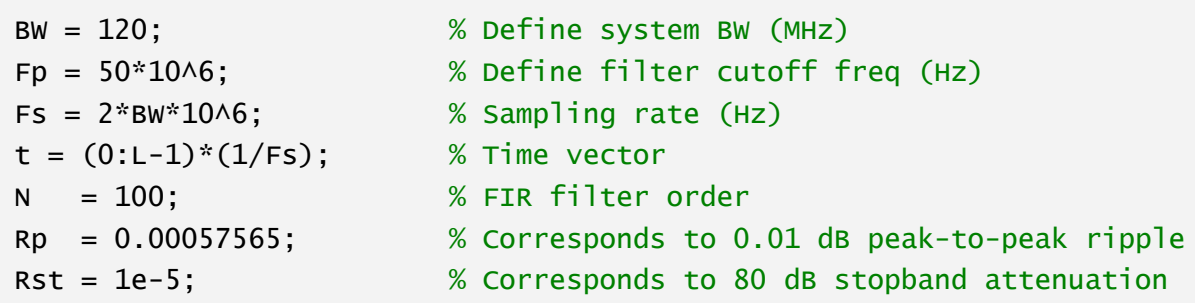

3. Generate and apply digital filter to IQ vector. There is a lot of freedom in choosing which filter to apply but, for our purposes, we chose a constrained equiripple finite impulse response (FIR) filter which may be designed with the MATLAB command firceqrip.

NUM = firceqrip $(\mathrm{N}, \mathrm{Fp} /(\mathrm{Fs} / 2)$, [Rp Rst], 'passedge' $) ; \%$ NUM = vector of coeffs

IQ_time_filt $=$ filter(NUM,1, IQ_complex);

4. Save filtered IQ data in file format appropriate for arbitrary signal generator. 
IQ_save $=$ [real(IQ_time_fi1t), imag(IQ_time_fi1t)];

d7mwrite('noiselong1.txt' , IQ_save, 'delimiter' , ' , ', 'newline' , 'pc');

\subsubsection{Plots}

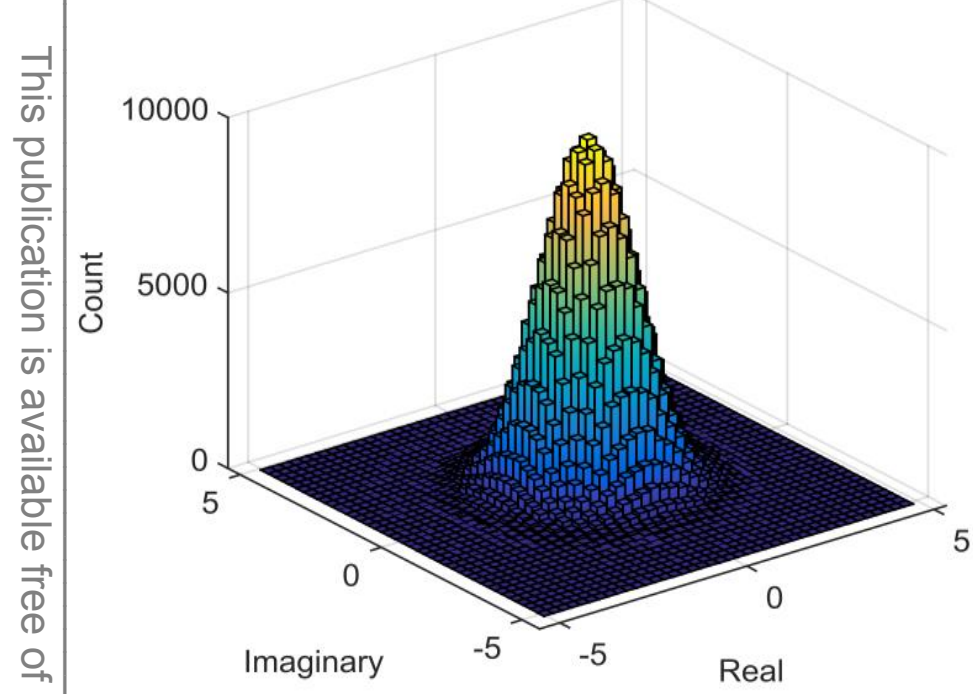

Figure 44: Bivariate histogram of Gaussian white noise data corresponding to a vector of length $\boldsymbol{L}=\mathbf{1 0}^{\mathbf{6}}$.

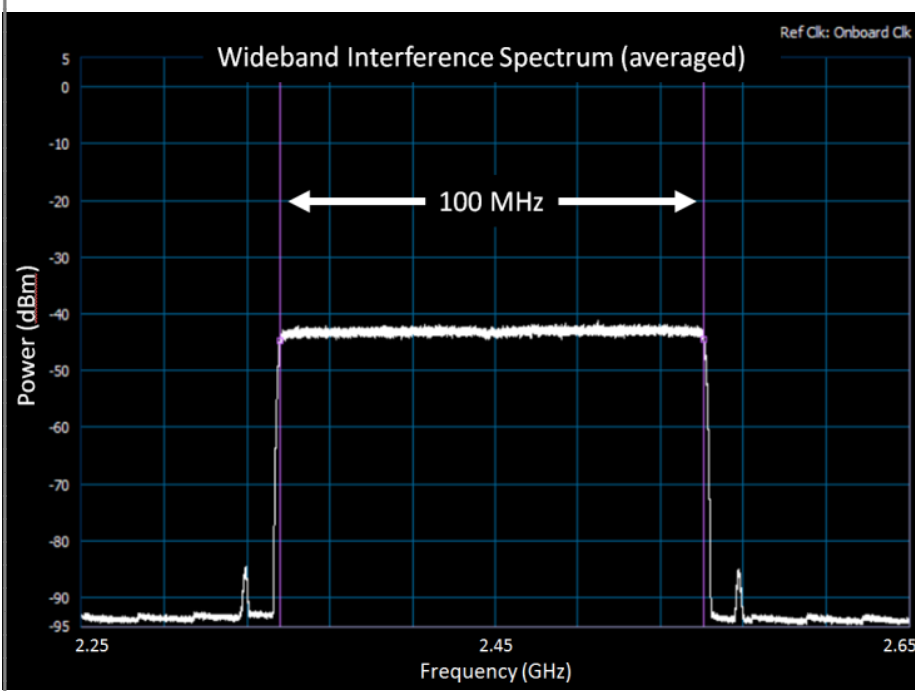

Figure 46: Measured spectrum of $100 \mathrm{MHz}$ wide interference waveform.
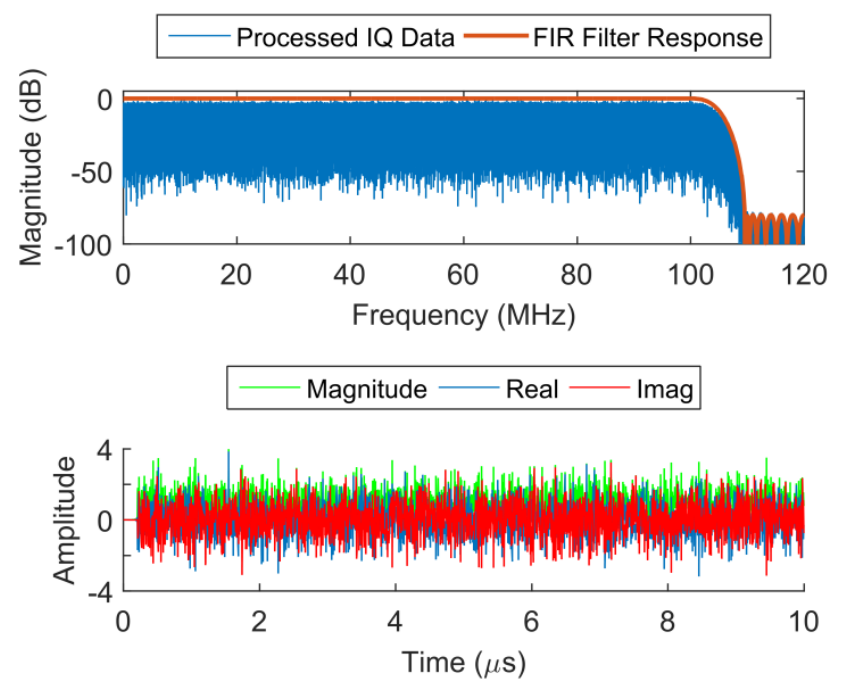

Figure 45: Realization of $100 \mathrm{MHz}$ wide interference waveform.

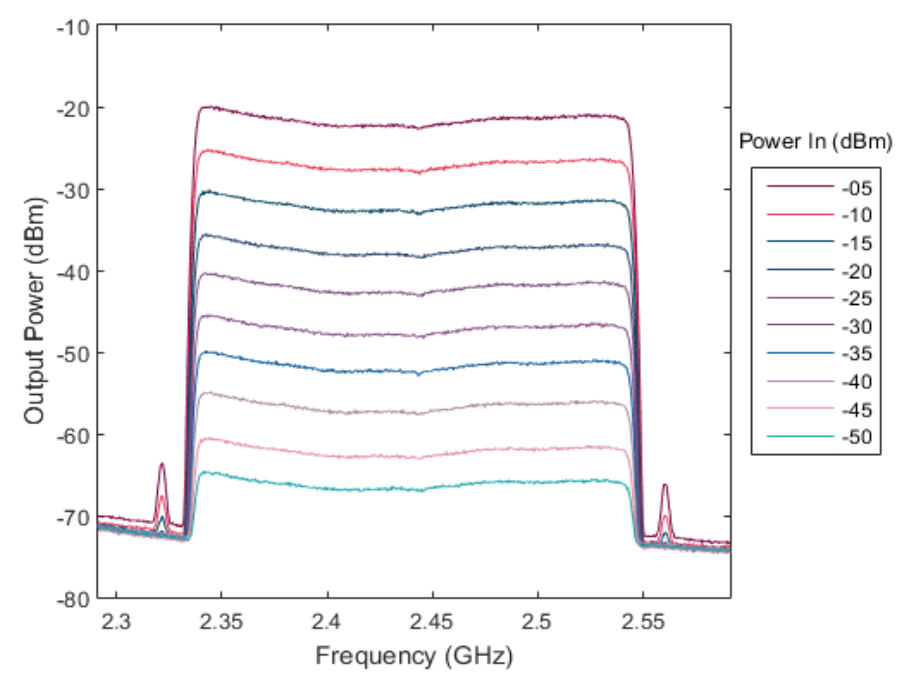

Figure 47: Magnitude response of a power amplifier to a $200 \mathrm{MHz}$ wide interference signal. 


\subsubsection{NIST Results}

\subsubsection{Manufacturer 3, High-Power Interference Test Results}

Tests were performed at NIST and results can be seen in Table 26 through Table 28.

Table 26. Manufacturer 3, High-Power Interference Test results for Pack 1.

\begin{tabular}{|c|c|}
\hline \multicolumn{2}{|c|}{ Pack 1 } \\
\hline Trial Number & Response Time (sec) \\
\hline 1 & 27.58 \\
\hline 2 & 28.56 \\
\hline 3 & 22.83 \\
\hline 4 & 26.46 \\
\hline 5 & 16.10 \\
\hline 6 & 13.51 \\
\hline 7 & 11.98 \\
\hline 8 & 7.15 \\
\hline 9 & 21.00 \\
\hline 10 & 36.03 \\
\hline
\end{tabular}

Table 27. Manufacturer 3, High-Power Interference Test results for Pack 2.

\begin{tabular}{|c|c|}
\hline \multicolumn{2}{|c|}{ Pack 2 } \\
\hline Trial Number & Response Time $(\mathrm{sec})$ \\
\hline 1 & 35.58 \\
\hline 2 & 26.05 \\
\hline 3 & 28.79 \\
\hline 4 & 15.53 \\
\hline 5 & 29.41 \\
\hline 6 & 19.00 \\
\hline 7 & 29.75 \\
\hline 8 & 30.08 \\
\hline 9 & 12.05 \\
\hline 10 & 30.93 \\
\hline
\end{tabular}

Table 28. Manufacturer 3, High-Power Interference Test results for Pack 3.

\begin{tabular}{|c|c|}
\hline \multicolumn{2}{|c|}{ Pack 3 } \\
\hline Trial Number & Response Time $(\mathrm{sec})$ \\
\hline 1 & 12.49 \\
\hline 2 & 20.71 \\
\hline 3 & 8.31 \\
\hline 4 & 31.16 \\
\hline 5 & $180+$ \\
\hline 6 & 14.93 \\
\hline 7 & 19.60 \\
\hline 8 & 17.73 \\
\hline 9 & 33.00 \\
\hline 10 & 16.78 \\
\hline
\end{tabular}




\subsubsection{Manufacturer 1, High-Power Interference Test Results}

Tests were performed at NIST. Settings are shown in Table 29 and results can be seen in Table 30 through Table 32 and Figure 48.

Table 29. Manufacturer 1 High-Power Interference Test settings.

\begin{tabular}{|c|c|}
\hline Center Frequency of Interference 1 & $863.5 \mathrm{MHz}$ \\
\hline Center Frequency of Interference 2 & $966.5 \mathrm{MHz}$ \\
\hline Level & $-20 \mathrm{dBm}$ \\
\hline Amplifier & $50 \%$ Gain \\
\hline
\end{tabular}

Table 30. Manufacturer 1, High-Power Interference Test results for Pack 1.

\begin{tabular}{|c|c|}
\hline \multicolumn{2}{|c|}{ Pack 1 } \\
\hline Trial Number & Response Time $(\mathrm{sec})$ \\
\hline 1 & 5.88 \\
\hline 2 & 6.45 \\
\hline 3 & 9.23 \\
\hline 4 & 5.40 \\
\hline 5 & 4.60 \\
\hline 6 & 7.38 \\
\hline 7 & 6.25 \\
\hline 8 & 5.96 \\
\hline 9 & 6.28 \\
\hline 10 & 3.99 \\
\hline
\end{tabular}

Table 31. Manufacturer 1, High-Power Interference Test results for Pack 2.

\begin{tabular}{|c|c|}
\hline \multicolumn{2}{|c|}{ Pack 2 } \\
\hline Trial Number & Response Time (sec) \\
\hline 1 & 7.60 \\
\hline 2 & 5.83 \\
\hline 3 & 8.81 \\
\hline 4 & 6.16 \\
\hline 5 & 4.45 \\
\hline 6 & 8.56 \\
\hline 7 & 6.91 \\
\hline 8 & 7.08 \\
\hline 9 & 10.48 \\
\hline 10 & 8.75 \\
\hline
\end{tabular}


Table 32. Manufacturer 1, High-Power Interference Test results for Pack 3.

\begin{tabular}{|c|c|}
\hline \multicolumn{2}{|c|}{ Pack 3 } \\
\hline Trial Number & Response Time $(\mathrm{sec})$ \\
\hline 1 & 5.71 \\
\hline 2 & 7.85 \\
\hline 3 & 7.71 \\
\hline 4 & 5.84 \\
\hline 5 & 8.61 \\
\hline 6 & 5.78 \\
\hline 7 & 5.78 \\
\hline 8 & 7.55 \\
\hline 9 & 7.75 \\
\hline 10 & 11.03 \\
\hline
\end{tabular}
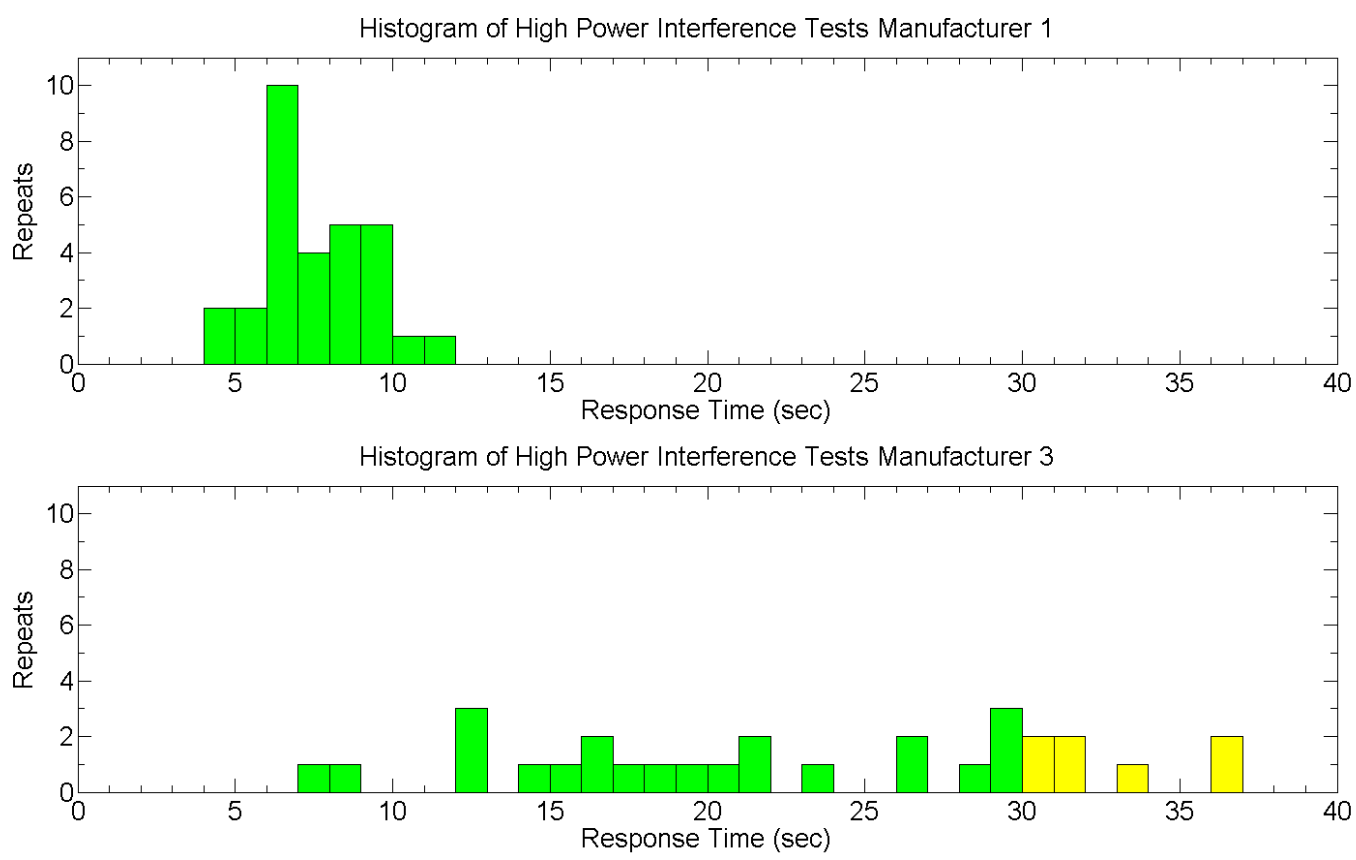

Figure 48. Histogram of High-Power Interference Tests for Manufacturers 1 and 3. The 180+ trial is not shown in the histogram (as seen in the table).

\section{Conclusion}

We described the development of test methods designed to aid standards bodies with the evaluation of wireless technology used in firefighter, public-safety, and other applications where communications are utilized. The test methods described here were designed to be as costeffective as possible so that, not only test laboratories, but manufacturers and even end users can reproduce them for design, test, and evaluation purposes. 
We anticipate that, as more and more wireless electronic-safety equipment becomes available, the test methods described here will be used for testing those systems as well. 


\section{References}

[1] National Fire Protection Association, "NFPA® 1982 Standard on Personal Alert Safety Systems (PASS), 2013 Edition," 2013.

[2] K.A. Remley and W. F. Young, "Test methods for RF-based electronic safety equipment: Part 1 - From field tests to performance metrics," IEEE Electromagnetic Compatibility Magazine, vol.1, no.4, pp.53-60, Fourth Quarter 2012.

[3] K.A. Remley and W. F. Young, "Test methods for RF-based electronic safety equipment: Part 2 - Development of laboratory-based tests," IEEE Electromagnetic Compatibility Magazine, vol.2, no.1, pp.70-80, 1St Quarter 2013.

[4] K.A. Remley, W.F. Young, and J. Healy, "Analysis of radio-propagation environments to support standards development for RF-based electronic safety equipment," NIST Technical Note 1559, 2012.

[5] K. A. Remley, G. Koepke, C. L. Holloway, C. Grosvenor, D. Camell, J. Ladbury, R. T. Johnk, D. Novotny, W. F. Young, G. Hough, M. D. McKinley, Y. Becquet, and J.Korsnes, "Measurements to Support Modulated-Signal Radio Transmissions for the Public-Safety Sector," Natl. Inst. of Stand. Technol. Note 1546, June 2008.

[6] Joint Task Force IEC SC77B-CISPR/A, Electromagnetic Compatibility (EMC)—Part 4.21: Testing and Measurement Techniques-Reverberation Chamber Test Methods, IEC 61000-4-21, Geneva, Switzerland, Aug. 2003.

[7] B. N. Taylor, C. E. Kuyatt, "Guidelines for Evaluating and Expressing the Uncertainty of NIST Measurement Results,” NIST Technical Note 1297, September, 1994.

[8] D. Hill, "Electromagnetic Fields in Cavities: Deterministic and Statistical Theories," IEEE Press, Copyright 2009.

[9] P. Besnier and B. Démoulin, "Electromagnetic Reverberation Chambers," ISTE Ltd, Copyright 2011.

[10] H.G. Krauthäuser, "On the measurement of total radiated power in uncalibrated reverberation chambers," IEEE Trans. Electromagnetic Compat., vol. 49, no. 2, May 2007, pp. 270-279.

[11] K. Banerjee, M. van Doorn, and P. Beeckman, "Using the Reverberation Chamber to Measure Total Radiated Power for EMF Assessments," Interference Technology, Nov. 2013.

[12] X. Chen, P.-S. Kildal, C. Orlenius, J. Carlsson, "Channel sounding of loaded reverberation chamber for over-the-air testing of wireless devices - coherence bandwidth versus average mode bandwidth and delay spread," IEEE Ant. Wireless Propagat. Lett., vol. 8, 2009, pp. 678-681.

[13] K. A. Remley, C.-M. Wang, D. F. Williams, J. J. aan den Toorn, C. L. Holloway “A Significance Test for Reverberation-Chamber Measurement Uncertainty in Total Radiated Power of Wireless Devices," IEEE Trans. Electromagnetic Compat., vol 58, no. 1, Feb 2016, pp. 207-219. 
[14] K. A. Remley, G. Koepke, C. Grosvenor, R. T. Johnk, J. Ladbury, D. Camell, and J. Coder, "NIST Tests of the Wireless Environment in Automobile Manufacturing Facilities," Natl. Inst. of Stand. Technol. Note 1550, Oct. 2008.

[15] K. A. Remley, G. Koepke, C. L. Holloway, C. Grosvenor, D. Camell, J. Ladbury, R. T. Johnk, D. Novotny, W. F. Young, G. Hough, M. D. McKinley, Y. Becquet, and J. Korsnes, "Measurements to Support Modulated-Signal Radio Transmissions for the Public-Safety Sector," Natl. Inst. of Stand. Technol. Note 1546, June 2008.

[16] J. M. Ladbury and K. Goldsmith, "Reverberation Chamber Verification Procedures, or, How to Check if Your Chamber Ain't Broke and Suggestions on How to Fix It if It Is," IEEE International Symposium on Electromagnetic Compatibility, 2000, vol.1, no., pp.1722, 2000.

[17] K. A. Remley, S. J. Floris, H. A. Shah, and C. L. Holloway, "Static and Dynamic Propagation-Channel Impairments in Reverberation Chamber," IEEE Trans. Electromagnetic Compat., vol. 53, no. 3, pp 589-599, Aug, 2011.

[18] W. F. Young, C. L. Holloway, G. Koepke, D. Camell, Y. Becquet, K. A. Remley, "RadioWave Propagation Into Large Building Structures-Part1: CW Signal Attenuation and Variability" IEEE Trans. Antennas and Propagat, vol. 58, no.4, pp. 1290-1301, April, 2010.

[19] "American National Standard Recommended Practice for an On-Site, Ad Hoc Test Method for Estimating Radiated Electromagnetic Immunity of Medical Devices to Specific RadioFrequency Transmitters," ANSI C63.18-1997, Piscataway, NJ: IEEE, 1997.

[20] N. J. LaSorte, H. H. Refai, D. M. Witters Jr., S. J. Seidman, J. L. Silberberg,"Wireless medical device coexistence," Medical Electronics Design, Aug. 2011.

[21] National Institute for Occupational Safety and Health, "Fire fighter fatality investigation and prevention program," http://www.cdc.gov/niosh/fire/, accessed Jan. 2012.

[22] T. Keller, J. Modelski, "Experimental results of testing interferences in $2.4 \mathrm{GHz}$ ISM band," 33rd European Microwave Conference, pp. 1043-1046, Oct. 2003.

[23] S. Seidman, W. Kainz, P. Ruggera, and G. Mendoza, "Wireless coexistence and EMC of Bluetooth and $802.11 \mathrm{~b}$ devices in controlled laboratory settings," Open Biomed. Eng. J.," 2011, vol. 5, pp. 74-82.

[24] http://transition.fee.gov/oet/info/rules

[25] ftp://ftp10.dlink.com/pdfs/products/DAP-2553/DAP-2553_ds.pdf 